\title{
WestVirginiaUniversity
}

THE RESEARCH REPOSITORY @ WVU

Graduate Theses, Dissertations, and Problem Reports

2021

\section{Manipulating the Perineuronal Net in the Deep Cerebellar Nucleus}

Deidre E. O'Dell

deodell@mix.wvu.edu

Follow this and additional works at: https://researchrepository.wvu.edu/etd

Part of the Behavioral Neurobiology Commons, Laboratory and Basic Science Research Commons, and the Other Chemicals and Drugs Commons

\section{Recommended Citation}

O'Dell, Deidre E., "Manipulating the Perineuronal Net in the Deep Cerebellar Nucleus" (2021). Graduate Theses, Dissertations, and Problem Reports. 8096.

https://researchrepository.wvu.edu/etd/8096

This Dissertation is protected by copyright and/or related rights. It has been brought to you by the The Research Repository @ WVU with permission from the rights-holder(s). You are free to use this Dissertation in any way that is permitted by the copyright and related rights legislation that applies to your use. For other uses you must obtain permission from the rights-holder(s) directly, unless additional rights are indicated by a Creative Commons license in the record and/ or on the work itself. This Dissertation has been accepted for inclusion in WVU Graduate Theses, Dissertations, and Problem Reports collection by an authorized administrator of The Research Repository @ WVU.

For more information, please contact researchrepository@mail.wvu.edu. 
Graduate Theses, Dissertations, and Problem Reports

2021

Manipulating the Perineuronal Net in the Deep Cerebellar Nucleus

Deidre E. O'Dell

Follow this and additional works at: https://researchrepository.wvu.edu/etd

Part of the Behavioral Neurobiology Commons, Laboratory and Basic Science Research Commons, and the Other Chemicals and Drugs Commons 


\title{
Manipulating the Perineuronal Net in the Deep Cerebellar Nucleus
}

\section{Deidre E. O'Dell}

\author{
Dissertation \\ Submitted to School of Medicine \\ at West Virginia University \\ in partial fulfillment of the requirements for the degree of \\ Doctor of Philosophy \\ In Neuroscience
}

\author{
Bernard G. Schreurs, Ph.D., chair \\ James Simpkins, Ph.D., co-chair \\ Albert Berrebi, Ph.D. \\ Adrienne Salm, Ph.D. \\ Eric Tucker, Ph.D. \\ Scott Weed, Ph.D. \\ Departments of Cancer Cell Biology and Neuroscience
}

Morgantown, West Virginia

2021

Keywords: deep cerebellar nuclei, eyeblink conditioning, learning, memory, perineuronal nets

Copyright 2021 Deidre E. O’Dell 


\begin{abstract}
Manipulating Manipulating the Pthe Perineurerineuronal Net in onal Net in the Deep Certhe Deep Cerebellar Nucleusebellar Nucleus
\end{abstract}

\title{
Deidre E. O'Dell
}

Perineuronal nets (PNN) are a type of specialized extracellular matrix in the central nervous system. The PNN forms during postnatal development but the ontogeny of the PNN has yet to be elucidated. Studying the PNN in the rat brain may allow us to further understand the PNN's role in development, learning, and memory. The PNN is fully developed in the deep cerebellar nuclei (DCN) of rats by post-natal day 18. By using enzymatic digestion of the PNN with chondroitinase ABC (ChABC), we studied how digestion of the PNN affects cerebellar-dependent eyeblink conditioning (EBC) and performed electrophysiological recordings from DCN neurons. In vivo degradation of the PNN resulted in differences in EBC amplitude and area. Female animals in the vehicle group demonstrated higher levels of conditioning as well as higher post-probe conditioned responses compared to males in that group, differences not present in the ChABC group. In vitro, DCN neurons with disrupted PNNs following exposure to ChABC had altered membrane properties, fewer rebound spikes, and decreased intrinsic excitability. Doxycycline, an antibiotic, can inhibit endogenous enzymes that digest the PNN. Rats given doxycycline had higher PNN staining in the DCN compared to vehicle. Animals receiving doxycycline prior to behavior have a smaller eyeblink area in comparison to the vehicle group. However, these rats also had more unconditioned responses, suggesting in addition to preventing the PNN from being remodeled, doxycycline may cause nonassociative effects. This study further elucidates the role of the PNN in cerebellar learning. 


\section{Acknowledgements}

I would like to thank my advisor, Dr. Bernard G. Schreurs. Through his wonderful mentorship I was able to complete what seemed like an insurmountable task sometimes. I appreciate his enthusiasm, patience, and support throughout my journey to finishing my PhD. I am truly grateful for everything.

I also must offer gratitude to my committee members, Dr. Albert Berrebi, Dr. Adrienne Salm, Dr. James Simpkins, Dr. Eric Tucker, and Dr. Scott Weed. Their guidance was invaluable to making this project a success.

The past and present members of Schreurs lab have also been invaluable assets on this journey. Past members, Dr. Lauren Burhans and Dr. Sylwia Brooks provided me with training that was essential to completing this work. Dr. Desheng Wang has been my constant surgical assistant the past few years and his electrophysiological expertise was truly a great benefit to completing this project. Maybe I will finally be able to take his advice and relax a little! Carrie Smith-Bell and Roger Bell have taught me a great deal and always assisted me in every way possible. All of you have made this time much easier and enjoyable for me. I hope each of you know how glad I am to have worked with you and how greatly I appreciate what you have taught me. Thank you, also, to the myriad students who briefly passed through the lab for your help.

To the OLAR, ACUC, vet, and husbandry staff, thank you for all of your assistance. From helping me push surgical tables through the halls and caring for my animals, you are all greatly appreciated. To my rats, thanks for teaching me not to judge what society deems gross or scary and for your sacrifice.

To the WVU Neuroscience Department, thank you for providing me with this education and experience and striving to provide the best support to your students.

Lastly, to my dear friends and family, I would have failed a thousand times without you. To my friends "in the trenches" currently or previously at WVU, thanks for holding me up, making me laugh, for the study times, practice talks, and sending me papers related to my work (the science love language). To Catherine, Rachel, Skye, and Tiffany, I am blessed to know such brilliant, kind, and talented women of science. I am in awe of each of you, as scientists and as people. To Catherine and Skye, thanks for befriending this weirdo and sticking by me through all of my ups and downs. To my friend's outside of the programs, thank you for listening to my seemingly bizarre rants and never failing in your support. Nick and Gary, you are two of the best guys I know and thanks for keeping my spirits up. To Regi, from memes of the Greek pantheon to kind words of encouragement, you are gem, hrrnnngngngh! To Jess, my bestie, I cannot express the gratitude for your help and wisdom when I would feel lost. To my wonderful parents, thank you for never doubting me and being my biggest cheerleaders. Thank you, and Bubby, for your love and incessant prayer on my behalf. To my wonderful in-laws, thank you for not running for the hills when you truly got to know who married into your family and for your love! To the rest of my family, thank you for supporting me! To my Sean, you are my rock. Thank you for putting up with bats, flooded basements, and leaking roofs all so your wife can pursue her dream of getting a PhD. You are the most important person, and this would have never happened without you in my corner. I love you. 


\section{Table of Contents}

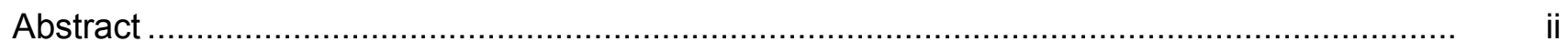

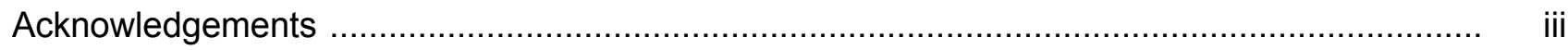

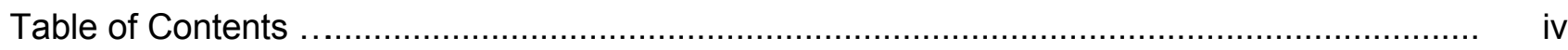

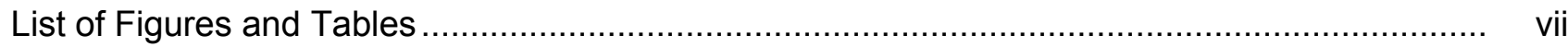

Chapter 1. Introduction to the Perineuronal Net............................................... 1

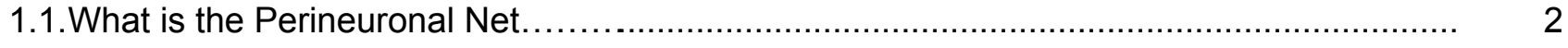

1.2.PNN and Learning and Memory ........................................................... 6

1.2.1. The PNN is altered in diseases affecting Learning and Memory............................ 6

1.2.2. PNN Manipulations and Learning and Memory.......................................... 11

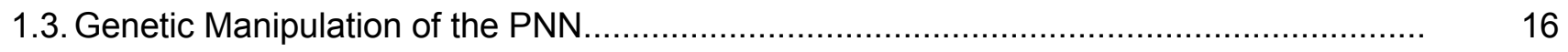

1.4. Functional connections and activity are required for normal PNN development................ 18

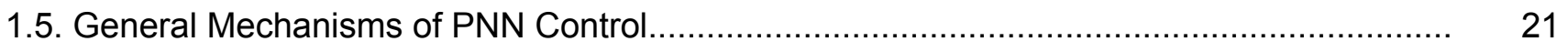

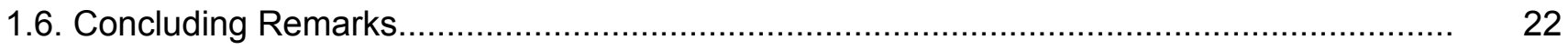

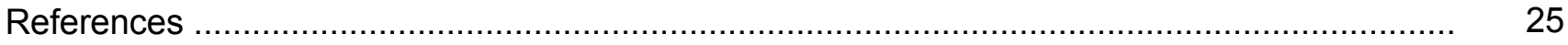

Chapter 2. The Perineuronal net and the Cerebellum........................................... 35

2.1. The Perineuronal Net is abundant in the cerebellum................................................ 36

2.2. The Cerebellum is an archetypal system fit for many studies related to the PNN............... 37

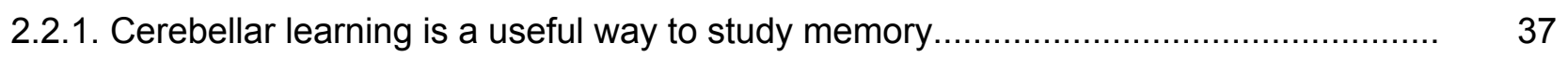

2.2.2. The cerebellum is connected to other brain regions that are also $\mathrm{PNN}+\ldots \ldots \ldots \ldots \ldots \ldots . . . \ldots$

2.2.3. Development in the cerebellum alters behavior and may be related to the PNN........ 40

2.3. Manipulating the PNN in the Cerebellum will alter behavior........................................... 42

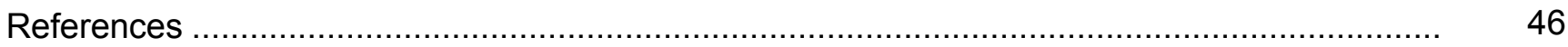

Chapter 3. Disruption of Rat Deep Cerebellar Perineuronal Net Alters Eyeblink Conditioning and Neuronal Physiology (O'Dell et al, Neurobiology of Learning

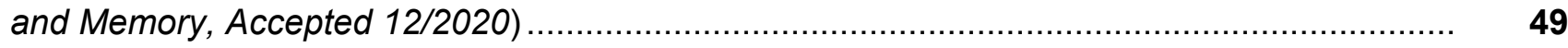

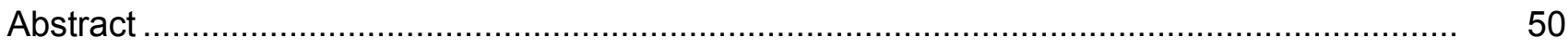

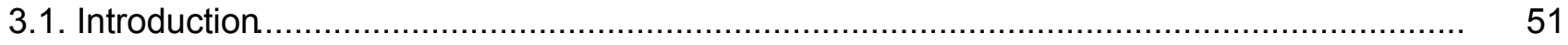

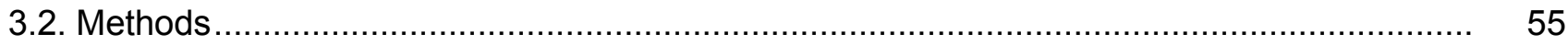

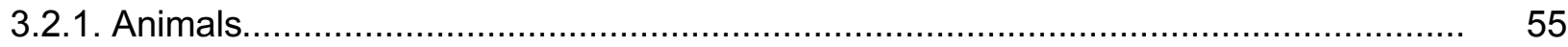




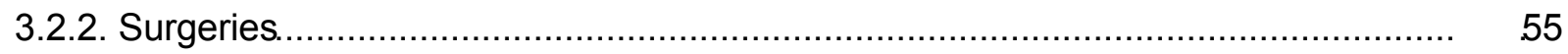

3.2.3. Eyeblink Conditioning........................................................................... 56

3.2.4. Behavioral Data Analysis........................................................................ 58

3.2.5.Slice preparation and patch-clamp recordings:.................................................. $\quad 59$

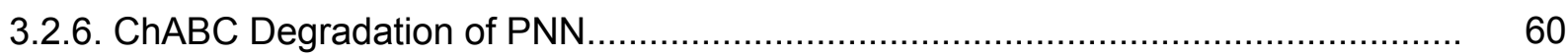

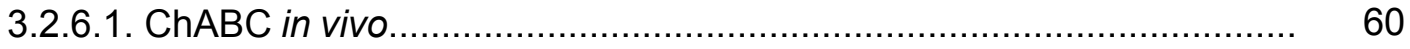

3.2.6.2. ChABC in vitro incubation............................................................ 61

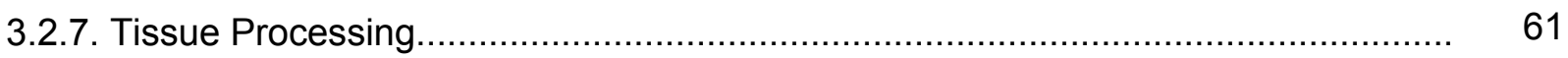

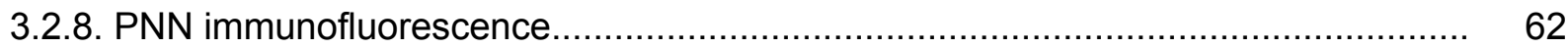

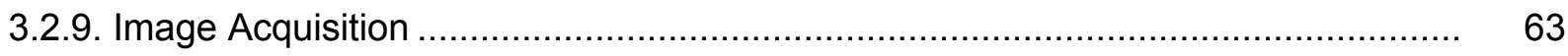

3.2.10. Statistical Analysis ................................................................................. 64

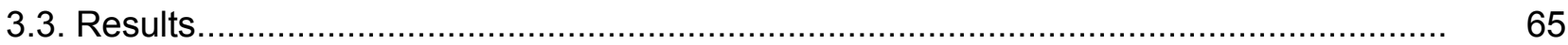

3.3.1. Ontogenetic Differences in the AIN............................................................. 65

3.3.2. PNN Digestion In Vivo alters Eyeblink Conditioning.......................................... 65

3.3.2.1. In Vivo PNN Digestion................................................................ 65

3.3.2.2. EBC Acquisition.................................................................. 68

3.3.2.3. EBC Extinction..................................................................... $\quad 70$

3.3.2.4. Sex Differences........................................................................ $\quad 70$

3.3.3. In Vitro PNN Digestion Alters the Electrophysiological Properties of Neurons

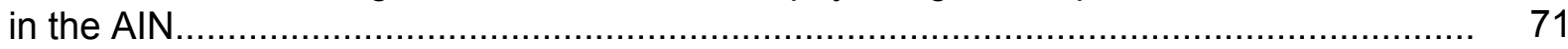

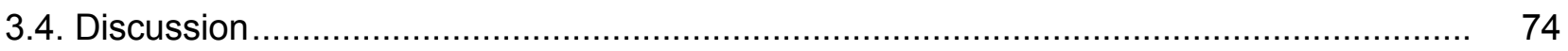

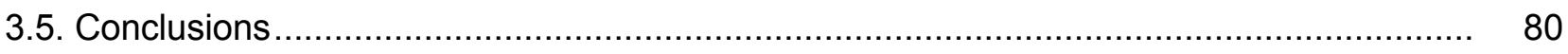

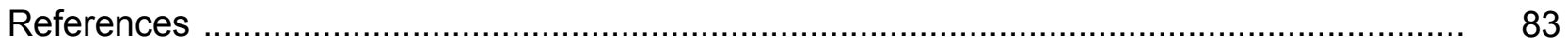

Chapter 4. Doxycycline Alters Eyeblink Conditioning and the Perineuronal Net in Rat Deep Cerebellar Nuclei .............................................................................. 92

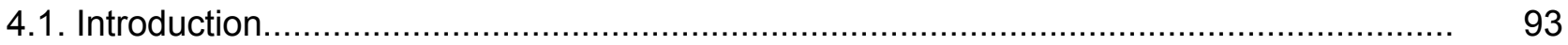

4.2. Methods ................................................................................................... 95

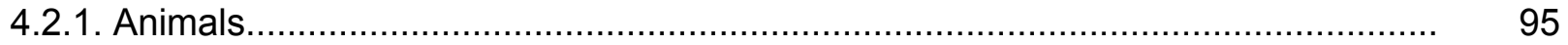

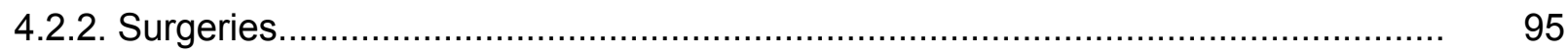

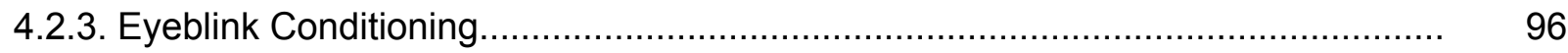

4.2.4. Behavioral Data Analysis.......................................................................... 97 
4.2.5. Doxycycline mediated manipulation of the PNN ........................................ 98

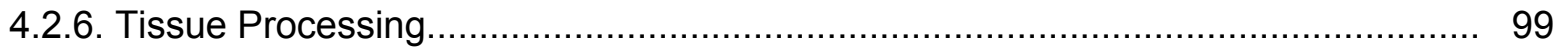

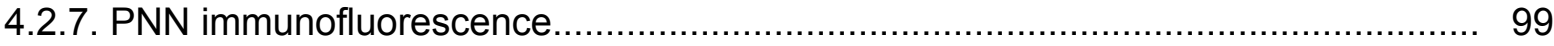

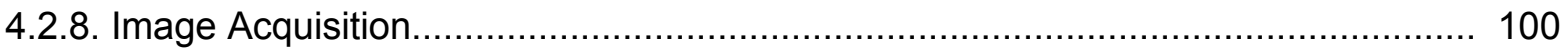

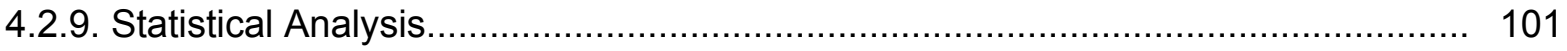

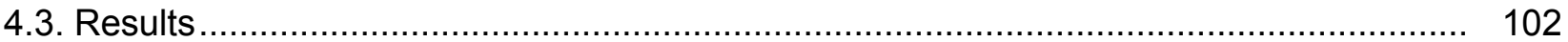

4.3.1. Doxycycline may prevent modulation of the PNN in the AIN ............................. 102

4.3.1.1. Acute Doxycycline Injections........................................... 102

4.3.1.2. Daily Doxycycline Injections prior to EBC.................................. 102

4.3.2. Doxycycline does not alter Eyeblink Conditioning......................................... 102

4.3.2.1. EBC Acquisition.................................................................. 102

4.3.2.2. EBC Extinction.................................................................. 104

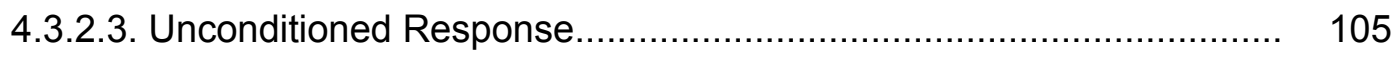

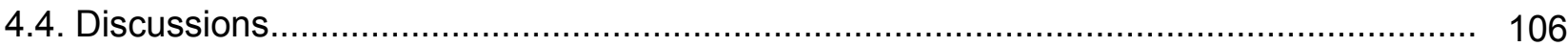

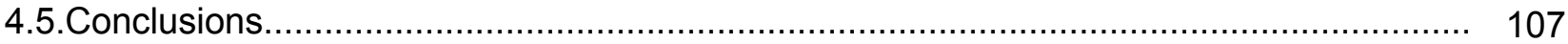

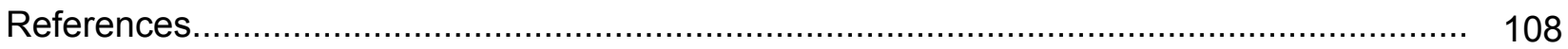

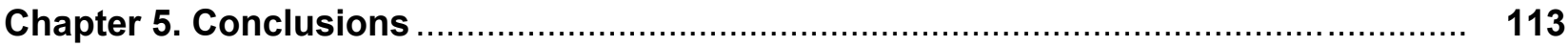

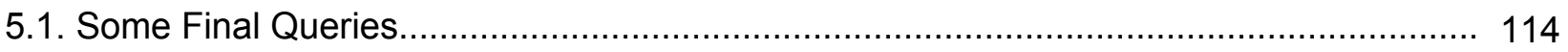

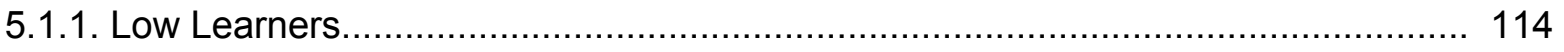

5.1.2. Low Learners and ChABC digestion Level................................................. 116

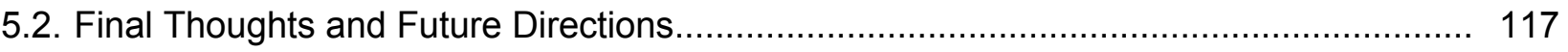

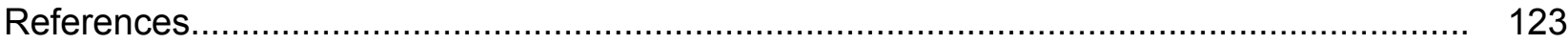




\section{List of Figures and Tables}

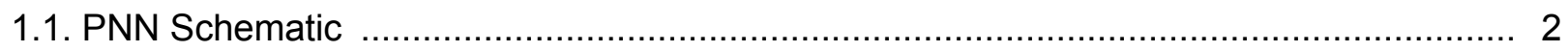

1.2. PNN, plasticity, development, and manipulation....................................................... 5

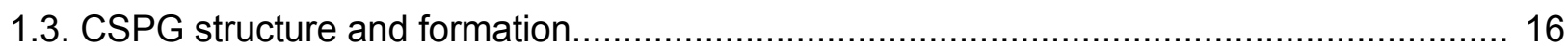

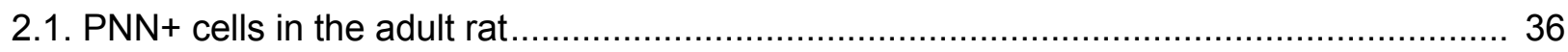

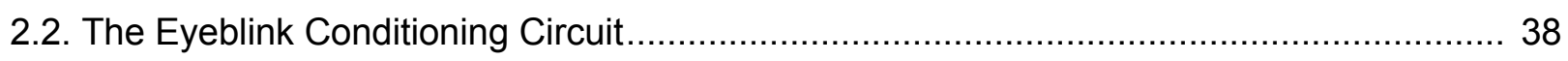

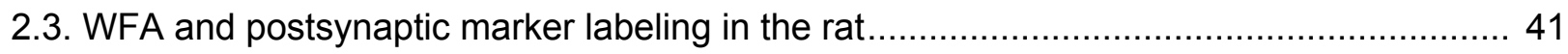

2.4. EBC acquisition and extinction may alter PNN+ cells of the $\mathrm{DCN}$............................... 44

Table 3.1. Materials used in Tissue Processing................................................................ 62

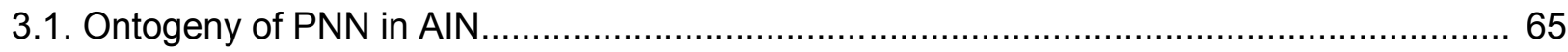

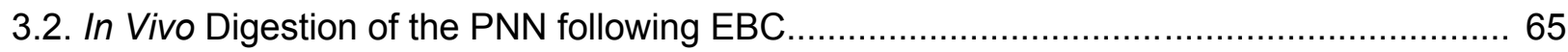

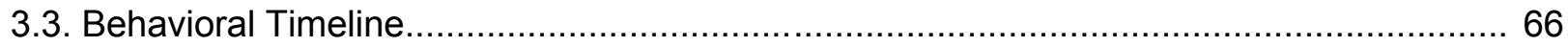

3.4. Percent Conditioned Responses between Vehicle and ChABC rats during

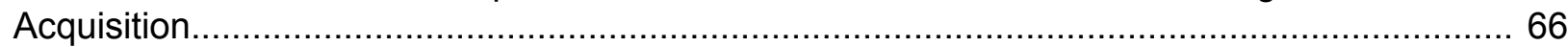

3.5. Amplitude of the Conditioned Response between ChABC and Vehicle Rats..................... 67

3.6. Area of the Conditioned Response between ChABC and Vehicle Rats............................ 68

3.7. \%CR Sex Differences between ChABC and Vehicle Rats during Acquisition..................... 69

3.8. Area between ChABC and Vehicle Rats during Acquisition......................................... 70

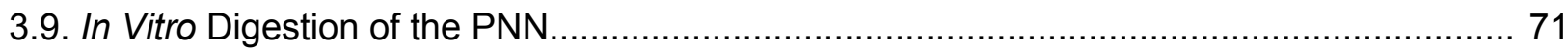

Table 3.2. ChABC treatment altered membrane properties of rat DCN neurons..................... 71

Table 3.3. ChABC treatment affected the properties of rebound spikes from

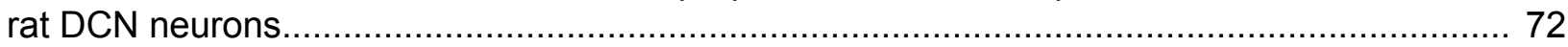

3.10. ChABC altered the electrophysiological properties of AIN cells................................... 74

3.11. ChABC exposure altered the electrophysiological properties of AIN cells....................... 75

3.12. ChABC exposure changed the properties of hyperpolarization induced

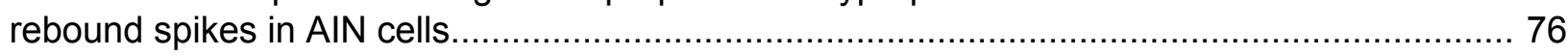

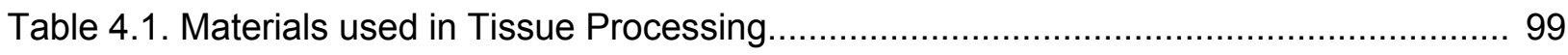

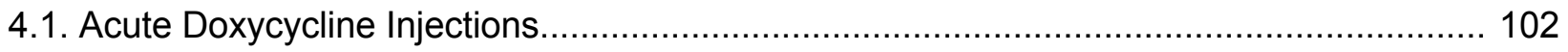

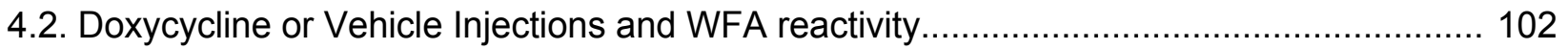

4.3. Percent Conditioned Responses between Doxycycline and Vehicle Rats........................ 103

4.4. Peak Latency of Conditioned Responses between Doxycycline and

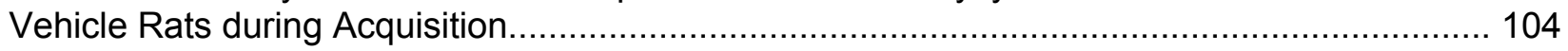

4.5. Area of Conditioned Responses between Doxycycline and Vehicle Rats......................... 105 
4.6. Unconditioned Responses between Doxycycline and Vehicle Rats........................... 106

5.1. Percent Conditioned Responses between low learning ChABC and Vehicle Rats.......... 114

5.2. Amplitude and Area of the Conditioned Response between low learning

ChABC and Vehicle Rats.

5.3. Eyeblink Latency of the Conditioned Response between low learning

ChABC and Vehicle Rats.

5.4. Amplitude of the Conditioned Response between low learning

ChABC and Vehicle Rats.

5.5. Area of the Conditioned Response between low learning ChABC and Vehicle Rats. 
Chapter 1. Introduction to the Perineuronal Net 


\subsection{What is the Perineuronal Net?}

The perineuronal net (PNN) was originally described by the notable neuroscientists Golgi and Ramon y Cajal in the 1890s (Celio et al., 1998) but has recently reemerged as an exciting target of study in many areas of neuroscience. Studying the PNN is important because there is strong evidence that it exerts control over plasticity, learning, and memory in both healthy and disease states. Many studies have attempted to fully understand how the PNN influences these properties. From fear conditioning to Alzheimer's disease, researchers continue to find roles for the PNN in the central nervous system. The resurgence in interest provides critical new insights into the function of the

PNN more than 100 years after its discovery. The results suggest novel approaches to treating neuronal disorders and will drive additional research to define the mechanistic basis for regulating learning and memory by the PNN.

The PNN is a specialized type of extracellular matrix that is deposited onto groups of fast- spiking interneurons and large excitatory deep cerebellar neurons in the central
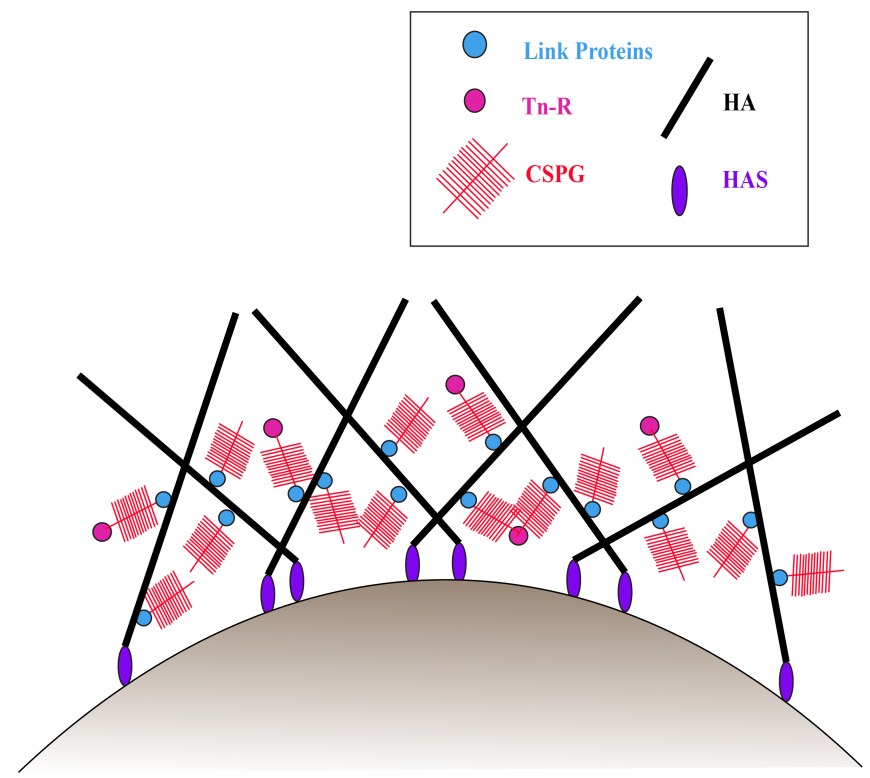

Figure 1.1. PNN Schematic.

This schematic shows the various elements that comprise the PNN. HAS has been implicated as the anchor of the PNN and secretes $\mathrm{HA}$ at the cell surface. Link proteins join CSPG to HA. Tn-R can bind up to three CSPG to further reinforce the structure.

CSPG: Chondroitin Sulfate Proteoglycans, HAS: Hyaluronan synthase, HA: Hyaluronic acid, Tn-R: Tenascin-R 
nervous system of many higher organisms (Celio et al., 1998). Figure 1.1 shows a schematic of the PNN on the cell body of a neuron. Hyaluronic acid (HA), link proteins of the hyaluronic acid and proteoglycan link binding protein family (HAPLN), including HAPLN1, chondroitin sulfate proteoglycans (CSPG), and tenascin-R (Tn-R), assemble into a dense, lattice like sheet to form the PNN as seen in Figure 1.1. CSPG are highly anionic; they are comprised of lectican, also called hyalectan, protein cores covalently bound to varied numbers of chondroitin sulfate glycosaminoglycan (CS-GAG) chains via serine residues (Wang and Fawcett, 2012). There are five different lecticans in the PNN, aggrecan, neurocan, versican, phosphacan and brevican, which all have some conserved structure. The stereotypical lectican structure is an N-terminal G1 domain comprised of an immunoglobulin repeat and two link modules, a central GAG attachment region, a C-terminal G3 domain comprised of two epidermal growth factor (EGF)-like repeats, a C-type lectin domain, and a complementary regulatory protein (CRP)-like module are present in all of the lecticans (lozzo, 1998). The GAG chains consist of repeating disaccharide units of glucuronic acid (GlcA) and $N$ acetygalactosamine (GalNac). Despite the presence of these components of the PNN in utero, the PNN does not fully assemble into this configuration until the end of the third week of post-natal development in rodents, demonstrating that the PNN is developmentally regulated - older animals can have higher numbers of PNN+ neurons compared to younger animals. The maturation of the PNN on populations of these fastspiking neurons, neurons with action potentials that fire with very high frequency without really slowing down and they can recover rapidly (Izhikevich, 2003), is closely timed with the closure of critical periods of development, 
times of enhanced plasticity in post-natal development, and may regulate closing these epochs of high neural flexibility (Alberini and Travaglia, 2017; Hou et al., 2017).

Exit from the critical period of development coincides with the complete, assembled PNN (Hensch, 2005; Nabel and Morishita, 2013). The critical period is present in the senses like vision and audition and processes like learning and memory. Each of these functions have their own critical period. For example, a child may exit the critical period for vision around age 4 but remain within the critical period for hippocampal-dependent learning until around 8 years old. Indeed, in rodent models, the PNN may be present in the brain circuitry responsible for one modality (e.g., audition or vision) days before it is detectable within another brain region in the same animal. Interestingly, before animals have a fully-formed PNN, it appears that they do not learn or recall training like adult animals (Brown and Freeman, 2014; Schreurs et al., 2013). In addition, animals without a PNN have been observed to express behavioral phenomena such as impaired context learning and facilitated extinction of the aversive stimulus. Context learning involves animals forming associations between their training and the environment in which their training took place (for example, a rodent associating the chamber they received foot shock in with the foot shock itself) (Bouton, 2004; Lopez et al., 2012; Scott et al., 2015). Young animals do not learn fear conditioning like rodents that are beyond the critical period; older animals can acquire training and retain the aversive training even after undergoing extinction training (Alberini and Travaglia, 2017). Older animals lacking 
a PNN are more likely to undergo extinction at a more rapid rate, i.e. facilitated extinction, than those with an intact PNN. These differences in behavioral responses correlate with PNN structures as rodents within the critical period have a juvenile, diffuse PNN and older animals that have exited the critical period have a mature, dense PNN. This relationship is shown in Figure 1.2.

Due to its relationship with the critical period, the PNN has been implicated as a possible storage mechanism for memories, including long-term memories (Tsien, 2013), the aversive memories related to post-traumatic stress disorder (Gogolla et al., 2009; Thompson et al., 2017), and the hedonic memories associated with opioid use (Lasek et al., 2017; Slaker et al., 2016). Animals early in postnatal development have minimal to no PNN and have similar behavior to those that

PNN, Plasticity, and Age during Normal Development

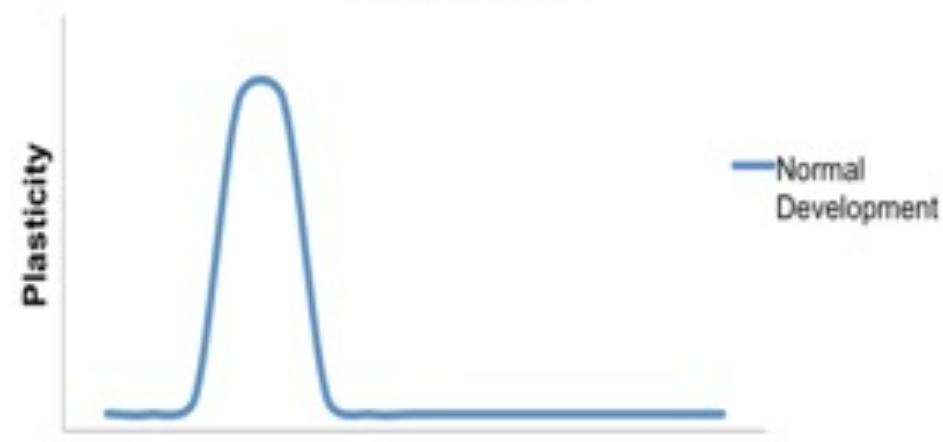

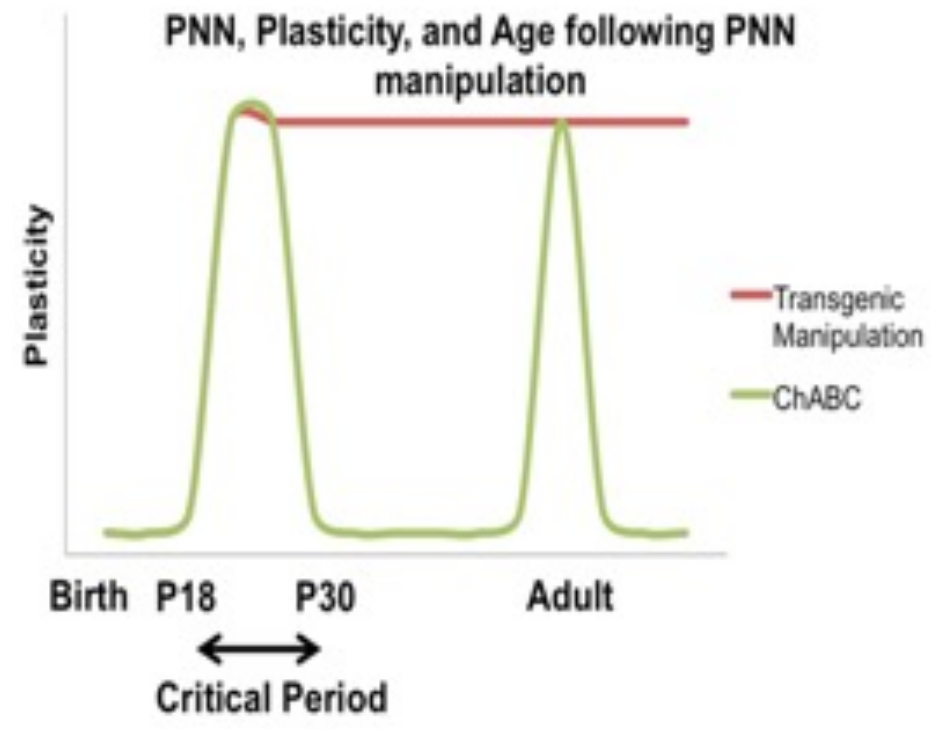

Figure 1.2. PNN, plasticity, development, and manipulation. The top panel shows plasticity as a rodent develops normally. The animal exits the critical period around the same time the PNN matures in rodents. The bottom panel shows that animals with transgenic manipulations do not exit the critical period on the red line. When ChABC is used to degrade the PNN, it elevates plasticity back to levels seen in the critical period in the adult animal. Figure modified from https://doi.org/10.1093/ib/mvu067 
have had enzymatic manipulations of the PNN, suggesting disruption of or the absence of the PNN profoundly affects learning and memory. Enzymatic degradation of the PNN or manipulation of PNN components in transgenic models can functionally revert adult animals to a heightened level of plasticity seen during the critical period, i.e. to a state associated with younger animals sometimes referred to as infantile or iPlasticity (Gogolla et al., 2009; Miyata and Kitagawa, 2016; Xue et al., 2014). Enzymatic degradation relies upon a bacterial enzyme, chondroitinase $A B C$ (ChABC), which is capable of temporarily disrupting the PNN; a bacterial enzyme that catalyzes chondroitin sulfate degradation by removing the 1,4- $\beta$-hexosaminyl linkages in CSPG (Brückner et al., 1998; Deepa et al., 2006; Koppe et al., 1997; Massey et al., 2006).

\subsection{PNN and Learning and Memory}

\subsubsection{The PNN is altered in diseases affecting Learning and Memory}

The PNN has been implicated in storing affective, or strongly emotional memories; evidence suggests that dysfunction of the PNN could be involved in diseases related to a breakdown of learning and memory (Carbo-Gas et al., 2017; Gogolla et al., 2009; Lasek et al., 2017; Slaker et al., 2015, 2018; Thompson et al., 2017; Tsien, 2013; Xue et al., 2014). For example, the PNN has been associated with storing long-term memories and defective PNN function could play a role in diseases like Alzheimer's disease (AD). AD is the most common form of dementia and is thought to arise from an increase in the amyloidogenic pathway processing amyloid precursor protein and from pathologies of the microtubule associated protein tau (de Calignon et al., 2012; Selkoe and Hardy, 2016). There is a correlation between the tau pathology associated with $A D$ and abnormal 
formation of HA, the backbone of the PNN, in a murine model (Li et al., 2017). In this transgenic mouse model of tauopathy, expressing a human mutant gene resulted in significant decreases in the expression of hyaluronic acid synthase 1 (HAS1). In this model, several properties of HA production, including length of HA chains and rate of HA production, were altered as a result of decreased HAS1 activity. In the hippocampus, one of the first and most severely affected sites of neurodegeneration in $A D$ (de Calignon et al., 2012; Nakamura et al., 2012; Passamonti et al., 2017; Pennanen et al., 2004; Shankar et al., 2008), three isoenzymes, HAS1, HAS2, and HAS3, synthesize HA of different sizes and at varying rates. In addition, postmortem human samples from normal, mild $A D$, and severe $A D$ brains exhibit a different prevalence of each isoenzyme. The mild AD brain had the greatest number of HAS1-positive plaques and the severe AD brain also had significantly increased numbers of HAS1-positive plaques compared to normal brain samples (Li et al., 2017). Although the mouse model showed a decrease in HAS1 while the human tissue had an increase, changes in the relative abundance of these isozymes could lead to an aberrant PNN that is built upon an atypical HA backbone. Other studies have found that proteoglycans, including CSPG, are found in amyloid beta plaques (DeWitt et al., 1993), may be involved in its uptake into neurons (Wesén et al., 2018), and may have altered structure as a result of AD (Zhang et al., 2016). These results indicate that an abnormal PNN could be a marker for AD (Végh et al., 2014) and that the PNN could be an alternative therapeutic focus for AD drugs (Suttkus et al., 2016; Yang et al., 2017). Another study found that a transgenic mouse model of AD led to reduction of parvalbumin-positive $(\mathrm{PV}+)$ interneurons and $\mathrm{PV}+$ neurons surrounded by a PNN in hippocampal subfields (Cattaud et al., 2018). These changes were observed 
even in animals as young as 3 months of age, suggesting PNN breakdown in the hippocampus precedes memory loss. Exposing mice to an enriched environment for 10 weeks led to a rescue of the $\mathrm{PV}+/ \mathrm{PNN}+$ neurons and may be related to the other studies that found early exposure to an enriched environment improved memory performance in the same mouse model of AD (Verret et al., 2013). Enriched environment is a method used to manipulate a rodent's environment, usually by providing toys or other accessories, in order to provide social, physical, and sensory stimulation. Environmental enrichment is known to promote neurogenesis in brain regions associated with learning and memory.

In addition to $A D$, the PNN may also have a role in cognitive difficulties related to depression. A social-defeat model was used to induce a chronic depressive-like state in rats, which resulted in increased production of several components of the PNN in the hippocampus. Social-defeat is a paradigm using social conflict between members of the same species to induce stress and is used to model depression. A dominant male is placed into a resident animal's cage and will attack the resident rodent, forcing it to submit. After a period of time, the intruder may be physically separated from the resident but remain visible to the resident to cause further stress; this experiment may also be called a resident-intruder test. The changes induced by social-defeat were associated with decreased inhibitory communication in the hippocampal population of PV+ interneurons as well as significantly poorer performance on object place recognition, a memory task dependent upon the hippocampus. PV+ interneurons are often PNN+ (Baker et al., 2017; Favuzzi et al., 2017; McDonald et al., 2017; Morris and Henderson, 2000; Mueller et al., 2016; Wen et al., 2017). Administering imipramine, an anti-depressant, systemically 
rescued the abnormal increases in the PNN (Riga et al., 2017). Stereotaxic infusion of ChABC was used as a direct approach to acutely alter the PNN. ChABC infusion in this depressive model rescued hippocampal inhibitory transmission and improved memory performance (Riga et al., 2017). By targeting the PNN, it could be possible to alleviate some of the cognitive issues faced by those with depression.

Learning and memory processes associated with pleasurable experiences and reward are hijacked in the presence of addictive substances like opioids and other narcotics. These substances induce extremely hedonic feelings and the memories associated with those feelings of elation are so intense they may drastically alter the user's behavior. The PNN in the cerebellar cortex, particularly around the Golgi cells, a type of inhibitory cell that release inhibitory neurotransmitters like gamma-aminobutyric acid (GABA) and glycine onto granule cells and unipolar brush cells (Bazzigaluppi et al., 2013; Jörntell, 2016; Sillitoe et al., 2012), may maintain drug-related memories connected to cocaine use. A Pavlovian conditioning paradigm, cocaine-induced place preference conditioning, is a behavioral test where one environment is associated with drug treatment and a different environment with the absence of the drug. Rodents are allowed to choose which environment they prefer, which provides insight into physiological responses in the brain in response to drug exposure and conditioning, and how therapeutics may prevent or reduce drug-seeking behaviors (Huston et al., 2013; Koob, 2008; Koob and Le Moal, 2001). Changes to the PNN were observed in rats exposed to cocaine-induced place preference conditioning (Blacktop et al., 2017; Carbo-Gas et al., 2017). Interestingly, the effects upon the PNN differed in different regions of the brain. Increased expression of PNN proteins was observed in the cerebellar cortex but reduced 
PNN protein expression was observed in one of the deep cerebellar nuclei (DCN), the medial cerebellar nucleus. Neurons expressing c-fos, which can be a marker of neural activity (Hoffman et al., 1993) and cocaine use (Graybiel et al., 1990), were also increased in the cerebellar cortex, following cocaine-induced preference, particularly in lobule 8 and 9 (Carbo-Gas et al., 2017). The increase in c-fos expression further supports the idea that alterations to the PNN are correlated to activity. The PNN may be a target to prevent drug relapse following cessation as PNN breakdown lowers drug seeking behaviors in animal models of relapse (Blacktop et al., 2017; Xue et al., 2014). Targeting the PNN for controlled degradation may prevent relapse of those struggling with addictive disorders.

These results show that alterations to the PNN occur in disease and conditions resulting in memory disorders. Although many of these results are correlative, they suggest that the PNN is an important structure that can influence learning, memory, and behavior in diseases related to learning and memory. The experimental evidence outlined below demonstrates that alterations in the PNN can alter learning and memory, providing proof-of-principle that the PNN is potentially a therapeutic target for the treatment of these and other diseases.

Although the current work may seem constrained to the bench, there may be possible clinical correlations for PNN manipulations. Doxycycline, a commonly prescribed member of the tetracycline class of antibiotics, is capable of crossing the blood-brain barrier. In addition to be being an antibiotic, doxycycline is also capable of inhibiting the activity of some matrix metalloproteinases (MMP). MMPs are capable of manipulating the PNN by enzymatically cleaving the components within the PNN. By inhibiting MMP 
remodeling of the PNN, it is possible to prevent the alterations associated with memory formation. Indeed, doxycycline has been found to diminish recall of aversive fear conditioning in humans. Human subjects who were given doxycycline during acquisition had decreased threat memory compared to placebo controls. Doxycycline subjects also experienced greater surprise when re-exposed to the conditioned stimulus, demonstrating they had forgotten the stimulus and were re-learning it (Yang et al., 2017). These results suggest that doxycycline and other MMP inhibitors can be used to manipulate the PNN and prevent retention of fear memories (Bach et al., 2019, 2017; Burhans et al., 2013). Other work has found that certain antidepressants (Deschaux et al., 2013; Guirado et al., 2014; Umemori et al., 2018, 2015) or anticonvulsants (Gervain et al., 2013) can diminish mature PNN to reopen the critical period. Since these drugs are already approved for human use, they may prove useful as therapeutics for other disorders affecting learning and memory such as post-traumatic stress disorder, addiction, $A D$ and depression.

\subsubsection{PNN Manipulations and Learning and Memory}

The PNN's function in memory storage was established using experimental methods such as enzymatic degradation, to alter the components forming the PNN. Infusion of ChABC allows comparison of responses in the presence (control) and disruption (ChABC treated) of an intact PNN. This strategy was applied to investigate the role of the PNN in storing memories generated during fear conditioning $(\mathrm{FC})$ to exposure to uncued foot-shock: Figure 1.2 shows how ChABC reinstates the plasticity observed in the critical period in adulthood. FC is an associative experimental paradigm where an innocuous stimulus (a tone or light), the conditioned stimulus (CS), is paired with an 
aversive stimulus (foot shock, eyelid shock), the unconditioned stimulus (US). Subjects innately have a response, the unconditioned response (UR) to the US. After repeated pairings of the tone or light and shock are presented, the subject will begin expressing an anticipatory or conditioned response (CR) to the CS. This is evidence that the subject has learned that the CS and US are paired together (Molchan et al., 1994; Woodruff-Pak, 1997). Mice were given bilateral stereotaxic infusions of ChABC to the basolateral amygdala, an important brain region for processing fear (LeDoux, 2003, 2014, 2000; LeDoux et al., 1988; Medina et al., 2002; Phillips and LeDoux, 1992), prior to the footshock conditioning. Animals receiving $\mathrm{ChABC}$ acquired the aversive training and exhibited no differences from controls during the training session. However, when the ChABC-infused and control animals underwent extinction training, a protocol designed to train the animal that the previously aversive association is no longer valid, the ChABCinfused mice had facilitated extinction (stopped responding to the shock at a faster rate) compared to the other animals (Gogolla et al., 2009). Extinction is a behavioral protocol and phenomenon (Bouton, 2004; Ganella et al., 2018; Herry et al., 2010; Maren and Holmes, 2016; Monfils et al., 2009; VanElzakker et al., 2014). Extinction as a protocol can involve presenting the CS alone to an animal conditioned with CS-US pairings. After repeated CS-only presentations, the level of CRs will return to near baseline (Rogers et al., 2001; Steinmetz et al., 1989). The phenomenon is context-dependent and involves the animal learning new information (CS does not precede US) that inhibits the old information (CS precedes US). In addition, these animals in the Gogolla study did not display common extinction phenomenon like spontaneous recovery, where the extinguished conditioning returns after time has passed (Rescorla, 2004), but were still 
susceptible to context-dependent emergence of the response when tested in the context used during FC (Gogolla et al., 2009). This seminal work in the PNN literature by Gogolla showed that ChABC enzymatic activity alters the PNN of adult mice to appear more like the PNN of young mice within their critical period and that this restoration of the critical period of plasticity has profound effects on fear behaviors. It is noteworthy that the infusion of ChABC only had significant effects on extinction if it was infused prior to foot-shock conditioning; mice receiving infusions following training did not show extinction of the fear response, indicating that degrading the PNN prevents fear memories from being stored or is crucial for the mechanisms underlying extinction learning (Gogolla et al., 2009). Another study found that components of the PNN change during conditioning. Lectican mRNA levels increase and peak $4 \mathrm{hr}$ following auditory FC and elevated expression continues for at least 24 hours post-training. A commonly used stain to detect the PNN, Wisteria floribunda agglutinin (WFA) was used to visualize the PNN. WFA is a plant lectin that binds to the $\mathrm{N}$-acetylgalactosamine residues in CSPG and is one of the most widely used markers for the PNN. WFA reactivity also increases in the auditory cortex at $4 \mathrm{hr}$ post-FC, which again persisted for a day before returning to baseline. Control animals did not show these changes in their auditory cortex. ChABC was administered to these animals $72 \mathrm{hr}$ before $\mathrm{FC}$ and their response to $\mathrm{FC}$ and extinction training assessed as the amount of freezing. Freezing is a behavioral phenomenon observed in many species, including rodents and humans. Animals experiencing a threat will become immobile and "freeze" in the presence of the threat. Rodents will also freeze to the CS or context after FC. If animals stop freezing as often or all together when exposed to the CS, it suggests they no longer fear the CS because they do not associate it with the aversive US and may 
have learned new information that supersedes the old, the CS does not precede the US. ChABC infusion had no effect on acquisition or early fear extinction, i.e. the first few trials of an extinction period, however, at later times ( 24 and 48 hours after FC), ChABCtreated mice froze significantly less compared to vehicle control-treated mice, but these effects seem to be time-dependent. When ChABC was administered shortly after FC, there was no effect on fear extinction when tested acutely. However, significant decreases in extinction were seen 1- 2 days following enzymatic manipulation (Banerjee et al., 2017).

While FC investigates the formation, storage, and removal of noxious stimuli, there are potent and dangerous memories associated with feeling good, like addictive substances. Exposing rats to various opioids and infusing ChABC to amygdaloid nuclei, alleviates drug-priming behavior (Xue et al., 2014). ChABC was directly infused into both the basolateral amygdala and the central nucleus of the amygdala after completing conditioned place preference training to morphine and cocaine. Rodents receiving ChABC to these regions also were less likely to self-administer heroine during a drug priming or relapse test. Drug priming behavior can be observed in people when an abstinent but previous drug user relapses and now expresses greater desire to use again after this reintroduction. To model this, animals are re-exposed to the drug following cessation to mimic the initial relapse. The animals subsequently exhibit elevated motivation to obtain the next dose (de Wit, 1996). Since drug priming is an animal model of drug relapse, this result lends credibility to targeting the PNN to aid recovery from drug addiction. Interestingly, the reduced priming effects were only seen in the presence of addictive drugs and not naturally hedonic stimuli like food rewards. Only the ChABC- 
infused rats that also underwent extinction training had significant changes to AMPAR subunits; the alpha-amino-3-hydroxy-5-methyl-4-isoxazolepropionic acid (AMPA)receptor is an ionotropic receptor for glutamate capable of altering neuron excitability by controlling the influx of calcium and sodium ions. These results were not observed in animals that were only given ChABC or control animals that underwent extinction (Xue et al., 2014).

ChABC infusions also altered dendritic spines, which can change their shape and turnover as a result of learning, recalling information, and cognition (Frankfurt and Luine, 2015; McCann and Ross, 2017). Interestingly, degradation of the PNN alone was enough to cause changes to the dendritic spines in the amygdala. Rodents receiving ChABC infusions to the amygdala exhibit significant reductions in large dendritic spine numbers and in some cases total spine number. Additionally, a day after extinction training, rodents receiving ChABC had fewer dendritic spines than control animals. The rodents undergoing extinction and enzymatic manipulation had fewer total numbers of spines per unit length in general and fewer large spines as well (Pignataro et al., 2017). These results indicate that the PNN may stabilize dendritic spines on proximal dendrites and removal of the PNN via enzymatic degradation promotes spine remodeling or elimination. It is difficult to determine if ChABC infusion alone can cause these effects or if the combination of $\mathrm{ChABC}$ infusion with extinction training led to the drastic reduction of dendritic spines. Perhaps the PNN maintains spine number and morphology in the mature brain and the dynamic nature of dendritic spines in early life reflects the absence of the dense PNN seen in adults. In neurotypical post-mortem human brain tissue the number of dendritic spines peaks in early childhood (between 2-7 yr old) but modification 
to spines continues well into early and middle adulthood (Petanjek et al., 2011). This coincides with the formation of the PNN in the hippocampus of humans at around $8 \mathrm{yrs}$ of age with little change into adulthood (Rogers et al., 2018). In the basolateral amygdala of rats, the number of dendritic spines grows as the animals exit a critical period, between P20-P35. There are no significant changes in spine number between timepoints outside of the critical period (Koss et al., 2014).

\subsection{Genetic manipulation of the PNN}

Genetic approaches also support a critical role for CSPG and the PNN in controlling neuronal morphology and in learning and memory. Transgenic manipulations of the PNN result in persistent plasticity and animals may remain in a critical period throughout their lifespan (Figure 1.2). The sulfation pattern of CSPG changes temporally and is controlled by the activity of two chondroitin sulfotransferases, chondroitin 6sulfotransferase 1 (C6ST1) and chondroitin 4-sulfotransferase 1 (C4ST1). C6ST1 is much more active early in postnatal development while C4ST1 is

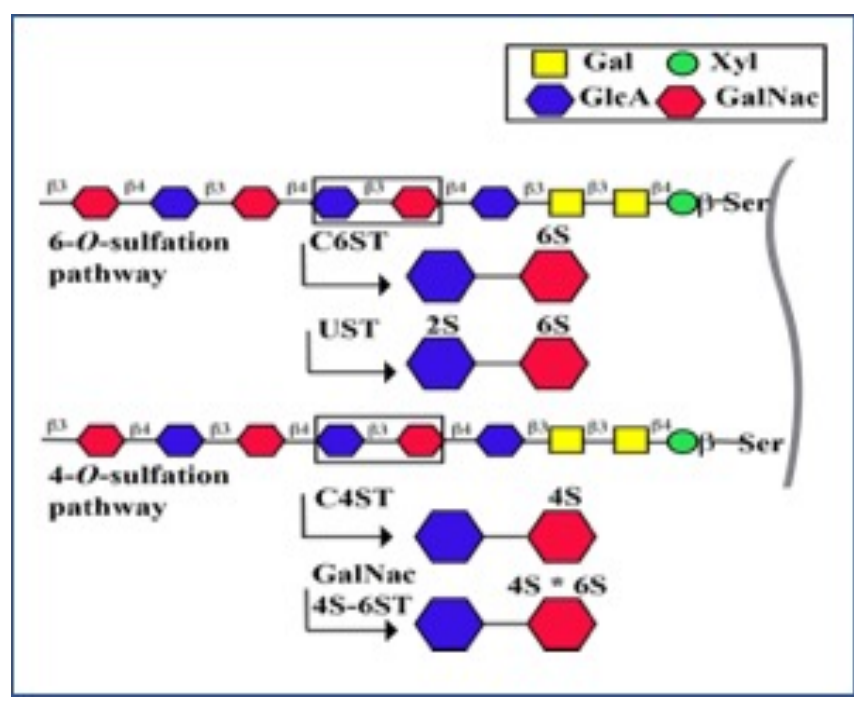

Figure 1.3. CSPG structure and formation.

The figure shows the structure of the chondroitin sulfateglycosaminoglycan (CS-GAG) chain component of CSPG. An attachment region connects the CS-GAG chain to a lectican core (shown as grey line). Alternating GlcA and GalNac residues are added to the chain. The GalNac residue can be sulfated at the $6^{\text {th }}$ or $4^{\text {th }}$ oxygen by two enzymatic pathways that are active at different developmental stages. The 6-O-sulfation pathway is active in very young animals while the 4-O-sulfation pathway becomes more prevalent as the animal ages into and throughout adulthood.

Gal: Galactose, GlcA: Glucuronic acid, Xyl: Xylose, GalNac: N-acetylgalactosamine, Ser: Serine, C6ST1: Chondroitin 6-sulfotransferase 1, C4ST1: Chondroitin 4sulfotransferase 1 
predominantly active in adulthood. The two different CSPG synthesis pathways generate a structurally different PNN and are shown in Figure 1.3. Transgenic expression of C6ST1 beyond its normal window of expression, which is early in postnatal development, altered the structure of the PNN and altered biological function extending the critical period. Adult transgenic mice overexpressing C6ST1 lack dense PNN around neurons in the primary visual cortex. The PNN in adult C6ST1 transgenic mice appeared more diffuse and more closely resembled the PNN of preweanling mice still within the critical period and transgenic adults remain susceptible to monocular deprivation, a method of limiting experience-dependent plasticity in the visual cortex of young animals (Miyata et al., 2012; Miyata and Kitagawa, 2016). These experiments indicate that modifying the PNN by either ChABC enzymatic digestion or C6ST1 overexpression can induce another epoch of plasticity where activity robustly modulates cortical circuitry. CSPG sulfation status may also be related to diseases like schizophrenia. The hippocampi from postmortem schizophrenic brains were compared to age-matched control. Western blots found a significantly higher intensity of CSPG in the 4-S position in the schizophrenic hippocampi that could not be accounted for by post-mortem interval, storage time, or age (Yukawa et al., 2018). Manipulation of expression of other PNN components, the link proteins that connect CSPG to HA, also alters PNN structure and function. Knockout of Bral2/HAPLN4, which is found at high levels in the brainstem and cerebellum, robustly reduced the levels of brevican, a lectican in CSPG, at these sites. Neurocan, another lectican, and Crtl1, another link protein, were undetectable in the deep cerebellar nuclei and some auditory structures of the KO mice; WFA and HA reactivity were also diminished in these select brain areas. Electron microscopy imaging of brains from Bral2 
KO mice demonstrated that these animals had fewer synapses in the deep cerebellar nuclei compared to control animals (Bekku et al., 2012). These results suggest that Bral2 is required for brevican to become incorporated into the PNN and loss of this link protein prevents normal PNN formation in the cerebellum and brainstem regions. However, it is unknown if brevican is still expressed but below the level of detection. The abnormal PNN seen in Bral2 KO mice could in turn reduce synapses in the affected areas.

\subsection{Functional connections and activity are required for normal PNN development}

Functional connectivity between neurons is necessary for normal PNN formation (Lin et al., 2018), suggesting that the PNN of some neuron populations are involved in synaptic development and neurotransmitter release. Connections between Purkinje cells and neurons in the DCN are required for formation and maintenance of a normal PNN. In a mouse model of Purkinje cell degeneration, cells in the DCN lacking the connection to the Purkinje cell axon had an imbalance of the excitatory and inhibitory synaptic terminals compared to wild-type mice (Blosa et al., 2015). Animals with degenerated Purkinje axons had fewer GABAergic synapses on DCN neurons and these alterations correlated with reduced brevican and HAPLN4/ Bral2 intensity. Speculatively, without GABAergic input, a cell may construct an irregular PNN lacking one or more components. As described above (Bekku et al., 2012), reduced Bral2 expression may alter the incorporation of brevican into the PNN since link proteins attach lecticans to the HA backbone. In addition, deletion of HAPLN4/Bral2 has been found to be responsible for maintaining the inhibitory synapse made between the Purkinje cells and the large excitatory DCN neurons (Edamatsu et al., 2018). 
Gelatinases including matrix metalloproteinase-9 (MMP-9) can play a role in the normal synaptic and behavioral plasticity in neural circuit remodeling (Bach et al., 2017) . MMPs are known to proteolytically cleave collagen (Van den Steen et al., 2002), but also degrade aggrecan and the link proteins (Fosang et al., 1992; Nguyen et al., 1993), and thus could impact the structure of the PNN. Stimuli that induce late-long term potentiation (L-LTP), a form of synaptic plasticity, also activate MMP-9; this can be observed following chemical induction and strong tetanic stimulation capable of inducing LTP (Bach et al., 2017). The active MMP-9 is localized to synapses and its activation can be blocked with NMDAR antagonists, indicating that activity is needed to activate MMP-9. In addition, the use of broad-spectrum MMP inhibitors can also block the induction of both LTP and longterm depression. MMP-9 can even change synaptic structures without LTP-inducing stimuli. Local application of proteolytically active MMP-9 to dendrites can lead to structural changes. This includes morphological changes indicative of increased synaptic strength at synapses, for example more receptors inserted at the spine, and enlarged dendritic spines that peak shortly following application. However, prolonged exposure to MMP-9 generates the opposite effects. Cultures of hippocampal neurons exposed to MMP-9 for 90 minutes have elongated, slender dendritic spines with diminished spine heads (Huntley, 2012). The effects of MMP-9 on molecular and morphological changes associated with memory may be related to its ability to manipulate the PNN at the synapse since this enzyme can degrade the extracellular matrix and PNN.

Patients with neurodevelopmental diseases like Fragile X Syndrome and Autism Spectrum Disorder typically have both learning delays and sensory deficits in several modalities. A transgenic mouse model of Fragile $\mathrm{X}$ Syndrome, KO of Fmr1, exhibited 
elevated MMP-9 activity in the auditory cortex during the critical period of auditory development. This was associated with abnormal GABAergic inhibitory cell development and a diminished PNN was observed around these cells. In vitro slice recordings showed that these inhibitory cells had altered spiking behavior and were more likely to have spontaneous firing and firing in response to sound presentation. Returning MMP-9 levels back to normal levels rescued all of these effects (Wen et al., 2017). Although the authors did not investigate the role of MMP-9 in learning and memory, it potentially plays a role in the cognitive delays associated with Fragile X. In addition to autism like disorders, MMP9 as well an MMP regulator, tissue inhibitor of metalloproteinases-1 (TIMP-1) may play a role in several dementia-related diseases. When compared to healthy, age-matched controls, MMP-9 protein levels were significantly lower in those who had frontotemporal dementia (Tuna et al., 2018). Frontotemporal dementia is a heterogeneous neurodegenerative disease that affects the frontal and temporal lobes. Symptoms are variable and can cause issues in motor skills, language, executive functioning, and behavior (Olney et al., 2017). Protein levels of TIMP-1 were also lower in the brains from patients with frontotemporal dementia as well as AD brains compared to the control group. These findings suggest that MMP-9 and TIMP-1 play a role in the degeneration associated with these two dementias.

MMP-9 may also be influenced by external stimuli. The effect of an enriched environment on MMP-9 was examined in mice and significantly increased MMP-9 levels were seen in the cerebellar cortex, but there were no observable differences to the PNN in wild-type animals compared to animals reared in standard environment. MMP-9 knock out mice reared in the enriched environment had elevated PNN staining in the deep 
cerebellar nuclei compared to wild-type mice. Elevations in excitatory synaptic markers were also seen in the MMP-9 knock out (Stamenkovic et al., 2017). Thus, elevated MMP9 in response to an enriched environment appears responsible for constraining changes in the PNN and neuronal connections. However, the effect of changes to MMP-9 upon the PNN in the cerebellum and upon learning and memory of cerebellar-dependent tasks remain unexplored. Since there were changes to the synaptic receptors, these relationships are an important line of future investigation.

\subsection{General Mechanisms of PNN Control}

The evidence from the ChABC and transgenic manipulations to the PNN demonstrates that density (dense mature or diffuse immature) and composition of the PNN regulates neural plasticity. The PNN has been postulated to control neural plasticity via multiple mechanisms (Sorg et al., 2016). The simplest mechanism is by acting as a physical barrier and preventing extending neurites and axons from making contact onto a PNN-positive cell. Thus, an intact, mature PNN would prevent healthy and injured neurons in the CNS from making new connections. Enzymatic breakdown of the PNN would allow these neurons to make new contacts, even repairing connections following damage to the neural tissue (Miyata and Kitagawa, 2016; Xue et al., 2014). The PNN can also act as a scaffold for chemo-repulsive molecules. Semaphorin 3A, a molecule that repels axons, can associate with the PNN of inhibitory interneurons in both the neocortex and the cerebellum (Carulli et al., 2013; de Winter et al., 2016). Dense PNNs acting as inhibitory scaffolds could prevent extending neurites from contacting $\mathrm{PNN}+$ cells by chemorepulsion. By degrading the PNN and removing the binding sites for 
Semaphorin 3A, it may be possible for new connections and additional contacts to form. These mechanisms are not mutually exclusive and could be context dependent. It would be interesting to distinguish the barrier effects of the PNN from the effects of the chemorepulsive molecules that may be embedded within. The PNN can also corral synaptic receptors, like AMPA receptors, at sites of high synaptic activity, perhaps preventing them from undergoing endocytosis. Decreased rates of AMPAR endocytosis are associated with learning and memory, indicating that stabilized pools of AMPAR at synapses are a key step of learning and memory (Anggono and Huganir, 2012; Derkach et al., 2007). Others have also established that the PNN may be important in maintaining the membrane capacitance of ensheathed neurons (Tewari et al., 2018). The cell membranes of neurons and other excitable cells are capable of acting as parallel-plate capacitors and storing charge. This permits neurons to fire as membrane voltage and resistance changes in time and space. This seems to be especially true for PNN+ interneurons compared to excitatory pyramidal cells. Thus, disrupting the PNN could alter the spiking activity of PNN+ neurons rendering them more likely to fire.

\subsection{Concluding Remarks}

Questions still remain about the PNN. Some areas of the CNS with PNN+ neurons have been well researched while other areas are quite understudied. In addition, the majority of PNN work has been done with rodents; study of the PNN in other animal species would provide more information about the maturation and distribution of the PNN. Additionally, although human tissue is difficult to obtain, studying how the PNN is changed in the human brain throughout development in both healthy brains and diseases affecting 
learning and memory should also be undertaken. These results would validate studies done with animal models or minimize the relevance of studies without translational merit. Performing causal studies in vitro or in vivo could provide support to the correlative work that comprises the majority of PNN studies. The majority of PNN studies focus on early postnatal development but investigations of aged animals are also worthwhile. Studies of older animals could be used to understand how the PNN is related to normal aging as well as diseases affecting elderly populations. An inducible transgenic manipulation would also be useful. Current transgenic manipulations of the PNN are primarily focused on how these manipulations alter the PNN during post-natal development. Using drugs, like tamoxifen, or virally inducible transgenic in adult animals would allow the PNN to develop normally and then precisely remove one of the PNN compounds in adulthood. Multiple mechanisms have been proposed for the PNN, but no definitive consensus has emerged at this time. In order to determine the mechanism combining molecular techniques and behavior could provide greater insight into how the PNN modulates learning and memory.

Although it appears clear that the PNN exerts control over neural plasticity, the definitive molecular mechanism remains elusive. The evidence suggests that there may be multiple mechanisms through which the PNN influences neural plasticity throughout the development of an organism. The processes of learning and memory rely heavily upon changes at the synapse and plasticity throughout development. In addition to changes to the synapses, the PNN can alter neuronal membrane excitability as well (Bekku et al., 2012; Chu et al., 2018; Edamatsu et al., 2018; Hirono et al., 2018; Lensjø et al., 2016; Slaker et al., 2018; Tewari et al., 2018), another crucial factor underlying 
learning and memory (Booth et al., 2016; Narayanan and Johnston, 2007; Thompson et al., 1996; Wang et al., 2018; Wang and Schreurs, 2014, 2010). During the critical period, it may be possible to reorganize preexisting cortical circuitry by manipulating the PNN. Manipulation of the CSPG or other components can restructure the PNN, thus altering the time period in which the critical period is open, or "reopening" the critical period and restoring neuronal plasticity. While studies using transgenic models have provided crucial insights into the significance of specific components on the structure and function of the PNN, these models have yet to be exploited to address the effects upon learning or memory. The limitation of the transgenic models thus far, is the perturbation of the PNN throughout life. ChABC remains the standard approach for perturbing the PNN after it has been fully established, but the limitation of the approach is the indiscriminate disassembly of the PNN. Additional conditional transgenic models targeting individual link proteins, HAS isoenzymes, enzymes involved in CSPG formation, and the various lecticans at different stages of development will provide crucial insights into the role of each in the assembly of the PNN. Perturbation of specific PNN components in mature animals will define which components are important for the maintenance of the PNN and regulation of neural plasticity and may be additional targets for the development of therapeutics. Further development of models targeting specific cell populations, e.g. PV+ neurons or Purkinje cells, will provide refined insight into the role of individual components of the PNN on these specific cells and their influence upon learning and memory. As the body of work on PNN grows, it will be soon possible to understand how this specialized ECM regulates processes related to learning and memory. 
References:

Alberini, C.M., Travaglia, A., 2017. Infantile Amnesia: A Critical Period of Learning to Learn and Remember. J. Neurosci. 37, 5783-5795.

https://doi.org/10.1523/JNEUROSCI.0324-17.2017

Anggono, V., Huganir, R.L., 2012. Regulation of AMPA receptor trafficking and synaptic plasticity. Curr. Opin. Neurobiol. 22, 461-469. https://doi.org/10.1016/j.conb.2011.12.006

Bach, D.R., Näf, M., Deutschmann, M., Tyagarajan, S.K., Quednow, B.B., 2019. Threat Memory Reminder Under Matrix Metalloproteinase 9 Inhibitor Doxycycline Globally Reduces Subsequent Memory Plasticity. J. Neurosci. 39, 9424-9434. https://doi.org/10.1523/JNEUROSCI.1285-19.2019

Bach, D.R., Tzovara, A., Vunder, J., 2017. Blocking human fear memory with the matrix metalloproteinase inhibitor doxycycline. Mol. Psychiatry. https://doi.org/10.1038/mp.2017.65

Baker, K.D., Gray, A.R., Richardson, R., 2017. The development of perineuronal nets around parvalbumin gabaergic neurons in the medial prefrontal cortex and basolateral amygdala of rats. Behav. Neurosci. 131, 289-303. https://doi.org/10.1037/bne0000203

Banerjee, S.B., Gutzeit, V.A., Baman, J., Aoued, H.S., Doshi, N.K., Liu, R.C., Ressler, K.J., 2017. Perineuronal Nets in the Adult Sensory Cortex Are Necessary for Fear Learning. Neuron 95, 169-179.e3. https://doi.org/10.1016/j.neuron.2017.06.007

Bazzigaluppi, P., Isenia, S.C., De Zeeuw, C.I., de Jeu, M.T.G., 2013. Chapter 8 - Gap Junctions and Neuronal Synchronization in the Olivocerebellar System, in: Dere, E. (Ed.), Gap Junctions in the Brain. Academic Press, San Diego, pp. 127-145. https://doi.org/10.1016/B978-0-12-415901-3.00008-6

Bekku, Y., Saito, M., Moser, M., Fuchigami, M., Maehara, A., Nakayama, M., Kusachi, S., Ninomiya, Y., Oohashi, T., 2012. Bral2 is indispensable for the proper localization of brevican and the structural integrity of the perineuronal net in the brainstem and cerebellum. J. Comp. Neurol. 520, 1721-1736. https://doi.org/10.1002/cne.23009

Blacktop, J.M., Todd, R.P., Sorg, B.A., 2017. Role of perineuronal nets in the anterior dorsal lateral hypothalamic area in the acquisition of cocaine-induced conditioned place preference and self-administration. Neuropharmacology 118, 124-136. https://doi.org/10.1016/j.neuropharm.2017.03.018

Blosa, M., Bursch, C., Weigel, S., Holzer, M., J\&\#xe4, Ger, C., Janke, C., Matthews, R.T., Arendt, T., Morawski, M., 2015. Reorganization of Synaptic Connections and Perineuronal Nets in the Deep Cerebellar Nuclei of Purkinje Cell Degeneration Mutant Mice. Neural Plast. 2016, e2828536. https://doi.org/10.1155/2016/2828536

Booth, C.A., Witton, J., Nowacki, J., Tsaneva-Atanasova, K., Jones, M.W., Randall, A.D., Brown, J.T., 2016. Altered Intrinsic Pyramidal Neuron Properties and Pathway-Specific Synaptic Dysfunction Underlie Aberrant Hippocampal Network Function in a Mouse Model of Tauopathy. J. Neurosci. Off. J. Soc. Neurosci. 36, 350-363. https://doi.org/10.1523/JNEUROSCI.2151-15.2016 
Bouton, M.E., 2004. Context and Behavioral Processes in Extinction. Learn. Mem. 11, 485-494. https://doi.org/10.1101//m.78804

Brown, K.L., Freeman, J.H., 2014. Extinction, reacquisition, and rapid forgetting of eyeblink conditioning in developing rats. Learn. Mem. Cold Spring Harb. N 21, 696-708. https://doi.org/10.1101//m.036103.114

Brückner, G., Bringmann, A., Härtig, W., Köppe, G., Delpech, B., Brauer, K., 1998. Acute and long-lasting changes in extracellular-matrix chondroitin-sulphate proteoglycans induced by injection of chondroitinase $A B C$ in the adult rat brain. Exp. Brain Res. 121, 300-310. https://doi.org/10.1007/s002210050463

Burhans, L.B., Smith-Bell, C.A., Schreurs, B.G., 2013. Subacute fluoxetine enhances conditioned responding and conditioning-specific reflex modification of the rabbit nictitating membrane response: implications for drug treatment with selective serotonin reuptake inhibitors. Behav. Pharmacol. 24, 55-64. https://doi.org/10.1097/FBP.0b013e32835d528e

Carbo-Gas, M., Moreno-Rius, J., Guarque-Chabrera, J., Vazquez-Sanroman, D., GilMiravet, I., Carulli, D., Hoebeek, F., De Zeeuw, C., Sanchis-Segura, C., Miquel, M., 2017. Cerebellar perineuronal nets in cocaine-induced pavlovian memory: Site matters. Neuropharmacology 125, 166-180. https://doi.org/10.1016/j.neuropharm.2017.07.009

Carulli, D., Foscarin, S., Faralli, A., Pajaj, E., Rossi, F., 2013. Modulation of semaphorin $3 A$ in perineuronal nets during structural plasticity in the adult cerebellum. Mol. Cell. Neurosci. 57, 10-22. https://doi.org/10.1016/j.mcn.2013.08.003

Cattaud, V., Bezzina, C., Rey, C.C., Lejards, C., Dahan, L., Verret, L., 2018. Early disruption of parvalbumin expression and perineuronal nets in the hippocampus of the Tg2576 mouse model of Alzheimer's disease can be rescued by enriched environment. Neurobiol. Aging 72, 147-158. https://doi.org/10.1016/j.neurobiolaging.2018.08.024

Celio, M.R., Spreafico, R., Biasi, S.D., Vitellaro-Zuccarello, L., 1998. Perineuronal nets: past and present. Trends Neurosci. 21, 510-515. https://doi.org/10.1016/S01662236(98)01298-3

Chu, P., Abraham, R., Budhu, K., Khan, U., De Marco Garcia, N., Brumberg, J.C., 2018. The Impact of Perineuronal Net Digestion using Chondroitinase ABC on the Intrinsic Physiology of Cortical Neurons. Neuroscience. https://doi.org/10.1016/j.neuroscience.2018.07.004

de Winter, F., Kwok, J.C.F., Fawcett, J.W., Vo, T.T., Carulli, D., Verhaagen, J., 2016. The Chemorepulsive Protein Semaphorin $3 \mathrm{~A}$ and Perineuronal Net-Mediated Plasticity. Neural Plast. 2016, e3679545. https://doi.org/10.1155/2016/3679545 de Wit, H., 1996. Priming effects with drugs and other reinforcers. Exp. Clin. Psychopharmacol., Relapse to Substance Abuse: Recent Findings From Basic and Clinical Research 4, 5-10. https://doi.org/10.1037/1064-1297.4.1.5

de Calignon, A., Polydoro, M., Suárez-Calvet, M., William, C., Adamowicz, D.H., Kopeikina, K.J., Pitstick, R., Sahara, N., Ashe, K.H., Carlson, G.A., Spires-Jones, T.L., Hyman, B.T., 2012. Propagation of Tau Pathology in a Model of Early Alzheimer's Disease. Neuron 73, 685-697. https://doi.org/10.1016/j.neuron.2011.11.033 
Deepa, S.S., Carulli, D., Galtrey, C., Rhodes, K., Fukuda, J., Mikami, T., Sugahara, K., Fawcett, J.W., 2006. Composition of Perineuronal Net Extracellular Matrix in Rat Brain A DIFFERENT DISACCHARIDE COMPOSITION FOR THE NETASSOCIATED PROTEOGLYCANS. J. Biol. Chem. 281, 17789-17800. https://doi.org/10.1074/jbc.M600544200

Derkach, V.A., Oh, M.C., Guire, E.S., Soderling, T.R., 2007. Regulatory mechanisms of AMPA receptors in synaptic plasticity. Nat. Rev. Neurosci. 8, 101-113. https://doi.org/10.1038/nrn2055

Deschaux, O., Zheng, X., Lavigne, J., Nachon, O., Cleren, C., Moreau, J.-L., Garcia, R., 2013. Post-extinction fluoxetine treatment prevents stress-induced reemergence of extinguished fear. Psychopharmacology (Berl.) 225, 209-216. https://doi.org/10.1007/s00213-012-2806-x

DeWitt, D.A., Silver, J., Canning, D.R., Perry, G., 1993. Chondroitin Sulfate Proteoglycans Are Associated with the Lesions of Alzheimer's Disease. Exp. Neurol. 121, 149-152. https://doi.org/10.1006/exnr.1993.1081

Edamatsu, M., Miyano, R., Fujikawa, A., Fujii, F., Hori, T., Sakaba, T., Oohashi, T., 2018. Hapln4/Bral2 is a selective regulator for formation and transmission of GABAergic synapses between Purkinje and deep cerebellar nuclei neurons. J. Neurochem. 147, 748-763. https://doi.org/10.1111/jnc.14571

Favuzzi, E., Marques-Smith, A., Deogracias, R., Winterflood, C.M., Sánchez-Aguilera, A., Mantoan, L., Maeso, P., Fernandes, C., Ewers, H., Rico, B., 2017. ActivityDependent Gating of Parvalbumin Interneuron Function by the Perineuronal Net Protein Brevican. Neuron 95, 639-655.e10. https://doi.org/10.1016/j.neuron.2017.06.028

Fosang, A.J., Neame, P.J., Last, K., Hardingham, T.E., Murphy, G., Hamilton, J.A., 1992. The interglobular domain of cartilage aggrecan is cleaved by PUMP, gelatinases, and cathepsin B. J. Biol. Chem. 267, 19470-19474.

Frankfurt, M., Luine, V., 2015. The evolving role of dendritic spines and memory: Interaction(s) with estradiol. Horm. Behav., Estradiol and cognition: molecules to mind 74, 28-36. https://doi.org/10.1016/j.yhbeh.2015.05.004

Ganella, D.E., Nguyen, L.D., Lee-Kardashyan, L., Kim, L.E., Paolini, A.G., Kim, J.H., 2018. Neurocircuitry of fear extinction in adult and juvenile rats. Behav. Brain Res. 351, 161-167. https://doi.org/10.1016/j.bbr.2018.06.001

Gervain, J., Vines, B.W., Chen, L.M., Seo, R.J., Hensch, T.K., Werker, J.F., Young, A.H., 2013. Valproate reopens critical-period learning of absolute pitch. Front. Syst. Neurosci. 7. https://doi.org/10.3389/fnsys.2013.00102

Gogolla, N., Caroni, P., Lüthi, A., Herry, C., 2009. Perineuronal nets protect fear memories from erasure. Science 325, 1258-1261. https://doi.org/10.1126/science.1174146

Graybiel, A.M., Moratalla, R., Robertson, H.A., 1990. Amphetamine and cocaine induce drug-specific activation of the c-fos gene in striosome-matrix compartments and limbic subdivisions of the striatum. Proc. Natl. Acad. Sci. U. S. A. 87, 6912-6916.

Guirado, R., Perez-Rando, M., Sanchez-Matarredona, D., Castrén, E., Nacher, J., 2014. Chronic fluoxetine treatment alters the structure, connectivity and plasticity of cortical interneurons. Int. J. Neuropsychopharmacol. Oxf. 17, 1635-46. http://dx.doi.org/10.1017/S1461145714000406 
Hensch, T.K., 2005. Critical period plasticity in local cortical circuits. Nat. Rev. Neurosci. 6, 877-888. https://doi.org/10.1038/nrn1787

Herry, C., Ferraguti, F., Singewald, N., Letzkus, J.J., Ehrlich, I., Lüthi, A., 2010. Neuronal circuits of fear extinction. Eur. J. Neurosci. 31, 599-612. https://doi.org/10.1111/j.1460-9568.2010.07101.x

Hirono, M., Watanabe, S., Karube, F., Fujiyama, F., Kawahara, S., Nagao, S., Yanagawa, Y., Misonou, H., 2018. Perineuronal nets in the deep cerebellar nuclei regulate GABAergic transmission and delay eyeblink conditioning. J. Neurosci. 3238-17. https://doi.org/10.1523/JNEUROSCI.3238-17.2018

Hoffman, G.E., Smith, M.S., Verbalis, J.G., 1993. c-Fos and related immediate early gene products as markers of activity in neuroendocrine systems. Front. Neuroendocrinol. 14, 173-213. https://doi.org/10.1006/frne.1993.1006

Hou, X., Yoshioka, N., Tsukano, H., Sakai, A., Miyata, S., Watanabe, Y., Yanagawa, Y., Sakimura, K., Takeuchi, K., Kitagawa, H., Hensch, T.K., Shibuki, K., Igarashi, M., Sugiyama, S., 2017. Chondroitin Sulfate Is Required for Onset and Offset of Critical Period Plasticity in Visual Cortex. Sci. Rep. 7, 12646. https://doi.org/10.1038/s41598-017-04007-x

Huntley, G.W., 2012. Synaptic circuit remodelling by matrix metalloproteinases in health and disease. Nat. Rev. Neurosci. 13, 743-757. https://doi.org/10.1038/nrn3320

Huston, J.P., Silva, M.A. de S., Topic, B., Müller, C.P., 2013. What's conditioned in conditioned place preference? Trends Pharmacol. Sci. 34, 162-166. https://doi.org/10.1016/j.tips.2013.01.004

lozzo, R.V., 1998. Matrix proteoglycans: from molecular design to cellular function. Annu. Rev. Biochem. 67, 609-652. https://doi.org/10.1146/annurev.biochem.67.1.609

Izhikevich, E.M., 2003. Simple model of spiking neurons. IEEE Trans. Neural Netw. 14, 1569-1572. https://doi.org/10.1109/TNN.2003.820440

Jörntell, H., 2016. Chapter 7 - Cerebellar Neuronal Codes-Perspectives from Intracellular Analysis In Vivo, in: Heck, D.H. (Ed.), The Neuronal Codes of the Cerebellum. Academic Press, San Diego, pp. 155-172. https://doi.org/10.1016/B978-0-12-801386-1.00007-1

Koob, G.F., 2008. A Role for Brain Stress Systems in Addiction. Neuron 59, 11-34. https://doi.org/10.1016/j.neuron.2008.06.012

Koob, G.F., Le Moal, M., 2001. Drug Addiction, Dysregulation of Reward, and Allostasis. Neuropsychopharmacology 24, 97-129. https://doi.org/10.1016/S0893-133X(00)00195-0

Koppe, G., Bruckner, G., Hartig, W., Delpech, B., Bigl, V., 1997. Characterization of proteoglycan-containing perineuronal nets by enzymatic treatments of rat brain sections. Histochem. J. 29, 11-20. https://doi.org/10.1023/A:1026408716522

Koss, W.A., Belden, C.E., Hristov, A.D., Juraska, J.M., 2014. Dendritic remodeling in the adolescent medial prefrontal cortex and the basolateral amygdala of male and female rats. Synapse 68, 61-72. https://doi.org/10.1002/syn.21716

Lasek, A.W., Chen, H., Chen, W.-Y., 2017. Releasing Addiction Memories Trapped in Perineuronal Nets. Trends Genet. TIG. https://doi.org/10.1016/j.tig.2017.12.004

LeDoux, J., 2003. The Emotional Brain, Fear, and the Amygdala. Cell. Mol. Neurobiol. 23, 727-738. https://doi.org/10.1023/A:1025048802629 
LeDoux, J.E., 2014. Coming to terms with fear. Proc. Natl. Acad. Sci. U. S. A. 111, 2871-2878. https://doi.org/10.1073/pnas.1400335111

LeDoux, J.E., 2000. Emotion Circuits in the Brain. Annu. Rev. Neurosci. 23, 155-184. https://doi.org/10.1146/annurev.neuro.23.1.155

LeDoux, J.E., Iwata, J., Cicchetti, P., Reis, D.J., 1988. Different projections of the central amygdaloid nucleus mediate autonomic and behavioral correlates of conditioned fear. J. Neurosci. 8, 2517-2529.

Lensjø, K.K., Lepperød, M.E., Dick, G., Hafting, T., Fyhn, M., 2016. Removal of perineuronal nets unlocks juvenile plasticity through network mechanisms of decreased inhibition and increased gamma activity. J. Neurosci. 2504-16. https://doi.org/10.1523/JNEUROSCI.2504-16.2016

Li, Y., Li, Z.-X., Jin, T., Wang, Z.-Y., Zhao, P., 2017. Tau Pathology Promotes the Reorganization of the Extracellular Matrix and Inhibits the Formation of Perineuronal Nets by Regulating the Expression and the Distribution of Hyaluronic Acid Synthases. J. Alzheimers Dis. Preprint, 1-15. https://doi.org/10.3233/JAD-160804

Lin, T.W., Tan, Z., Barik, A., Yin, D.-M., Brudvik, E., Wang, H., Xiong, W.-C., Mei, L., 2018. Regulation of synapse development by Vgat deletion from ErbB4-positive interneurons. J. Neurosci. Off. J. Soc. Neurosci. https://doi.org/10.1523/JNEUROSCI.0669-17.2018

Lopez, J., Herbeaux, K., Cosquer, B., Engeln, M., Muller, C., Lazarus, C., Kelche, C., Bontempi, B., Cassel, J.-C., de Vasconcelos, A.P., 2012. Context-dependent modulation of hippocampal and cortical recruitment during remote spatial memory retrieval. Hippocampus 22, 827-841. https://doi.org/10.1002/hipo.20943

Maren, S., Holmes, A., 2016. Stress and Fear Extinction. Neuropsychopharmacology 41, 58-79. https://doi.org/10.1038/npp.2015.180

Massey, J.M., Hubscher, C.H., Wagoner, M.R., Decker, J.A., Amps, J., Silver, J., Onifer, S.M., 2006. Chondroitinase ABC Digestion of the Perineuronal Net Promotes Functional Collateral Sprouting in the Cuneate Nucleus after Cervical Spinal Cord Injury. J. Neurosci. 26, 4406-4414. https://doi.org/10.1523/JNEUROSCI.5467-05.2006

McCann, R.F., Ross, D.A., 2017. A Fragile Balance: Dendritic Spines, Learning, and Memory. Biol. Psychiatry 82, e11-e13. https://doi.org/10.1016/j.biopsych.2017.05.020

McDonald, A.J., Hamilton, P.G., Barnstable, C.J., 2017. Perineuronal nets labeled by monoclonal antibody VC1.1 ensheath interneurons expressing parvalbumin and calbindin in the rat amygdala. Brain Struct. Funct. 1-16. https://doi.org/10.1007/s00429-017-1542-8

Medina, J.F., Christopher Repa, J., Mauk, M.D., LeDoux, J.E., 2002. Parallels between cerebellum- and amygdala-dependent conditioning. Nat. Rev. Neurosci. 3, 122131. https://doi.org/10.1038/nrn728

Miyata, S., Kitagawa, H., 2016. Chondroitin 6-Sulfation Regulates Perineuronal Net Formation by Controlling the Stability of Aggrecan. Neural Plast. 2016. https://doi.org/10.1155/2016/1305801 
Miyata, S., Komatsu, Y., Yoshimura, Y., Taya, C., Kitagawa, H., 2012. Persistent cortical plasticity by upregulation of chondroitin 6-sulfation. Nat. Neurosci. 15, 414-422. https://doi.org/10.1038/nn.3023

Molchan, S.E., Sunderland, T., Mclntosh, A.R., Herscovitch, P., Schreurs, B.G., 1994. A functional anatomical study of associative learning in humans. Proc. Natl. Acad. Sci. 91, 8122-8126.

Monfils, M.-H., Cowansage, K.K., Klann, E., LeDoux, J.E., 2009. ExtinctionReconsolidation Boundaries: Key to Persistent Attenuation of Fear Memories. Science 324, 951-955. https://doi.org/10.1126/science.1167975

Morris, N.P., Henderson, Z., 2000. Perineuronal nets ensheath fast spiking, parvalbumin-immunoreactive neurons in the medial septum/diagonal band complex. Eur. J. Neurosci. 12, 828-838. https://doi.org/10.1046/j.14609568.2000.00970.x

Mueller, A.L., Davis, A., Sovich, S., Carlson, S.S., Robinson, F.R., 2016. Distribution of $\mathrm{N}-$ Acetylgalactosamine-Positive Perineuronal Nets in the Macaque Brain: Anatomy and Implications. Neural Plast. 2016, 6021428. https://doi.org/10.1155/2016/6021428

Nabel, E.M., Morishita, H., 2013. Regulating Critical Period Plasticity: Insight from the Visual System to Fear Circuitry for Therapeutic Interventions. Front. Psychiatry 4. https://doi.org/10.3389/fpsyt.2013.00146

Nakamura, K., Greenwood, A., Binder, L., Bigio, E.H., Denial, S., Nicholson, L., Zhou, X.Z., Lu, K.P., 2012. Proline isomer-specific antibodies reveal the early pathogenic tau conformation in Alzheimer's disease. Cell 149, 232-244. https://doi.org/10.1016/j.cell.2012.02.016

Narayanan, R., Johnston, D., 2007. Long-Term Potentiation in Rat Hippocampal Neurons Is Accompanied by Spatially Widespread Changes in Intrinsic Oscillatory Dynamics and Excitability. Neuron 56, 1061-1075. https://doi.org/10.1016/j.neuron.2007.10.033

Nguyen, Q., Murphy, G., Hughes, C.E., Mort, J.S., Roughley, P.J., 1993. Matrix metalloproteinases cleave at two distinct sites on human cartilage link protein. Biochem. J. 295 ( Pt 2), 595-598.

Olney, N.T., Spina, S., Miller, B.L., 2017. Frontotemporal Dementia. Neurol. Clin. 35, 339-374. https://doi.org/10.1016/j.ncl.2017.01.008

Passamonti, L., Rodriguez, P.V., Hong, Y.T., Allinson, K.S.J., Williamson, D., Borchert, R.J., Sami, S., Cope, T.E., Bevan-Jones, W.R., Jones, P.S., Arnold, R., Surendranathan, A., Mak, E., Su, L., Fryer, T.D., Aigbirhio, F.I., O’Brien, J.T., Rowe, J.B., 2017. F-18-AV-1451 positron emission tomography in Alzheimer's disease and progressive supranuclear palsy. Brain 140, 781-791. https://doi.org/10.1093/brain/aww340

Pennanen, C., Kivipelto, M., Tuomainen, S., Hartikainen, P., Hänninen, T., Laakso, M.P., Hallikainen, M., Vanhanen, M., Nissinen, A., Helkala, E.-L., Vainio, P., Vanninen, R., Partanen, K., Soininen, H., 2004. Hippocampus and entorhinal cortex in mild cognitive impairment and early AD. Neurobiol. Aging 25, 303-310. https://doi.org/10.1016/S0197-4580(03)00084-8

Petanjek, Z., Judaš, M., Šimić, G., Rašin, M.R., Uylings, H.B.M., Rakic, P., Kostović, I., 2011. Extraordinary neoteny of synaptic spines in the human prefrontal cortex. 
Proc. Natl. Acad. Sci. U. S. A. 108, 13281-13286.

https://doi.org/10.1073/pnas.1105108108

Phillips, R.G., LeDoux, J.E., 1992. Differential contribution of amygdala and hippocampus to cued and contextual fear conditioning. Behav. Neurosci. 106, 274-285. https://doi.org/10.1037/0735-7044.106.2.274

Pignataro, A., Pagano, R., Guarneri, G., Middei, S., Ammassari-Teule, M., 2017. Extracellular matrix controls neuronal features that mediate the persistence of fear. Brain Struct. Funct. 222, 3889-3898. https://doi.org/10.1007/s00429-017$1440-0$

Rescorla, R.A., 2004. Spontaneous Recovery. Learn. Mem. 11, 501-509. https://doi.org/10.1101//m.77504

Riga, D., Kramvis, I., Koskinen, M.K., Bokhoven, P. van, Harst, J.E. van der, Heistek, T.S., Timmerman, A.J., Nierop, P. van, Schors, R.C. van der, Pieneman, A.W., Weger, A. de, Mourik, Y. van, Schoffelmeer, A.N.M., Mansvelder, H.D., Meredith, R.M., Hoogendijk, W.J.G., Smit, A.B., Spijker, S., 2017. Hippocampal extracellular matrix alterations contribute to cognitive impairment associated with a chronic depressive-like state in rats. Sci. Transl. Med. 9, eaai8753. https://doi.org/10.1126/scitransImed.aai8753

Rogers, R.F., Britton, G.B., Steinmetz, J.E., 2001. Learning-related interpositus activity is conserved across species as studied during eyeblink conditioning in the rat. Brain Res. 905, 171-177. https://doi.org/10.1016/S0006-8993(01)02532-X

Rogers, S.L., Rankin-Gee, E., Risbud, R.M., Porter, B.E., Marsh, E.D., 2018. Normal Development of the Perineuronal Net in Humans; In Patients with and without Epilepsy. Neuroscience. https://doi.org/10.1016/j.neuroscience.2018.05.039

Schreurs, B.G., Burhans, L.B., Smith-Bell, C.A., Mrowka, S.W., Wang, D., 2013. Ontogeny of trace eyeblink conditioning to shock-shock pairings in the rat pup. Behav. Neurosci. 127, 114-120. https://doi.org/10.1037/a0031298

Scott, J.C., Matt, G.E., Wrocklage, K.M., Crnich, C., Jordan, J., Southwick, S.M., Krystal, J.H., Schweinsburg, B.C., 2015. A quantitative meta-analysis of neurocognitive functioning in posttraumatic stress disorder. Psychol. Bull. 141, 105-140. https://doi.org/10.1037/a0038039

Selkoe, D.J., Hardy, J., 2016. The amyloid hypothesis of Alzheimer's disease at 25 years. EMBO Mol. Med. 8, 595-608. https://doi.org/10.15252/emmm.201606210

Shankar, G.M., Li, S., Mehta, T.H., Garcia-Munoz, A., Shepardson, N.E., Smith, I., Brett, F.M., Farrell, M.A., Rowan, M.J., Lemere, C.A., Regan, C.M., Walsh, D.M., Sabatini, B.L., Selkoe, D.J., 2008. Amyloid- $\beta$ protein dimers isolated directly from Alzheimer's brains impair synaptic plasticity and memory. Nat. Med. 14, 837842. https://doi.org/10.1038/nm1782

Sillitoe, R.V., Fu, Y., Watson, C., 2012. Chapter 11 - Cerebellum, in: Watson, C., Paxinos, G., Puelles, L. (Eds.), The Mouse Nervous System. Academic Press, San Diego, pp. 360-397. https://doi.org/10.1016/B978-0-12-369497-3.10011-1 Slaker, M., Blacktop, J.M., Sorg, B.A., 2016. Caught in the Net: Perineuronal Nets and Addiction. Neural Plast. 2016, e7538208. https://doi.org/10.1155/2016/7538208

Slaker, M., Churchill, L., Todd, R.P., Blacktop, J.M., Zuloaga, D.G., Raber, J., Darling, R.A., Brown, T.E., Sorg, B.A., 2015. Removal of perineuronal nets in the medial 
prefrontal cortex impairs the acquisition and reconsolidation of a cocaine-induced conditioned place preference memory. J. Neurosci. Off. J. Soc. Neurosci. 35, 4190-4202. https://doi.org/10.1523/JNEUROSCI.3592-14.2015

Slaker, M.L., Jorgensen, E.T., Hegarty, D.M., Liu, X., Kong, Y., Zhang, F., Linhardt, R.J., Brown, T.E., Aicher, S.A., Sorg, B.A., 2018. Cocaine Exposure Modulates Perineuronal Nets and Synaptic Excitability of Fast-Spiking Interneurons in the Medial Prefrontal Cortex. eNeuro 5. https://doi.org/10.1523/ENEURO.022118.2018

Sorg, B.A., Berretta, S., Blacktop, J.M., Fawcett, J.W., Kitagawa, H., Kwok, J.C.F., Miquel, M., 2016. Casting a Wide Net: Role of Perineuronal Nets in Neural Plasticity. J. Neurosci. 36, 11459-11468. https://doi.org/10.1523/JNEUROSCI.2351-16.2016

Stamenkovic, V., Stamenkovic, S., Jaworski, T., Gawlak, M., Jovanovic, M., Jakovcevski, I., Wilczynski, G.M., Kaczmarek, L., Schachner, M., Radenovic, L., Andjus, P.R., 2017. The extracellular matrix glycoprotein tenascin-C and matrix metalloproteinases modify cerebellar structural plasticity by exposure to an enriched environment. Brain Struct. Funct. 222, 393-415.

https://doi.org/10.1007/s00429-016-1224-y

Steinmetz, J.E., Lavond, D.G., Thompson, R.F., 1989. Classical conditioning in rabbits using pontine nucleus stimulation as a conditioned stimulus and inferior olive stimulation as an unconditioned stimulus. Synapse 3, 225-233. https://doi.org/10.1002/syn.890030308

Suttkus, A., Holzer, M., Morawski, M., Arendt, T., 2016. The neuronal extracellular matrix restricts distribution and internalization of aggregated Tau-protein. Neuroscience 313, 225-235. https://doi.org/10.1016/j.neuroscience.2015.11.040

Tewari, B.P., Chaunsali, L., Campbell, S.L., Patel, D.C., Goode, A.E., Sontheimer, H., 2018. Perineuronal nets decrease membrane capacitance of peritumoral fast spiking interneurons in a model of epilepsy. Nat. Commun. 9, 4724. https://doi.org/10.1038/s41467-018-07113-0

Thompson, E.H., Lensjø, K.K., Wigestrand, M.B., Malthe-Sørenssen, A., Hafting, T., Fyhn, M., 2017. Removal of perineuronal nets disrupts recall of a remote fear memory. Proc. Natl. Acad. Sci. 201713530. https://doi.org/10.1073/pnas.1713530115

Thompson, L.T., Moyer, J.R., Disterhoft, J.F., 1996. Transient changes in excitability of rabbit CA3 neurons with a time course appropriate to support memory consolidation. J. Neurophysiol. 76, 1836-1849.

Tsien, R.Y., 2013. Very long-term memories may be stored in the pattern of holes in the perineuronal net. Proc. Natl. Acad. Sci. 110, 12456-12461. https://doi.org/10.1073/pnas.1310158110

Tuna, G., Yener, G.G., Oktay, G., Hüray İşlekel, G., Güldal Kİrkali, F., 2018. Evaluation of Matrix Metalloproteinase-2 (MMP-2) and -9 (MMP-9) and Their Tissue Inhibitors (TIMP-1 and TIMP-2) in Plasma from Patients with Neurodegenerative Dementia. J. Alzheimers Dis. JAD. https://doi.org/10.3233/JAD-180752

Umemori, J., Winkel, F., Castrén, E., Karpova, N.N., 2015. Distinct effects of perinatal exposure to fluoxetine or methylmercury on parvalbumin and perineuronal nets, the markers of critical periods in brain development. Int. J. Dev. Neurosci. Off. J. 
Int. Soc. Dev. Neurosci. 44, 55-64.

https://doi.org/10.1016/j.ijdevneu.2015.05.006

Umemori, J., Winkel, F., Didio, G., Llach Pou, M., Castrén, E., 2018. iPlasticity: induced juvenile-like plasticity in the adult brain as a mechanism of antidepressants.

Psychiatry Clin. Neurosci. https://doi.org/10.1111/pcn.12683

Van den Steen, P.E., Dubois, B., Nelissen, I., Rudd, P.M., Dwek, R.A., Opdenakker, G., 2002. Biochemistry and molecular biology of gelatinase $B$ or matrix metalloproteinase-9 (MMP-9). Crit. Rev. Biochem. Mol. Biol. 37, 375-536. https://doi.org/10.1080/10409230290771546

VanElzakker, M.B., Dahlgren, M.K., Davis, F.C., Dubois, S., Shin, L.M., 2014. From Pavlov to PTSD: the extinction of conditioned fear in rodents, humans, and anxiety disorders. Neurobiol. Learn. Mem. 113, 3-18. https://doi.org/10.1016/j.nlm.2013.11.014

Végh, M.J., Heldring, C.M., Kamphuis, W., Hijazi, S., Timmerman, A.J., Li, K.W., van Nierop, P., Mansvelder, H.D., Hol, E.M., Smit, A.B., van Kesteren, R.E., 2014. Reducing hippocampal extracellular matrix reverses early memory deficits in a mouse model of Alzheimer's disease. Acta Neuropathol. Commun. 2. https://doi.org/10.1186/s40478-014-0076-z

Verret, L., Krezymon, A., Halley, H., Trouche, S., Zerwas, M., Lazouret, M., Lassalle, J.M., Rampon, C., 2013. Transient enriched housing before amyloidosis onset sustains cognitive improvement in Tg2576 mice. Neurobiol. Aging 34, 211-225. https://doi.org/10.1016/j.neurobiolaging.2012.05.013

Wang, D., Fawcett, J., 2012. The perineuronal net and the control of CNS plasticity. Cell Tissue Res. 349, 147-160. https://doi.org/10.1007/s00441-012-1375-y

Wang, D., Schreurs, B.G., 2014. Maturation of membrane properties of neurons in the rat deep cerebellar nuclei. Dev. Neurobiol. 74, 1268-1276. https://doi.org/10.1002/dneu.22203

Wang, D., Schreurs, B.G., 2010. Dietary cholesterol modulates the excitability of rabbit hippocampal CA1 pyramidal neurons. Neurosci. Lett. 479, 327-331. https://doi.org/10.1016/j.neulet.2010.05.090

Wang, D., Smith-Bell, C.A., Burhans, L.B., O'Dell, D.E., Bell, R.W., Schreurs, B.G., 2018. Changes in membrane properties of rat deep cerebellar nuclear projection neurons during acquisition of eyeblink conditioning. Proc. Natl. Acad. Sci. 115, E9419-E9428. https://doi.org/10.1073/pnas.1808539115

Wen, T.H., Afroz, S., Reinhard, S.M., Palacios, A.R., Tapia, K., Binder, D.K., Razak, K.A., Ethell, I.M., 2017. Genetic Reduction of Matrix Metalloproteinase-9 Promotes Formation of Perineuronal Nets Around Parvalbumin-Expressing Interneurons and Normalizes Auditory Cortex Responses in Developing Fmr1 Knock-Out Mice. Cereb. Cortex N. Y. N 1991 1-14. https://doi.org/10.1093/cercor/bhx258

Wesén, E., Gallud, A., Paul, A., Lindberg, D.J., Malmberg, P., Esbjörner, E.K., 2018. Cell surface proteoglycan-mediated uptake and accumulation of the Alzheimer's disease peptide $A \beta(1-42)$. Biochim. Biophys. Acta BBA - Biomembr. 1860, 2204-2214. https://doi.org/10.1016/j.bbamem.2018.08.010

Woodruff-Pak, D.S., 1997. Classical conditioning. Int. Rev. Neurobiol. 41, 341-366. https://doi.org/10.1016/s0074-7742(08)60359-1 
Xue, Y.-X., Xue, L.-F., Liu, J.-F., He, J., Deng, J.-H., Sun, S.-C., Han, H.-B., Luo, Y.-X., Xu, L.-Z., Wu, P., Lu, L., 2014. Depletion of Perineuronal Nets in the Amygdala to Enhance the Erasure of Drug Memories. J. Neurosci. 34, 6647-6658. https://doi.org/10.1523/JNEUROSCI.5390-13.2014

Yang, S., Hilton, S., Alves, J.N., Saksida, L.M., Bussey, T., Matthews, R.T., Kitagawa, H., Spillantini, M.G., Kwok, J.C.F., Fawcett, J.W., 2017. Antibody recognizing 4sulfated chondroitin sulfate proteoglycans restores memory in tauopathy-induced neurodegeneration. Neurobiol. Aging 59, 197-209. https://doi.org/10.1016/j.neurobiolaging.2017.08.002

Yukawa, T., Iwakura, Y., Takei, N., Saito, M., Watanabe, Y., Toyooka, K., Igarashi, M., Niizato, K., Oshima, K., Kunii, Y., Yabe, H., Matsumoto, J., Wada, A., Hino, M., Iritani, S., Niwa, S., Takeuchi, R., Takahashi, H., Kakita, A., Someya, T., Nawa, H., 2018. Pathological alterations of chondroitin sulfate moiety in postmortem hippocampus of patients with schizophrenia. Psychiatry Res. 270, 940-946. https://doi.org/10.1016/j.psychres.2018.10.062

Zhang, Z., Ohtake-Niimi, S., Kadomatsu, K., Uchimura, K., 2016. Reduced molecular size and altered disaccharide composition of cerebral chondroitin sulfate upon Alzheimer's pathogenesis in mice. Nagoya J. Med. Sci. 78, 293-301. 
Chapter 2. The Perineuronal Net and the Cerebellum 


\subsection{The Perineuronal Net is abundant in the cerebellum}

The perineuronal net (PNN) is found in many areas of the central nervous system but the abundance of neurons covered with it varies throughout the brain and spinal cord. Figure 2.1 (preliminary data) shows that in the adult rat brain, there are more $\mathrm{PNN}+$ cells in the deep cerebellar nuclei (DCN) compared to the amygdala, and this is also the case in the monkey brain (Mueller et al., 2016). One of many questions, is how the PNN changes throughout development in the rat anterior interpositus nucleus (AIN), one of the

DCN. Others have

outlined the components

of the PNN in the rat DCN

(Carulli et al., 2006, 2007)

or early timepoints in development (Hirono et al., 2018) but there is no complete study of the development of the PNN in the AIN from the
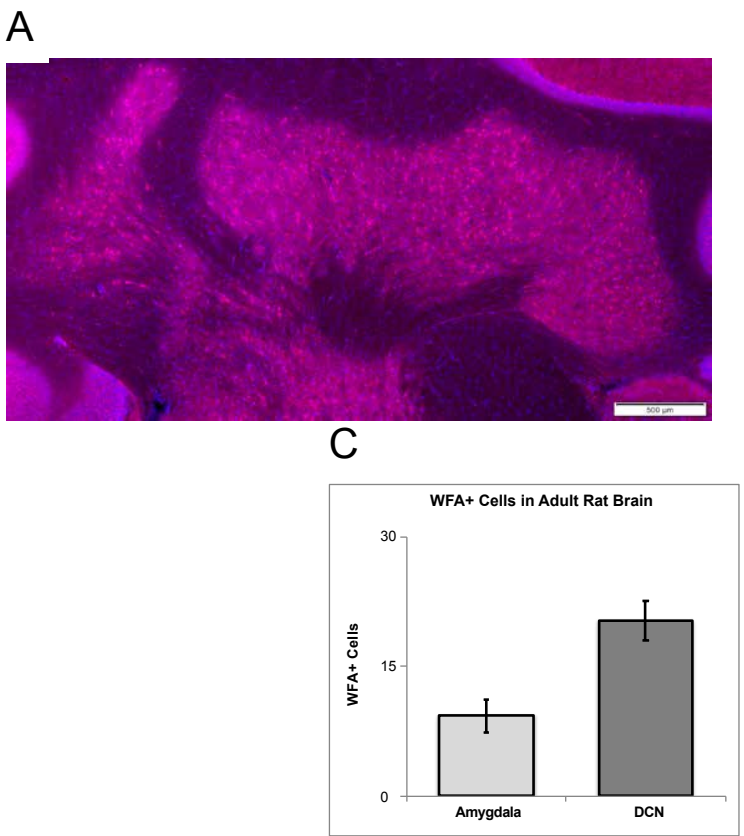

B

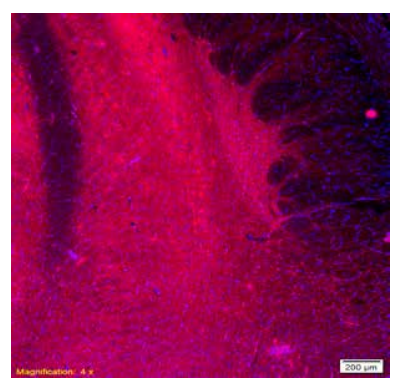

There were less PNN+ cells in the amygdala (A.) than the AIN (B).

Error bars SEM.

the PNN is responsible for

closure of the critical period would be corroborated if the AIN has PNN+ neurons when 
rats are able to learn eyeblink conditioning (EBC). There has been some investigation into modifying the PNN in the DCN or cerebellar cortex (Corvetti and Rossi, 2005; Carulli et al., 2013; de Winter et al., 2016; Carbo-Gas et al., 2017; Stamenkovic et al., 2017). Yet there has been relatively little inquiry into how this affects cerebellar learning and only recently has the function of the PNN in the DCN begun to be investigated (Hirono et al., 2018; Carulli et al., 2020). The low level of PNN studies in the DCN compared to other $\mathrm{PNN}+$ regions is rather surprising since the cerebellum has been implicated in multiple diseases that also are related to the PNN (post-traumatic stress disorder (PTSD), Alzheimer's disease (AD), Autism for example).

\subsection{The Cerebellum is an archetypal system fit for many studies related to the}

\section{PNN}

\subsubsection{Cerebellar learning is a useful way to study memory}

In addition, the DCN, including the AIN, are a site of converging inputs from excitatory and inhibitory cells and provides the only output of the cerebellum; for review of the cerebellum and EBC see Thompson and Steinmetz, 2009, for a historical review of the cerebellum see Glickstein et al., 2009. Figure 2.2 depicts a schematic of the well-studied neural circuitry underlying delay EBC, a paradigm where conditioned stimulus (CS) - unconditioned stimulus (US) pairings coterminate. The cerebellum has two main inputs arising from the brainstem. The climbing fibers from the inferior olive convey information about the US while information about the CS is conveyed along mossy fibers from the pontine nucleus. Stimulation of the mossy and climbing fibers must be separated in time for the animal to learn (Steinmetz et al., 1989); more specifically, the mossy fibers must be stimulated at least 100 milliseconds prior to the climbing fibers. Both fibers can synapse directly onto neurons of the AIN and also synapse onto cells 
within the cerebellar cortex. The conditioned response (CR) pathway originates in the AIN and exits the cerebellum via the superior cerebellar peduncle to synapse at the red nucleus contralateral to the AIN. The red nucleus sends fibers to the motor nucleus (facial nucleus) ipsilateral to the AIN; this is why a lesion of the AIN only affects the CR on the ipsilateral side. The AIN is in

a perfect position anatomically to integrate both the input and output pathways crucial to EBC and therefore has been implicated as the critical site of learning and memory for EBC. In support, lesions to the AIN completely prevent acquisition, expression and savings of the CR whereas

Figure 2.2. shows the Eyeblink conditioning circuit. The Interpositus nuclei is situated perfectly to receive excitatory and inhibitory inputs associated with eyeblink conditioning.

The Eyeblink Conditioning Circuit

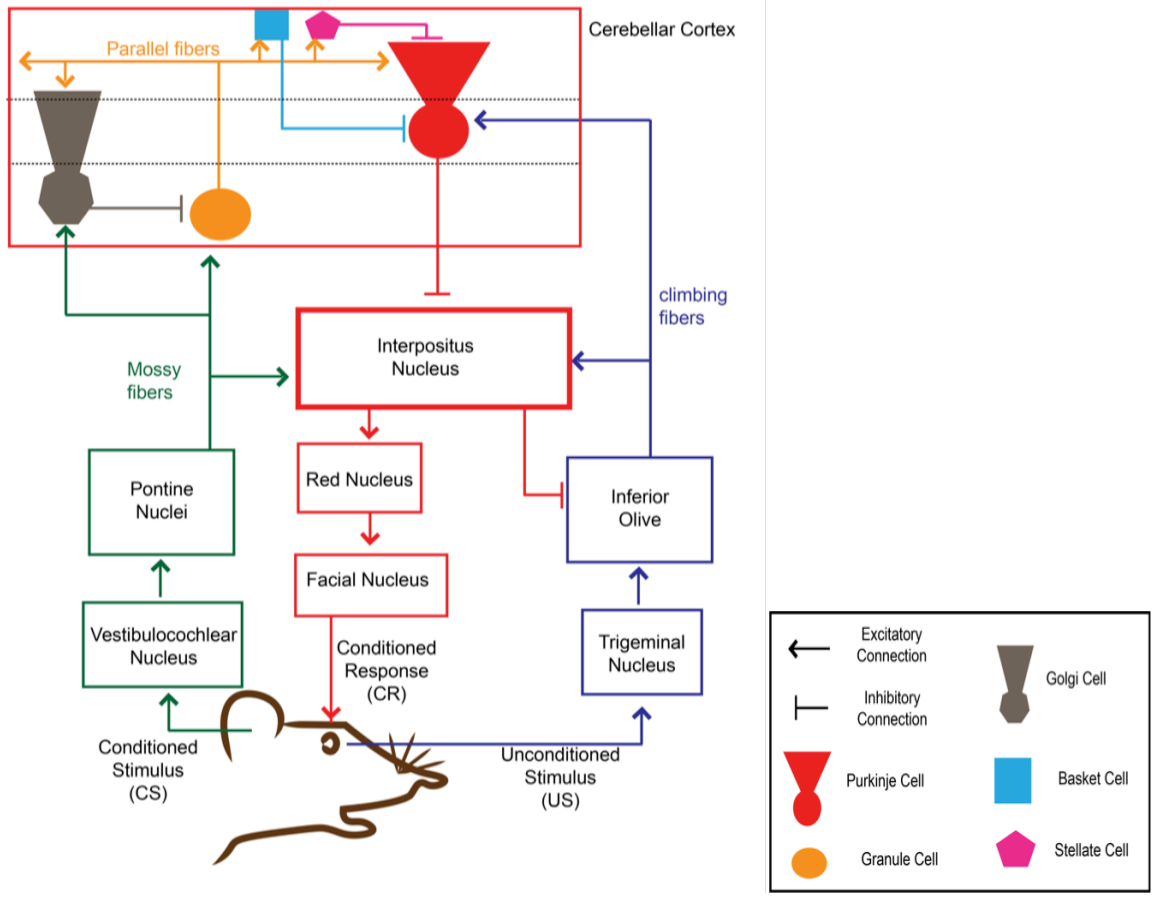

Granule Cell lesions to downstream targets of the AIN involved in the output of EBC such as the red nucleus only eliminate the expression of the CR (Thompson and Steinmetz, 2009).

\subsubsection{The cerebellum is connected to other brain regions that are also PNN+}

The cerebellum has also been found to have indirect connections to regions like the amygdala and hippocampus and is involved in emotional processing as well as 
learning and memory (Medina et al., 2002; Farley et al., 2016, 2018). In adult male rats, bilateral muscimol infusion inactivated the central nucleus of the amygdala and consequently impaired EBC acquisition and retention with a visual CS and EBC acquisition with an auditory CS. These changes were thought to be mediated by connections from the amygdala $\rightarrow$ thalamus $\rightarrow$ pontine nuclei $\rightarrow$ cerebellum (Farley et al., 2016, 2018). These factors render the DCN an interesting area for studying the processes underlying learning and memory and how the PNN may influence cerebellar dependent learning.

There is little information regarding the development of the PNN in the DCN or the cerebellar cortex in young or old animals (Brückner et al., 2000; Carulli et al., 2006). It is currently unknown if there are differences in the number of PNN+ cells or if there are regional differences in the developing $\mathrm{DCN}$ or cerebellar cortex as the animal develops beyond the first few weeks to months after birth. Investigating regions outside of the amygdala and hippocampus is also necessary to understand the PNN's role in aversive memories. Current work shows that manipulating the PNN outside of the amygdala still results in impaired recall of remote fear memories. By degrading the PNN in the secondary visual cortex, theta oscillations between the secondary visual cortex and the amygdala are disrupted and freezing behavior decreases compared to controls (Thompson et al., 2017). These results suggest that an intact PNN in the visual system is necessary for remote fear memories of visual cues. We would expect that enzymatic degradation of the PNN in the auditory cortex may remove fear memory of an auditory CS. The PNN+ in the cerebellum may also have a supporting role in stabilizing fear 
memories since it can modulate emotional related structures including the hippocampus and amygdala (Burhans and Schreurs, 2008; Blatt et al., 2013).

\subsubsection{Development in the cerebellum alters behavior and may be related to the PNN}

The cerebellum is a good model for studying brain development in general due the dynamic and cortical layer specific migration of certain populations of cerebellar cells (Rakic, 1971, 1990; Galas et al., 2017). Gelatinases like MMP-9 are key in directing cerebellar cell morphology. Ribonucleic acid (RNA) analysis shows that MMP-9 levels are elevated in the first 3 postnatal days (P3) in the rat and then sharply declines until a second, small burst appears around P20 (Ayoub et al., 2005). This secondary elevation in MMP-9 RNA is closely timed with the migration of granule cells in the cerebellar cortex, and our own preliminary work with rats shows that around P20 there are significantly more PNN+ cells in the AIN than at earlier postnatal ages such as P12. We also have previous work showing that rat pups do not acquire EBC strongly until P20 (Schreurs et al., 2013), close to the age when the PNN first appears significantly increased in the AIN. Whether these changes in MMP-9 are related to the PNN formation in the cerebellar cortex or DCN has not been explored. Blocking MMP-9 in cerebellar explant cultures has been shown to attenuate the ability of granule cell precursors to migrate. In vivo MMP-9 knock-out mice also have granule cell precursors that fail to reach their final destination in the inner granule layer of the cerebellar cortex (Vaillant et al., 2003). However, no one has investigated changes in MMP-9 protein levels or activity following perturbation of the PNN in adult animals. The cerebellum's role in neural development, specifically the critical period, is being further investigated in relation to 
neurodevelopmental disorders like Autism Spectrum Disorders see Wang et al., 2014

The circuitry involved in EBC must be fairly developed for an animal to actually learn and retain $\mathrm{EBC}$, with the primary rate-limiting factor being development of sensory input pathways to the pontine nucleus. It has been shown that EBC using an auditory CS in rats can occur as early as P17-18 but is much more rapid at P24 (Stanton et al., 1992). As stated earlier, our own

experience with rat preweanlings has shown that reliable levels of conditioning do not emerge until the pup is P18. It is difficult to train rats younger than $\mathrm{P} 17-18$ with an auditory CS due to an immature auditory sensory pathway (Freeman and Rabinak, 2005), but training is possible if the $\mathrm{CS}$ is a somatosensory stimulus like shock (Schreurs et al., 2013) or vibration (Goldsberry et al., 2014), because unlike auditory pathways, the somatosensory pathways matures prenatally. Training rats as young
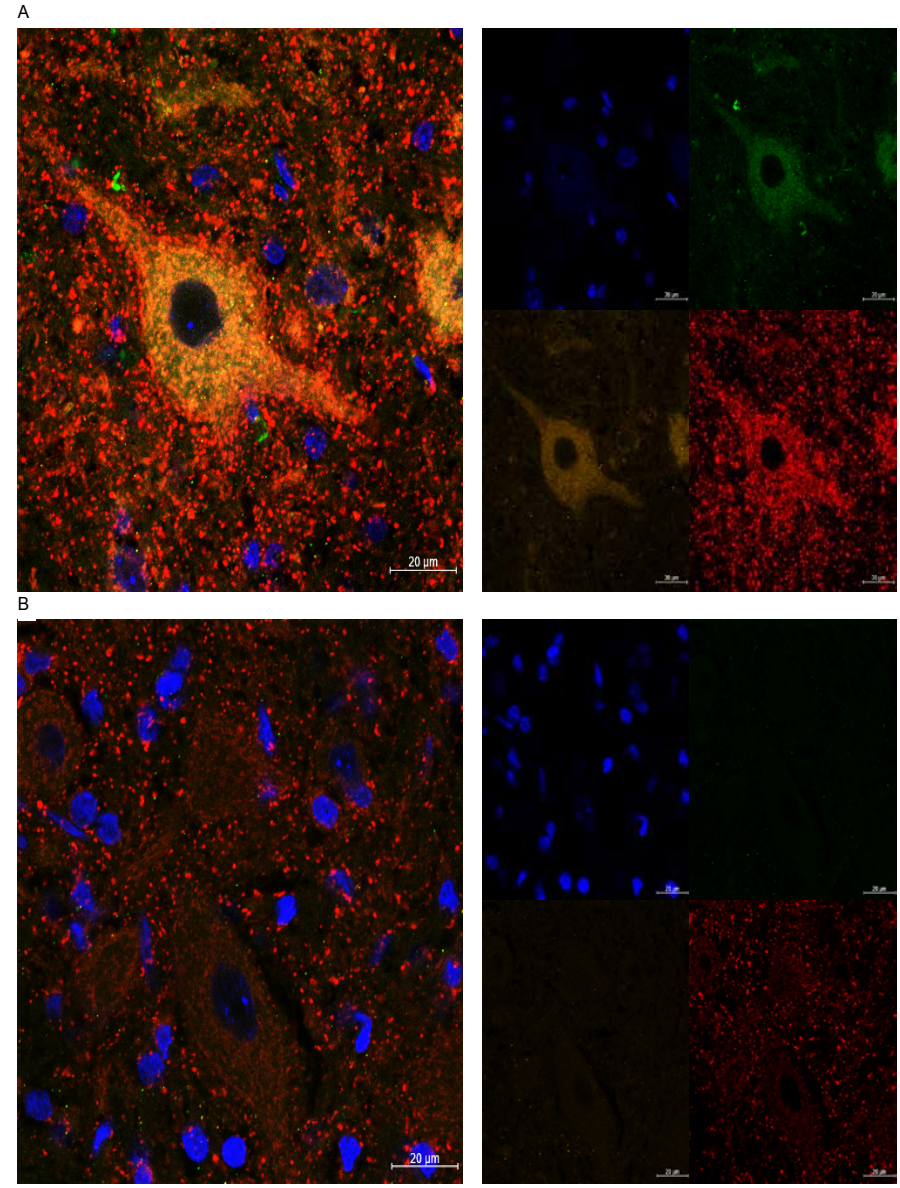

Figure 2.3. WFA and postsynaptic marker labeling in the rat. The figure in panel A shows a PNN+ neuron in the AIN of an adult rat. There is robust labeling of both excitatory (PSD95) and inhibitory (gephyrin) post-synaptic markers. WFA labels a dense PNN as well. The figure in panel $B$ shows a $\mathrm{PNN}+$ in the AIN of a rat at P16. There is little to no labeling of any post-synaptic markers. WFA labeling is present but the PNN appears to be more diffuse than in the adult.

Blue: DAPI, Green:PSD95, Orange: Gephyrin, Red: WFA; $63 x$ obj. on Zeiss 710 
as P12 is possible if the pontine nuclei are directly stimulated as the CS.

There is also evidence for age-related differences in extinction of EBC. Juvenile animals had a faster rate of extinction compared to moderately older (P24) and true adult rats (P60-90) (Brown and Freeman, 2016). The true adults also were the most resistant to extinction compared to the moderately older group. Despite the differences in extinction, all groups successfully learned the EBC. Together, these results suggest that there are age-related differences in an animal's ability to learn EBC and could be dependent on the presence of the PNN in the DCN and other brainstem structures related to EBC.

We noticed potential differences in the post-synaptic machinery of neurons with a PNN in the cerebellar nuclei. Figure 2.3 panel A shows the morphology of the PNN labeled with WFA, excitatory synapses labeled with PSD-95, and inhibitory synapses with gephyrin in the AIN of a naïve adult rat. Figure 2.3 panel B shows the same labeling but in the AIN of a naïve preweanling at P16. In the adult, there is substantially more labeling of both excitatory and inhibitory post-synaptic markers compared to the P16 rat. WFA reactivity in the AIN is present at P16 but the PNN appears more diffuse compared to the dense structure seen in the adult. We found differences in the ontogeny of the PNN in the DCN (see Ch. 3 (O'Dell et al., 2020)). These differences in the PNN of the DCN neurons of an adult and pre-weanling are likely related to the differences observed in cerebellar-dependent learning at these ages.

\subsection{Manipulating the PNN in the Cerebellum will alter behavior}

The two research groups who studied the effect of PNN manipulation on EBC used headfixed, adult male mice and their unconditioned stimulus was air puff for their 
behavioral study. However, they differed on their approach to digesting the PNN in the DCN to study its effect on cerebellar dependent learning. Hirono et al. used chondroitinase $A B C(C h A B C)$ infusion directly to the $D C N$ while Carulli et al., used a lentivirus expressing chondroitinase. They also waited different lengths of time to allow for PNN disruption. Hirono et al., chose to infuse ChABC into the AIN 4 days prior to EBC while the Carulli group allowed for 21 days before beginning acquisition. These variations in approach led to differences in the amount of digestion of the PNN and subtle differences in behavior. The mice in the Carulli study had far less PNN in comparison to the Hirono mice. Both groups found differences in the acquisition of EBC but only the Carulli study observed differences between virally-injected and control mice during extinction.

In addition to behavior, both groups chose to investigate potential changes to the electrophysiology of these cells. The Hirono group found that cerebellar slices exposed to $C h A B C$ showed enhanced gamma-aminobutyric acid (GABA) release from the presynaptic Purkinje cell onto the glutamatergic DCN neurons. The mice also had increased rebound firing in the DCN, which is associated with higher likelihood of inducing changes responsible for cerebellar learning (Pugh and Raman, 2006; Zheng and Raman, 2010, 2011; Person and Raman, 2012). Carulli et al., observed neurons in the DCN with lower spontaneous firing following viral expression of chondroitinase.

We also observed learning-related changes to the PNN in the DCN of rats exposed to EBC and those that were naïve to any behavioral training. The laboratory has previous experience performing EBC experiments in several species, including rats, rabbits, and humans (Molchan et al., 1994; Sparks and Schreurs, 2003; Schreurs et al., 2013) as well 
as electrophysiological experiments in the rat and rabbit cerebellum (Wang and Schreurs, 2006, 2014; Wang et al., 2018). Preliminary experiments have shown that an adult rat was capable of expressing high levels of \%CRs following 5 days of paired EBC that diminished following three days of tone-only extinction. Figure 2.4 shows Mean \% CR (A.) and CR Amplitude (B) and the PNN+ cells of an animals given EBC and a completely naïve animal $(C)$. There appears to be less PNN in the AIN of rats exposed to EBC compared to naïve. These preliminary observations suggests training and extinction of tone-shock

pairings alone

could be capable

of remodeling the

A

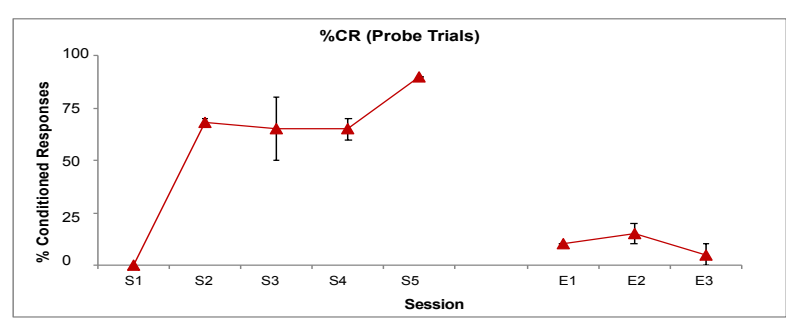

PNN in the AIN.

B

Carulli et al., noticed that

during

acquisition, there

were lower levels

of the PNN in the

mouse AIN but

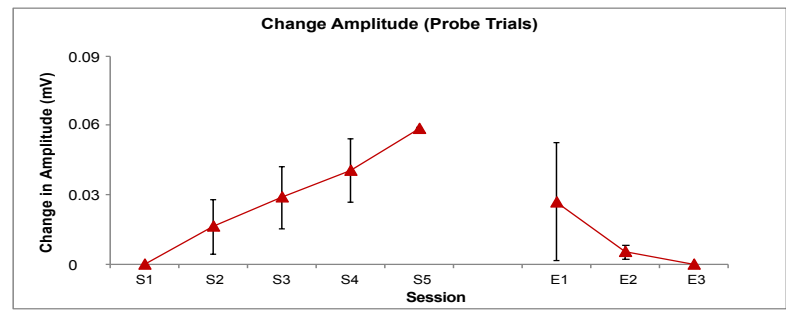

C

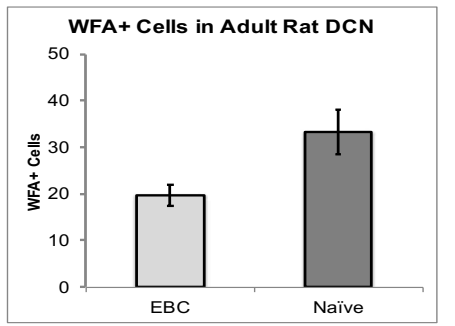

Figure 2.4. EBC acquisition and extinction may alter the PNN+ cells of the DCN.

The graph in A. shows mean \%CRs during EBC and extinction. The rat is at $100 \%$ CR by the end of 5 days of tone-shock pairings. The graph in $B$ shows the mean amplitude of EMG eyelid activity during $E B C$ and $E B C$ extinction. For example, on the $1^{\text {st }}$ day of extinction, the CR amplitude is still high. In C., The graph shows the WFA+ cells in the AIN of a rat exposed to $E B C$ and $E B C$ extinction compared to naïve rats.

Error bars SEM

by the end of the experiment, these levels were closer to the level of naïve and pseudoconditioned controls (Carulli et al., 2020). The daily sessions of tone-shock or tone alone presentations and exposure to these stimuli in the chamber appear to induce proteolytic remodeling of the PNN at synapses to stabilize new memories formed as a 
result of EBC. Further study of the PNN in the cerebellum may better define this relationship. 


\section{References:}

Ayoub AE, Cai T-Q, Kaplan RA, Luo J (2005) Developmental expression of matrix metalloproteinases 2 and 9 and their potential role in the histogenesis of the cerebellar cortex. J Comp Neurol 481:403-415.

Blatt GJ, Oblak AL, Schmahmann JD (2013) Cerebellar Connections with Limbic Circuits: Anatomy and Functional Implications. In: Handbook of the Cerebellum and Cerebellar Disorders, pp 479-496. Springer, Dordrecht. Available at: https://link.springer.com/referenceworkentry/10.1007/978-94-007-1333-8_22 [Accessed January 2, 2018].

Brown KL, Freeman JH (2016) Retention of eyeblink conditioning in periweanling and adult rats. Dev Psychobiol 58:1055-1065.

Brückner G, Grosche J, Schmidt S, Härtig W, Margolis RU, Delpech B, Seidenbecher $\mathrm{Cl}$, Czaniera R, Schachner M (2000) Postnatal development of perineuronal nets in wild-type mice and in a mutant deficient in tenascin-R. J Comp Neurol 428:616-629.

Burhans LB, Schreurs BG (2008) Inactivation of the central nucleus of the amygdala abolishes conditioning-specific reflex modification of the rabbit (Oryctolagus cuniculus) nictitating membrane response and delays classical conditioning. Behav Neurosci 122:75-88.

Carbo-Gas M, Moreno-Rius J, Guarque-Chabrera J, Vazquez-Sanroman D, Gil-Miravet I, Carulli D, Hoebeek F, De Zeeuw C, Sanchis-Segura C, Miquel M (2017) Cerebellar perineuronal nets in cocaine-induced pavlovian memory: Site matters. Neuropharmacology 125:166-180.

Carulli D, Broersen R, de Winter F, Muir EM, Mešković M, de Waal M, de Vries S, Boele $\mathrm{H}-\mathrm{J}$, Canto CB, De Zeeuw Cl, Verhaagen J (2020) Cerebellar plasticity and associative memories are controlled by perineuronal nets. Proc Natl Acad Sci U $S A$.

Carulli D, Foscarin S, Faralli A, Pajaj E, Rossi F (2013) Modulation of semaphorin3A in perineuronal nets during structural plasticity in the adult cerebellum. Mol Cell Neurosci 57:10-22.

Carulli D, Rhodes KE, Brown DJ, Bonnert TP, Pollack SJ, Oliver K, Strata P, Fawcett JW (2006) Composition of perineuronal nets in the adult rat cerebellum and the cellular origin of their components. J Comp Neurol 494:559-577.

Carulli D, Rhodes KE, Fawcett JW (2007) Upregulation of aggrecan, link protein 1, and hyaluronan synthases during formation of perineuronal nets in the rat cerebellum. J Comp Neurol 501:83-94.

Corvetti L, Rossi F (2005) Degradation of chondroitin sulfate proteoglycans induces sprouting of intact purkinje axons in the cerebellum of the adult rat. J Neurosci Off J Soc Neurosci 25:7150-7158.

de Winter F, Kwok JCF, Fawcett JW, Vo TT, Carulli D, Verhaagen J (2016) The Chemorepulsive Protein Semaphorin 3A and Perineuronal Net-Mediated Plasticity. Neural Plast 2016:e3679545.

Farley SJ, Albazboz H, De Corte BJ, Radley JJ, Freeman JH (2018) Amygdala central nucleus modulation of cerebellar learning with a visual conditioned stimulus. Neurobiol Learn Mem 150:84-92. 
Farley SJ, Radley JJ, Freeman JH (2016) Amygdala Modulation of Cerebellar Learning. J Neurosci 36:2190-2201.

Freeman JH, Rabinak CA (2005) Eyeblink conditioning in rats using pontine stimulation as a conditioned stimulus. Integr Physiol Behav Sci 39:180-191.

Galas L, Bénard M, Lebon A, Komuro Y, Schapman D, Vaudry H, Vaudry D, Komuro H (2017) Postnatal Migration of Cerebellar Interneurons. Brain Sci 7:62.

Glickstein M, Strata P, Voogd J (2009) Cerebellum: history. Neuroscience 162:549-559.

Goldsberry ME, Elkin ME, Freeman JH (2014) Sensory system development influences the ontogeny of eyeblink conditioning. Dev Psychobiol 56:1244-1251.

Hirono M, Watanabe S, Karube F, Fujiyama F, Kawahara S, Nagao S, Yanagawa Y, Misonou $\mathrm{H}$ (2018) Perineuronal nets in the deep cerebellar nuclei regulate GABAergic transmission and delay eyeblink conditioning. J Neurosci:3238-17.

Medina JF, Christopher Repa J, Mauk MD, LeDoux JE (2002) Parallels between cerebellum- and amygdala-dependent conditioning. Nat Rev Neurosci 3:122131.

Molchan SE, Sunderland T, McIntosh AR, Herscovitch P, Schreurs BG (1994) A functional anatomical study of associative learning in humans. Proc Natl Acad Sci 91:8122-8126.

Mueller AL, Davis A, Sovich S, Carlson SS, Robinson FR (2016) Distribution of NAcetylgalactosamine-Positive Perineuronal Nets in the Macaque Brain: Anatomy and Implications. Neural Plast 2016:6021428.

O'Dell DE, Schreurs BG, Smith-Bell C, Wang D (2020) Disruption of rat deep cerebellar perineuronal net alters eyeblink conditioning and neuronal electrophysiology. Neurobiol Learn Mem 177:107358.

Person AL, Raman IM (2012) Synchrony and neural coding in cerebellar circuits. Front Neural Circuits 6:97.

Pugh JR, Raman IM (2006) Potentiation of mossy fiber EPSCs in the cerebellar nuclei by NMDA receptor activation followed by postinhibitory rebound current. Neuron 51:113-123.

Rakic $P$ (1971) Neuron-glia relationship during granule cell migration in developing cerebellar cortex. A Golgi and electonmicroscopic study in Macacus rhesus. J Comp Neurol 141:283-312.

Rakic P (1990) Principles of neural cell migration. Experientia 46:882-891.

Schreurs BG, Burhans LB, Smith-Bell CA, Mrowka SW, Wang D (2013) Ontogeny of trace eyeblink conditioning to shock-shock pairings in the rat pup. Behav Neurosci 127:114-120.

Sparks DL, Schreurs BG (2003) Trace amounts of copper in water induce beta-amyloid plaques and learning deficits in a rabbit model of Alzheimer's disease. Proc Natl Acad Sci U S A 100:11065-11069.

Stamenkovic V, Stamenkovic S, Jaworski T, Gawlak M, Jovanovic M, Jakovcevski I, Wilczynski GM, Kaczmarek L, Schachner M, Radenovic L, Andjus PR (2017) The extracellular matrix glycoprotein tenascin- $C$ and matrix metalloproteinases modify cerebellar structural plasticity by exposure to an enriched environment. Brain Struct Funct 222:393-415.

Stanton ME, Freeman JH, Skelton RW (1992) Eyeblink conditioning in the developing rat. Behav Neurosci 106:657-665. 
Steinmetz JE, Lavond DG, Thompson RF (1989) Classical conditioning in rabbits using pontine nucleus stimulation as a conditioned stimulus and inferior olive stimulation as an unconditioned stimulus. Synapse 3:225-233.

Thompson EH, Lensjø KK, Wigestrand MB, Malthe-Sørenssen A, Hafting T, Fyhn M (2017) Removal of perineuronal nets disrupts recall of a remote fear memory. Proc Natl Acad Sci:201713530.

Thompson RF, Steinmetz JE (2009) The role of the cerebellum in classical conditioning of discrete behavioral responses. Neuroscience 162:732-755.

Vaillant C, Meissirel C, Mutin M, Belin M-F, Lund LR, Thomasset N (2003) MMP-9 deficiency affects axonal outgrowth, migration, and apoptosis in the developing cerebellum. Mol Cell Neurosci 24:395-408.

Wang D, Schreurs BG (2006) Characteristics of IA currents in adult rabbit cerebellar Purkinje cells. Brain Res 1096:85-96.

Wang D, Schreurs BG (2014) Maturation of membrane properties of neurons in the rat deep cerebellar nuclei. Dev Neurobiol 74:1268-1276.

Wang D, Smith-Bell CA, Burhans LB, O’Dell DE, Bell RW, Schreurs BG (2018) Changes in membrane properties of rat deep cerebellar nuclear projection neurons during acquisition of eyeblink conditioning. Proc Natl Acad Sci 115:E9419-E9428.

Wang SS-H, Kloth AD, Badura A (2014) The Cerebellum, Sensitive Periods, and Autism. Neuron 83:518-532.

Zheng N, Raman IM (2010) Synaptic inhibition, excitation, and plasticity in neurons of the cerebellar nuclei. Cerebellum Lond Engl 9:56-66.

Zheng N, Raman IM (2011) Prolonged Postinhibitory Rebound Firing in the Cerebellar Nuclei Mediated by Group I Metabotropic Glutamate Receptor Potentiation of LType Calcium Currents. J Neurosci 31:10283-10292. 
Chapter 3. Disruption of Rat Deep Cerebellar Perineuronal Net Alters Eyeblink Conditioning and Neuronal Physiology

This work was submitted and accepted as a publication in volume 177 of the Journal of Neurobiology of Learning and Memory, doi.org/10.1016/j.nlm.2020.107358 


\section{Abstract}

The perineuronal net (PNN) is a specialized type of extracellular matrix found in the central nervous system. The PNN forms on fast spiking neurons during postnatal development but the ontogeny of PNN development has yet to be elucidated. By studying the development and prevalence of the PNN in the juvenile and adult rat brain, we may be able to understand the PNN's role in development and learning and memory. We show that the PNN is fully developed in the deep cerebellar nuclei (DCN) of rats by P18. By using enzymatic digestion of the PNN with chondroitinase $A B C(C h A B C)$, we are able to study how digestion of the PNN affects cerebellar-dependent eyeblink conditioning in vivo and perform electrophysiological recordings from DCN neurons in vitro. In vivo degradation of the PNN resulted in significant differences in eyeblink conditioning amplitude and area. Female animals in the vehicle group demonstrated higher levels of conditioning as well as significantly higher post-probe conditioned responses compared to males in that group, differences not present in the ChABC group. In vitro, we found that DCN neurons with a disrupted PNN following exposure to ChABC had altered membrane properties, fewer rebound spikes, and decreased intrinsic excitability. Together, this study further elucidates the role of the PNN in cerebellar learning in the DCN and is the first to demonstrate PNN degradation may erase sex differences in delay conditioning. Six keywords: PNN, DCN, electrophysiology, eyeblink conditioning, ChABC 


\subsection{Introduction}

Nervous system function is centered on neurons, but these neurons are modulated by many external factors including other cells like glia and even the extracellular matrix (ECM) (Araque et al., 1999; Brückner et al., 1993; Cope \& Gould, 2019; Ferrer-Ferrer \& Dityatev, 2018; Perea et al., 2009; Rakic, 1971). Although there are multiple types of ECM, a specialized ECM structure called the perineuronal net (PNN) plays a key role in myriad functions in the central nervous system (CNS) ranging from memory storage to inhibiting neuronal regeneration following injury (Carulli et al., 2010; Celio et al., 1998; Chu et al., 2018; De Luca \& Papa, 2016; Iwata et al., 1993; Kecskes et al., 2015; Krishnaswamy et al., 2019; Minta et al., 2019; Pizzorusso et al., 2002; Rowlands et al., 2018; Stryker et al., 2017; Suttkus et al., 2016). The PNN can restrict neuronal plasticity by acting as a physical barrier to sprouting neurons, acting as a scaffold for inhibitory molecules, as well as acting as a corral for receptors, like AMPA receptors, at the synapse (for review see Sorg et al., 2016. The PNN is implicated in the exit from a critical period, a period of enhanced experience-dependent plasticity found in sensory processes like audition and vision as well as cognitive processes such as learning and memory (Alberini \& Travaglia, 2017; Hensch, 2005; Hou et al., 2017; Nabel \& Morishita, 2013; Umemori et al., 2015).

The PNN is typically associated with the soma and proximal dendrites of neurons (Brückner et al., 2006; Dityatev et al., 2007; Fawcett et al., 2019; Giamanco et al., 2010; Matthews et al., 2002) and comprises multiple components including hyaluronic acid (HA), chondroitin sulfate proteoglycans (CSPG), hyaluronan and proteoglycan link proteins including brain associated link protein 2 (Bral2) (Bekku et al., 2012; Carulli et al., 2006; Cicanic et al., 2017; Edamatsu et al., 2018) or cartilage link protein 1 (Crtl1) (Carulli et al., 2007; Galtrey et al., 2008) and tenascin-R. Wisteria Floribunda agglutinin (WFA) or lectin is a commonly used marker for PNN because it exclusively binds to residues found in CSPG (Brückner et al., 1996; Hilbig et al., 2001; O'Connor et al., 2019). Together the components assemble into the PNN to ensheathe primarily fast-spiking neurons in many brain regions and across many species. Although 
interneurons, particularly inhibitory parvalbumin-positive interneurons, are more commonly found with a PNN (Baker et al., 2017; Bradshaw et al., 2018; Cabungcal et al., 2013; Guirado et al., 2014; McDonald et al., 2017; Morris \& Henderson, 2000; Ohira et al., 2013; Slaker et al., 2018), there is also a subset of excitatory neurons in the CNS with a PNN (Carstens et al., 2016; Carulli et al., 2007; Morikawa et al., 2017; Seeger et al., 1994). Specifically, the deep cerebellar nuclei (DCN) are enriched with an abundance of large excitatory cells with a PNN (Bekku et al., 2012; Carbo-Gas et al., 2017; Carulli et al., 2006, 2013; de Winter et al., 2016; Edamatsu et al., 2018; Hirono et al., 2018; Mueller et al., 2016; Stamenkovic et al., 2017).

The DCN comprise the sole output of the cerebellum (Glickstein et al., 2009; Thompson \& Steinmetz, 2009). The DCN, including the anterior interpositus nucleus (AIN), receive converging inputs from inhibitory Purkinje cells in the cerebellar cortex and excitatory projections from the mossy fibers originating in the pontine nucleus and the climbing fibers from the inferior olive (Steinmetz et al., 1989). As a result, the DCN are in a perfect position anatomically to integrate both the input and output pathways essential to eyeblink conditioning (EBC) and have been implicated as the crucial site of learning and memory for EBC, particularly the AIN. Although this is a contested view, lesions to the AIN do prevent acquisition, expression, and savings of the eyeblink conditioned response (CR) whereas lesions to downstream targets of the AIN involved in the output of EBC, such as the red nucleus, only eliminate the expression of the CR (Brown \& Woodruff-Pak, 2011; Freeman et al., 1995; Nolan et al., 2002; Thompson \& Steinmetz, 2009). Despite the abundance of the PNN in the DCN, their role in learning and memory following enzymatic disruption has not been as well studied in comparison to disruption of PNN-associated neurons in other brain regions including the hippocampus, amygdala, and regions of the cortex (Chu et al., 2018; Hettiaratchi et al., 2019; McDonald et al., 2017; Sun et al., 2018; Xue et al., 2014).

Although there have been investigations into modifying the PNN in the DCN or cerebellar cortex, there has been little inquiry into how this affects cerebellar learning (Carbo-Gas et al., 
2017; Carulli et al., 2013; Corvetti \& Rossi, 2005; de Winter et al., 2016; Stamenkovic et al., 2017) or how the PNN in the DCN changes throughout development. In fact, there have been only two studies investigating PNN disruption in the DCN and the effects on EBC, and both studied mice with air puff as the unconditioned stimulus. The first study (Hirono et al., 2018) examined EBC and the synaptic electrophysiology of the large excitatory neurons in the DCN following exposure to chondroitinase $A B C(C h A B C)$, a bacterial enzyme that digests the CSPG in the PNN as evidenced by decreased WFA labelling (Bradbury et al., 2002; Brückner et al., 1998; Gogolla et al., 2009; Massey et al., 2006). The second study investigated the effects of injecting a virus expressing chondroitinase into the AIN (Carulli et al., 2020). In the Hirono et al. study, naïve male mice between P21-P23 were given ChABC infusion into the DCN and euthanized 4-6 days later to examine the electrophysiology of DCN neurons following PNN digestion in acute cerebellar slices while Carulli et al., performed in vivo recordings in adult male mice injected with a virus expressing chondroitinase. In both studies, mice that had a reduction of PNN had an enhancement of learning expressed as percent conditioned responses (\%CRs) during acquisition of $E B C$, findings that are unlike the effects of $C h A B C$ digestion in the amygdala, which aided the removal of aversive memories following extinction without altering acquisition (Gogolla et al., 2009). The study by Carulli et al., saw similar group differences during extinction, animals with a digested PNN had lower \%CRs compared to the vehicle controls, as well as group differences during acquisition. Animals in the control group in both the Hirono and Carulli studies had relatively low acquisition \%CRs (around 60\%) even after extensive training compared to other EBC studies using mice (Heiney et al., 2014; Koekkoek et al., 2002). There was no report of additional eyeblink parameters, such as peak latency or response amplitude, which can give a more nuanced assessment of learning effects and are perturbed in patients with diagnoses like post-traumatic stress disorder (Ayers et al., 2003). Carulli et al., found DCN neurons had a lower spontaneous firing rate after exposure to viral expression of chondroitinase (Carulli et al., 2020). Hirono et al., observed PNN disruption by infusion of ChABC in mouse cerebellar slices which 
showed increased amplitude of evoked inhibitory postsynaptic currents (IPSCs) as well as miniinhibitory postsynaptic potential frequency; they also observed loss of the PNN facilitated spontaneous ISPCs (Hirono et al., 2018).

Additional studies of the role of the PNN in EBC are needed to compare differences between species. The other studies only used male mice and, as a result, were unable to assess potential sex differences. Further, cells in the DCN are excitatory neurons with a PNN, which is rather uncommon compared to the vast majority of cells with a PNN. The most common studies of the PNN investigate the relationship between the PNN and inhibitory interneurons. Only a fraction of PNN research includes excitatory cells, suggesting there is a need to look outside the interneuron population. Since EBC is not acquired well prior to P17 (Stanton et al., 1992), it is also in our interest to study how the PNN changes from the preweanling to adult in the rat DCN. The goal of this research was to study the role of the PNN in the DCN of male and female rats by temporarily degrading the PNN with $\mathrm{ChABC}$ in vivo to study how ChABC infusion alters $\mathrm{EBC}$ in adult animals as well as in vitro application to investigate changes in the electrophysiology of the large excitatory neurons of the DCN. Our perturbations of the PNN revealed significant group differences in CR amplitude and area in addition to decreased membrane excitability in large DCN neurons. 


\subsection{Methods:}

\subsubsection{Animals}

Forty-three Long Evans rats (Rattus norvegicus) (male and female) between post-natal day 12 (P12) and 6 months of age were supplied by Charles River (Wilmington, MA). Twenty-six rats underwent surgery, twelve were used for ontogeny analysis, and five were slated for electrophysiological studies. Rats were housed with littermates of the same sex after weaning and were given ad libitum access to food and water and maintained on a $12 \mathrm{~h}$ light/dark cycle, all in accordance with the National Institute of Health guidelines. Adult rats undergoing cannulae implantation and/ or electromyogram (EMG) surgery were housed individually post-surgery. This was to prevent damage to the EMG and/or cannulae via social grooming. All animals were socially housed until they were at least P80. All procedures were approved by the West Virginia University (WVU) Animal Care and Use Committee and the WVU Biosafety Committee.

\subsubsection{Surgeries}

Twenty-six 3-month old rats (13 F, $13 \mathrm{M})$ underwent cannula implantation in the AIN. Eight underwent bilateral cannulae implantation while eighteen had cannula implanted only in the left AIN and were also fitted with the EMG hardware for eyeblink conditioning. Sterile surgical technique was used to implant cannulae into the rat's brain at coordinates determined from the literature with reference to a stereotaxic rat brain atlas (Paxinos \& Watson, 2004). Each rat was sedated with isoflurane ( $5 \%$ induction, $2 \%$ maintenance) with supplemental oxygen $(0.5-1 \mathrm{~L} / \mathrm{min})$. The incision site (scalp) was shaved and washed three times with alternating swipes of Povidoneiodine scrub and $70 \%$ alcohol followed by application of Povidone-iodine solution after which the rat was positioned in a rodent adult stereotaxic (Kopf Model 900LS) device. Bupivacaine (max dose $2 \mathrm{mg} / \mathrm{kg}$ ), a local anesthetic, was infiltrated into the scalp, which was incised with a surgical scalpel blade, to create an approximately $20-\mathrm{mm}$ incision, just rostral to the eyes and just caudal to the ears. The scalp was reflected and cleaned, exposing just enough of the skull to 
accommodate a plastic connector for the EMG implant and up to two guide cannulae. Holes were drilled into the skull for skull screws and guide cannula implantation. Gelfoam (Capital Wholesale Drug Company, 245780) was used to stop any bleeding while drilling as the cannula drill site was over a major vessel. Each guide cannula (C317G/SPC GUIDE 23GA 38172, Plastics One, Roanoke, VA) was attached to the stereotaxic apparatus and slowly lowered at the following stereotaxic coordinates from Bregma AP: $-11.5, \mathrm{ML}:( \pm) 3.8, \mathrm{DV}:-5.2$ from skull surface and then was secured to the skull screws with acrylic dental cement. Following surgery and excepting during infusion, dummy cannulae (C317DC/SPC DUMMY(SM) .010/25MM) were placed into the guide cannulae to protect the opening.

The rats assigned to EBC were next fitted with differential EMG electrodes that were implanted in the left eyelid muscle by inserting a sterile 25-gauge needle (affixed to a 1-mm empty sterile syringe) through the eyelid until the needle tip was visible on the other side as a result of the open scalp incision. The needle was then threaded with the fine wire EMG electrodes and pulled through the eyelid, leaving the recording electrode in the middle of the eyelid and the reference wire in the rostral corner of the eyelid. Where in contact around the eyelid, wires were stripped of insulation and excess wire was trimmed in an effort to prevent displacement via grooming. A bipolar stimulating electrode for delivering the electrical stimulation unconditioned stimulus was implanted subdermally, immediately caudal to the eye. The EMG recording electrode and stimulating electrode wire leads terminated on gold pins in a plastic connector, which was secured to the top of the skull, cannula, and the skull screws with acrylic dental cement. The surgical site was closed with nylon suture on both sides of the plastic connector and/ or cannulae. A nonsteroidal anti-inflammatory analgesic (ketoprofen $1 \mathrm{mg} / \mathrm{mL}$ ) was administered at the end of surgery and $3 \mathrm{~mL}$ of lactated Ringer's solution was given subcutaneously to restore any fluids lost during surgery.

\subsubsection{Eyeblink Conditioning}


Rats were allowed to recover from cannula and/ or EMG surgery for four days and then underwent one session per day for five days of paired tone conditioned stimulus (CS) and periorbital electrical stimulation unconditioned stimulus (US) training followed by one session per day for three days of CS-alone extinction. The rat head plug was connected to a cable connected to a freely-rotating commutator which allowed the rat to move freely around a Coulburn Instruments modular testing cage within a sound-attenuating training chamber. The back wall of the chamber had a panel containing a speaker mounted at a $45^{\circ}$ angle above the testing cage. The cable separated and terminated as the input to an AC/DC differential EMG amplifier (A-M Systems, Sequim, WA) and the output of a stimulus isolator (World Precision Instruments (WPI), Sarasota, FL) for shock delivery. The training chamber was lit with an LED and contained a low-light camera to allow videographic monitoring of the animal at all times. Shock was delivered by a rechargeable, constant-current stimulator (WPI, Model A365) that had been calibrated with a multimeter (WPI). LabVIEW software (National Instruments, Austin, TX) controlled the delivery of stimuli and the recording of eyelid EMG activity. Each adult rat was adapted to the enclosure without stimulus presentations for 10 min before every session. Paired delay conditioning sessions consisted of 100 trials each with 90 paired presentations of a tone CS and a periorbital electrical stimulation US as well as 10 tone-alone test trials or probes presented after every ninth paired trial to assess integrated EMG activity without a shock artifact. The tone CS consisted of a 380-ms, 88-db, 2.8$\mathrm{kHz}$ pure tone. The electrical stimulation US consisted of a $100-\mathrm{ms}, 3.0-4.0-\mathrm{mA}, 60-\mathrm{Hz}, \pm 50-\mathrm{volt}$, square-wave constant-current pulse to the periorbital region using the pre-calibrated stimulator. A blanking circuit in operation during the US prevented the shock from swamping the EMG signal. During the tone-shock paired trials, the CS co-terminated with the US. During acquisition, all trials were separated by an inter-trial interval averaging $30 \mathrm{~s}$. To assess memory of and to extinguish responding to the tone CS, we performed CS-alone extinction beginning one day after the last paired training session. The tone CS was presented by itself for 100 trials daily on each of three consecutive days. Every $10^{\text {th }}$ trial during extinction was considered a probe trial. 


\subsubsection{Behavior Data Analysis}

Data analyses have been described previously (Schreurs et al., 2013; Smith-Bell \& Schreurs, 2017; Wang et al., 2018). In brief, EMG signal was filtered (300 - 3,000-Hz), amplified, and stored (raw EMG), in addition to being rectified and integrated (20-ms time constant). Baseline activity was averaged during the first $200-\mathrm{ms}$ from trial onset. If EMG activity $100-\mathrm{ms}$ before CS onset was four standard deviations (SDs) or more above baseline, the trial was omitted from analysis to ensure that movement or spontaneous blinking artifacts did not artificially inflate response levels ( $\sim 1 \%$ of total trials). During paired trials, we characterized unconditioned responses (URs) to the US as integrated EMG activity occurring 35-ms after the end of the US that exceeded the average baseline value by eight SDs. CRs were assessed as EMG activity that exceeded 8 SDs above the baseline during the period 80 -ms after tone onset until just before US onset. This window ensured that US signal and movement or startle artifacts did not artificially inflate levels of responding. During probe trials, CRs were assessed as EMG activity beginning 80-ms after CS onset, to eliminate the potential for including alpha responses, that was eight SDs above the average baseline value during a $200-\mathrm{ms}$ pre-CS period. In addition to conditioned response frequency indicated as \%CRs, the amplitude of each response was calculated as the average EMG signal during the baseline period, subtracted from the maximum EMG signal during the response period. Area under the curve of the CR was obtained by summing the average heights of 2.5-ms bins of data that occurred during the CR period. Peak latency of the CR was calculated as the time when the maximum CR period height occurred (Schreurs et al., 2013; Wang et al., 2018). If an animal did not reach $70 \%$ CRs during probe trials on at least one day of acquisition, they were excluded from behavioral analyses. This is the laboratory's standard for determining adequate levels of EBC in rats for a determination of conditioning-specific changes in unconditioned responding. Traces were made using OriginPro 2019b (OriginLab ver.9.6.5169 (Academic)). 


\subsubsection{Slice preparation and patch-clamp recordings:}

Procedures identical to those previously published (Wang et al., 2018; Wang \& Schreurs, 2010, 2014; Wang \& Zheng, 2015) were used for slice preparation, electrophysiological recordings, and data analysis. Briefly, P24-28 rats ( $\mathrm{n}=5$ rats) were anesthetized with carbon dioxide and then decapitated. Animals were selected at this age because successful recordings of DCN becomes increasingly difficult after P32 (Wang et al., 2018). After rapid brain removal, coronal cerebellar slices from the cerebellum were cut at $34^{\circ} \mathrm{C}$ on a vibrating slicer (LEICA VT1200S) with sucrose artificial cerebrospinal fluid (ACSF) containing (in $\mathrm{mM}$ ) Sucrose $200, \mathrm{KCl} 2.5, \mathrm{MgCl}_{2} 1.2, \mathrm{CaCl}_{2}$ 0.5, $\mathrm{NaH}_{2} \mathrm{PO}_{4} 1.25, \mathrm{NaHCO}_{3} 26$ and Dextrose 20, incubated for 1 hour at $34{ }^{\circ} \mathrm{C}$ in $95 \% \mathrm{O}_{2}$ - and $5 \% \mathrm{CO}_{2}$-saturated ACSF containing (in mM) NaCl 125, $\mathrm{KCl} 3.0, \mathrm{MgSO}_{4} 1.2, \mathrm{CaCl}_{2} 2.0, \mathrm{NaH}_{2} \mathrm{PO}_{4}$ 1.2, $\mathrm{NaHCO}_{3} 26$ and Dextrose 10. After post-slicing recovery, slices were maintained at room temperature until electrophysiological recording. Vertical vibration of the blade was manually adjusted with a Vibrocheck device (Leica) before slice preparation and set to $0 \mu \mathrm{M}$.

A slice was placed in a modified recording chamber containing ACSF. DCN neurons were identified morphologically through a 40X water immersion objective using DIC-IR optics (Olympus BX50WI, Dulles, VA). Whole-cell patch-clamp recordings were performed using an Axon MultiClamp $700 \mathrm{~B}$ on cells with diameters of $15-20 \mu \mathrm{M}$ in the interpositus and the medial portion of the lateral nucleus. These neurons are regarded as large glutamatergic projection neurons (Aizenman et al., 2003; Huang \& Uusisaari, 2013). Generally, recordings were performed in 2-3 cells from DCN slices per rat after the slices were exposed to ChABC or Vehicle in the medium. Patch pipettes made from borosilicate glass (catalog \#: BF150-86-10; 1.5 mm OD, 0.86 mm ID; Sutter Instrument Company, Novato, CA) were pulled with a P97 Brown-Flaming micropipette puller (Sutter Instrument Company, Novato, CA). The final resistances of pipettes filled with the internal solution [containing (in $\mathrm{mM})$ potassium gluconate $\left(\mathrm{C}_{6} \mathrm{H}_{11} \mathrm{O}_{7} \mathrm{~K}\right)$ 140, $\mathrm{MgCl}_{2} \cdot 6 \mathrm{H}_{2} \mathrm{O}$ 4.6, 
HEPES 10, EGTA 10, $\mathrm{Na}_{2}$ ATP 4.0, pH $7.3(\mathrm{KOH})$ ] were between 5 and $8 \mathrm{M} \Omega$. Data were lowpass filtered at $2 \mathrm{kHz}$ and acquired at $20 \mathrm{kHz}$. Membrane properties were measured when the neuron had stabilized for 5 min after the whole-cell configuration was achieved. Quantitative analysis included resting membrane potential measured directly upon breakthrough in whole-cell configuration, input resistance based on membrane potential changes to depolarizing current injections immediately after whole cell configuration, action potential (AP) threshold, current required for eliciting the first $A P$, half-width of elicited AP (APD50) including rising and falling phases, amplitude of elicited AP, the number of elicited APs, latency to the first AP elicited by a 250-ms duration depolarizing current injection, peak amplitude of the after-hyperpolarization (AHP), interval between first and second evoked action potentials (S1S2 interval), current required for hyperpolarization-induced rebound spikes, and the properties of rebound spikes. Recordings were only accepted if the resistance of initial seal formations were greater than $1 \mathrm{G} \Omega$ and rejected if their output was unstable or series resistance changed by more than $20 \%$. To obtain an accurate measurement of neuronal excitability independent of membrane potential changes, continuous direct current was applied through the recording electrode to hold the cell at a $-70 \mathrm{mV}$ baseline. All recordings were made at room temperature. All electrophysiological data were recorded online using Clampex 10.0 software (Molecular Devices, LLC.). Standard off-line analyses were conducted using Clampfit 10.0 (Molecular Devices, LLC.).

\subsubsection{ChABC Degradation of PNN}

\subsubsection{1. $C h A B C$ in vivo}

A $0.5 \mu \mathrm{L}$ solution containing either $\operatorname{ChABC}(0.01 \mathrm{U} / \mu \mathrm{g} /$ side $/ 0.5 \mu \mathrm{l})$ or vehicle $(0.1 \mathrm{M}$ PBS $)$ was infused into the left AIN. We chose to perform a unilateral infusion because a number of studies have demonstrated that the ipsilateral $\mathrm{DCN}$ is largely responsible for the $\mathrm{CR}$ when testing the ipsilateral eye (Bracha et al., 1997; Campolattaro \& Freeman, 2009; Freeman et al., 1995; Gerwig et al., 2006; Miller et al., 2003). Infusate was injected using a microinfusion pump at a rate 0.5 
$\mu \mathrm{L}$ / minute and the infusion cannula (C317I/SPC, INTERNAL 30GA, Plastics One, Roanoke, VA) was kept in place for another 2 minutes to allow diffusion into the cerebellar tissue. Afterwards, the infusion cannula was removed and replaced with a dummy cannula for the remainder of the experiment. Immediately following removal, sterile water was run through the infusion cannulae to ensure none had clogged. Rats were returned to their home cage for 4 days following ChABC infusion to allow for PNN digestion. The dosage and timing of ChABC infusion and timing of PNN digestion was derived from the literature (Gogolla et al., 2009; Hirono et al., 2018; Xue et al., 2014). Littermates were randomly assigned to the ChABC or vehicle group during surgery and attempts were made to ensure equal numbers of males and females were included in each group.

\subsubsection{ChABC in vitro incubation}

A stock solution of $10 \mathrm{U} / \mathrm{mL}$ of $\mathrm{ChABC}$ was made according to manufacturer specifications. During slice incubation, of ACSF for a final ChABC concentration of $0.25 \mathrm{U} / \mathrm{mL}$ or $250 \mu \mathrm{L}$ of the $0.01 \%$ BSA vehicle solution was added into ACSF as a vehicle. Tissue slices were cut at $350 \mu \mathrm{m}$ and incubated in either $\mathrm{ChABC}$ or vehicle medium. A pilot experiment was done by incubating brain slices with either ChABC or vehicle medium for $4,5,6$, and 8 hours, respectively, in order to determine the optimal ChABC incubation duration for PNN disruption. Our data indicated an incubation time of 8 hours was best-suited for ChABC digestion and this time was used for electrophysiological recordings as well as processing of in vitro tissue.

\subsubsection{Tissue Processing}

In vitro electrophysiology tissue slices were transferred to $4 \%$ paraformaldehyde for 5 days then transferred to $30 \%$ sucrose for cryoprotection until they sank. Following cryoprotection, tissue was re-sectioned on a freezing microtome (HM 450 sliding microtome, Microm of Thermo Fisher

Scientific) at $40 \mu \mathrm{m}$. Tissue was either mounted onto $3 \%$ gelatin slides or placed into cryoprotection in $-20^{\circ} \mathrm{C}$. 
Rats used for the ontogeny analysis, in vivo ChABC degradation, and/ or eyeblink conditioning were given a subcutaneous injection of ketamine hydrochloride $(80 \mathrm{mg} / \mathrm{kg})$ mixed with xylazine $(8.0 \mathrm{mg} / \mathrm{kg})$. Animals were perfused transcardially with $0.9 \%$ saline $(\mathrm{pH} 7.4$ at room temperature) followed by $4 \%$ paraformaldehyde. Brains were collected and placed in fixative until ready for processing. Brains were transferred to $30 \%$ sucrose for cryoprotection until they sank, then 40 $\mu \mathrm{m}$ sections were cut on a freezing microtome. Tissue was either mounted onto $3 \%$ gelatin slides or placed into cryoprotection at $-20^{\circ} \mathrm{C}$.

\subsubsection{PNN immunofluorescence}

Mounted sections from both the in vitro and in vivo experiments were washed in $0.1 \mathrm{M}$ phosphate buffered saline (PBS) - 1\% Tween then blocked for $2 \mathrm{~h}$ with $5 \%$ normal donkey serum or normal goat serum and $3 \%$ BSA before being incubated with primary antibodies overnight at $4^{\circ} \mathrm{C}$. Following washing in 0.1MPBS, sections were incubated in secondary antibody and $1 \%$ blocking solution for $4 \mathrm{~h}$. After completing the secondary antibody reaction, sections were washed again in $0.1 \mathrm{M}$ PBS. Following the final wash, sections were cover-slipped with DAPI Fluoromount-G mounting media and \#1.5 coverslips (Fisher Scientific). Manufacturers' information for primary antibodies, secondary antibodies, and sera can be found in Table 1.

Table 3.1. Materials used in Tissue Processing

\begin{tabular}{|l|}
\hline Item (manufacturer) \\
\hline Chondroitinase ABC (Sigma, cat\#: C3667) \\
\hline Biotin conjugated wisteria floribunda lectin (EY Laboratories Inc., cat\#: BA-3101-1) (1:1000) \\
\hline PSD-95 (Thermofisher Scientific, cat\#: MA1-046) (1:2000) \\
\hline Gephyrin B-4 (Santa Cruz Biotech, cat\#: sc-55469) (1:400) \\
\hline Anti-MAP2, clone AP20 (EMD Millipore, cat\#: MAB3418) (1:200) \\
\hline Donkey Anti-goat A488 (Thermofisher Scientific Inc., Invitrogen, cat\#: A-11055) (1:500) \\
\hline
\end{tabular}


Goat Anti-mouse A546 (Thermofisher Scientific Inc., Invitrogen, cat\#: A-21045) (1:500)

Goat Anti-mouse A488 (Thermofisher Scientific Inc., Invitrogen, cat\#: A-11029) (1:500)

Streptavidin, Alexa Fluor 647 conjugate (Thermofisher Scientific Inc., Invitrogen, cat\#: S-

21374) (1:500)

Normal donkey serum (Millpore Sigma, cał: S30-100KC)

Normal goat serum (Thermofisher Scientific Inc., Gibco, cat\#: PCN5000)

Bovine serum albumin (Millipore Sigma, cat\#: A3294)

DAPI Fluoromount G (SouthernBiotech, cat\#: 0100-20)

\subsubsection{Image Acquisition}

Neuronal and PNN immunofluorescent reactivity in the rat cerebellum was visualized using a confocal laser-scanning microscope (Zeiss LSM 710; Carl Zeiss International). Images were acquired using 405, 488, 546, and $633 \mathrm{~nm}$ lasers, sequential multichannel line scan, and with filters set manually to detect the spectral peak of each fluorophore. Cells were imaged at 20X (NA 0.4) and 63X oil-immersion (NA 1.4) objectives. Raw images were exported to Zen Lite 2009 and Adobe Photoshop CC 2017 to make minor adjustments to the brightness and contrast. Raw images were also exported to FIJI (ImageJ, NIH) to determine the percentage of WFA positive (WFA+) neurons (neurons with a PNN) at multiple time points of development (P12, P16, P18, P30, 3 months, and 6 months) and following either in vitro exposure or in vivo infusion of ChABC or vehicle solution. For PNN analysis, an average of 6 sections per animal were imaged; images were analyzed between the following coordinates (Bregma: 11.52mm, Interaural: -2.52 $\mathrm{mm}$ and Bregma: $10.68 \mathrm{~mm}$, Interaural: $-1.68 \mathrm{~mm}$ ). Each section was imaged bilaterally with the imager blind to the side. In FIJI, the channels were separated in order to compare the number of microtubule-associated protein 2-positive (MAP2+) cells to the WFA+ cells to obtain a normalized count for each image. MAP2 is a protein found in both developing and adult neurons and can be 
used as a biomarker for neurons (Dehmelt \& Halpain, 2004; Kindler \& Garner, 1994; Matus et al., 1990; Tucker et al., 1988). Once channels were separated, a background subtraction was performed to further distinguish WFA+ or MAP2+ cells. Images were thresholded to produce binary images and then particle analysis was used to generate a count of WFA+ or MAP2+ cells. Counts were made every ten slices and the average number of WFA+ cells divided by the average number of MAP2+ cells and then multiplied by 100 to generate a percentage of PNN+ neurons.

\subsubsection{Statistical Analysis}

Data are presented as mean and \pm SEM. One-way ANOVA, two-way ANOVA, and paired and unpaired t-tests were calculated in IBM SPSS Statistics (Ver.26.00.0; IBM Corp.) with $p<0.05$ as the criterion for significance. 


\subsection{Results}

\subsubsection{Ontogenetic Differences in the AIN}

We examined WFA reactivity of MAP2+ cells in the AIN at six time points (P12, P16, P18, P30, 3 months, and 6 months) (Fig 1) and differences were found as a result of age, $[F(5$, $37)=47.75, p<.000]$. Figure 3.1 shows that at P12 and P16 little WFA reactivity was seen but by $\mathrm{P} 18$ and $\mathrm{P} 32$, there was a dense PNN surrounding the cell body as indicated by high WFA reactivity. $\mathrm{P} 12$ and $\mathrm{P} 16$ rats had a lower percentage of $\mathrm{PNN}+$ neurons compared to rats at P18, P30, 3 months, and 6 months. Figure 3.1 also shows higher-resolution images of WFA reactivity at $\mathrm{P} 12, \mathrm{P} 30$, and 3 months. There
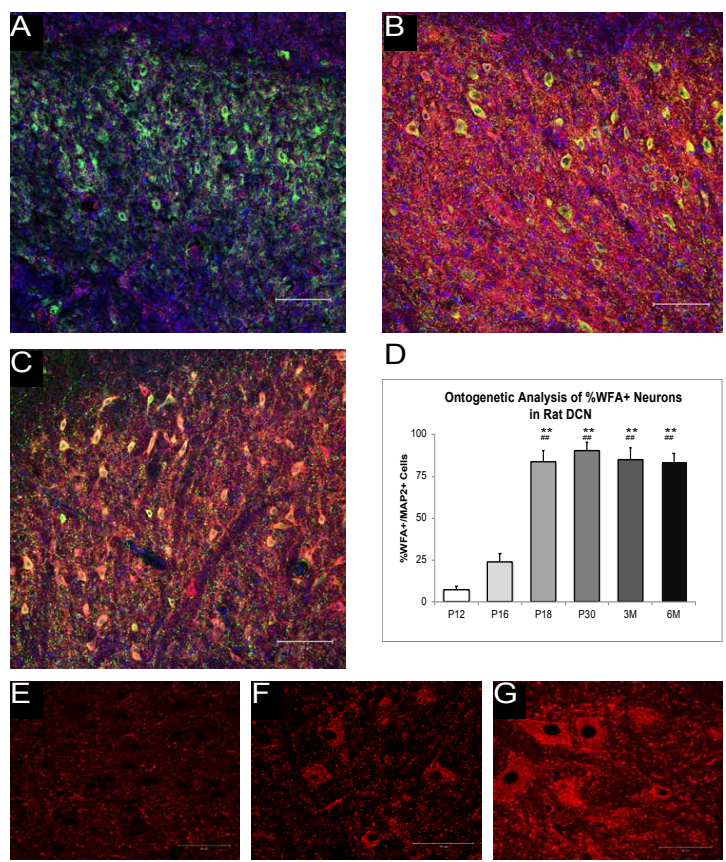

Figure 3.1. Ontogeny of the PNN in the AIN

A-C. shows the WFA reactivity (red), DAPI (blue), and MAP2 reactivity (green) in the rat AIN at P12 (A), P18 $(B)$, and $P 30(C)$ at 20x. D. shows the WFA reactivity at the studied ontogenetic timepoints. There is an increase in the WFA reactivity between P12 and P18 but no significant changes found after this point. E-G show WFA reactivity alone at P12 (E), P30 (F), 3 months $(G)$ at $63 x .{ }^{* *}=p<.01$ at $P 12, \# \#=p<.01$ at P16

were no significant changes in the percentage of PNN+ neurons in the AIN from P18 to 6 months.

\subsubsection{PNN Digestion}

In Vivo alters

\section{Eyeblink}

\section{Conditioning}

\subsubsection{In Vivo PNN}

Digestion

We

examined PNN

function

by

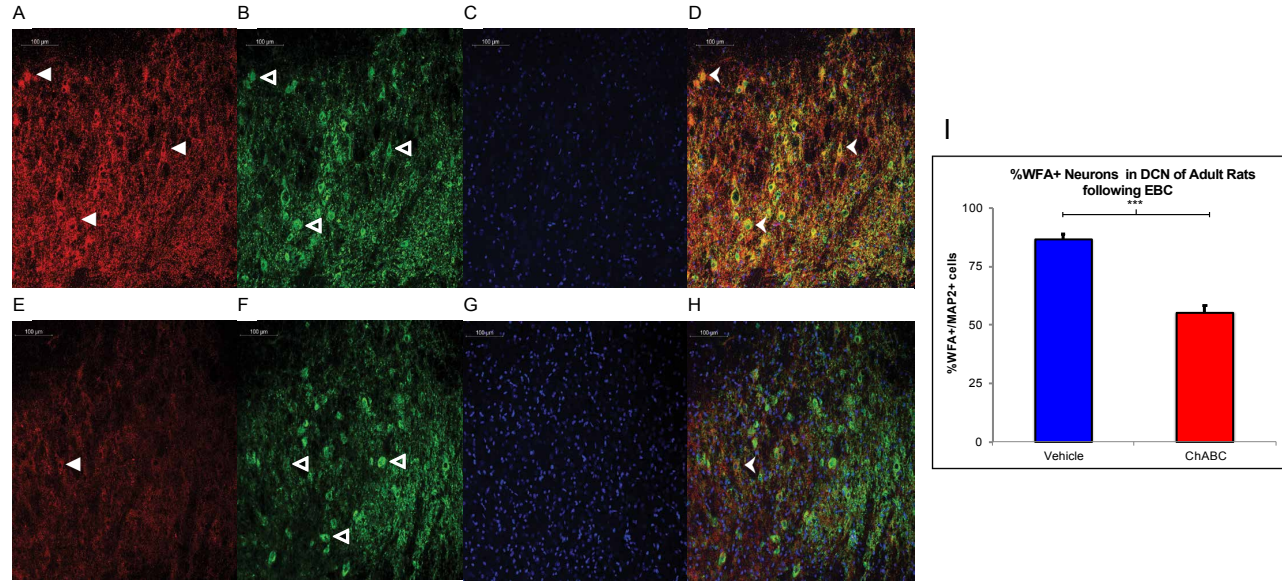

Figure 3.2. In Vivo Digestion of the PNN following EBC

A-D. Rats infused with vehicle show high WFA (red, panel A) reactivity with some PNN noted with white arrowheads as well as MAP2 reactive neurons (green, panel B) with some neurons noted with the open arrowheads and DAPI (blue, panel C) with all four channels merged in D. Three WFA+ neurons are marked with the winged arrowhead in D. E-H. Rats infused with ChABC have less WFA (red, panel E) labeling in comparison and in the merged image, there is only one WFA+ cell. I. A two-tailed unpaired t-test found $C h A B C$ infusion prior to EBC successfully decreased WFA reactivity in the adult rat AIN. ${ }^{* * *}=p<0.001$ 
performing bilateral cannulae implants into the AIN of adult rats with one side being infused with $\mathrm{ChABC}$ and the other with vehicle. The

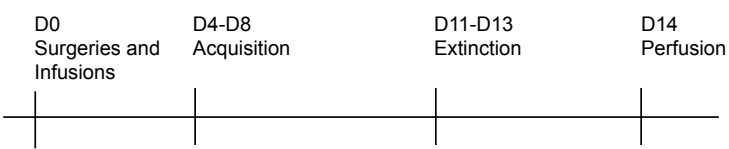

PNN in the AIN on the side that received the enzymatic infusion was found to be digested 4 days post-infusion $(42.38 \pm 5.24 \%)$ compared

Figure 3.3. Behavioral Timeline A simple schematic showing the timeline of the behavioral experiments. Animals had 1 session of tone-shock acquisition for 5 days. 24 hours later they had 1 session of extinction for 3 days. 2 weeks post-surgery the animals were given transcardial perfusion for tissue processing.

to the side receiving vehicle $(68.78 \pm 5.14 \%), p$

$<.000$. Although other brain areas have significant PNN digestion that occurs more quickly following ChABC infusion

(Gogolla et al., 2009; Xue et al., 2014), the DCN requires an extended time to achieve PNN digestion at similar concentrations (Hirono et al., 2018). We found that this level/amount of degradation to A

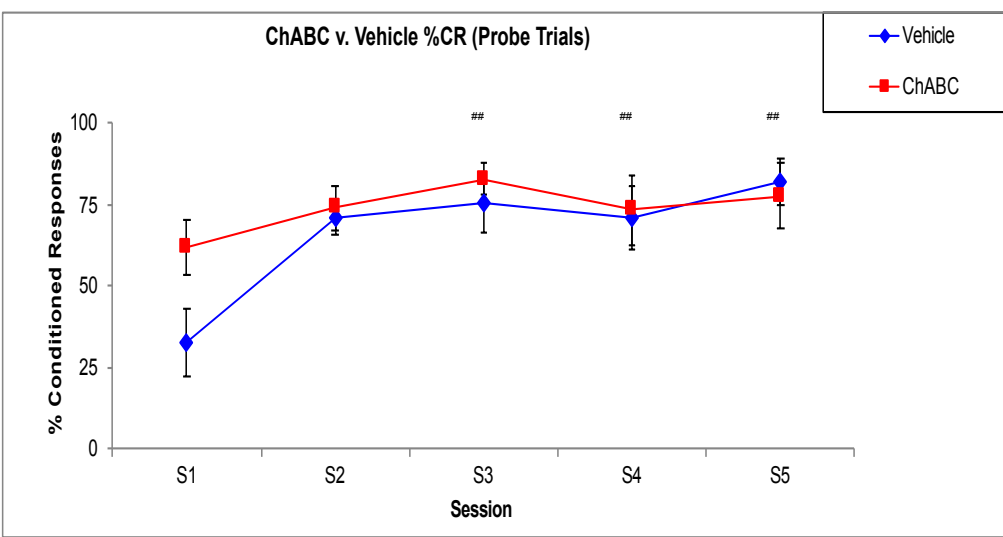

the PNN was present for approximately one month following infusion of ChABC (data not shown). The reassembly of the PNN after a month demonstrated that $\mathrm{ChABC}$ infusion into the $\mathrm{DCN}$ was a temporary digestion of the PNN (Brückner et al., 1998).

B

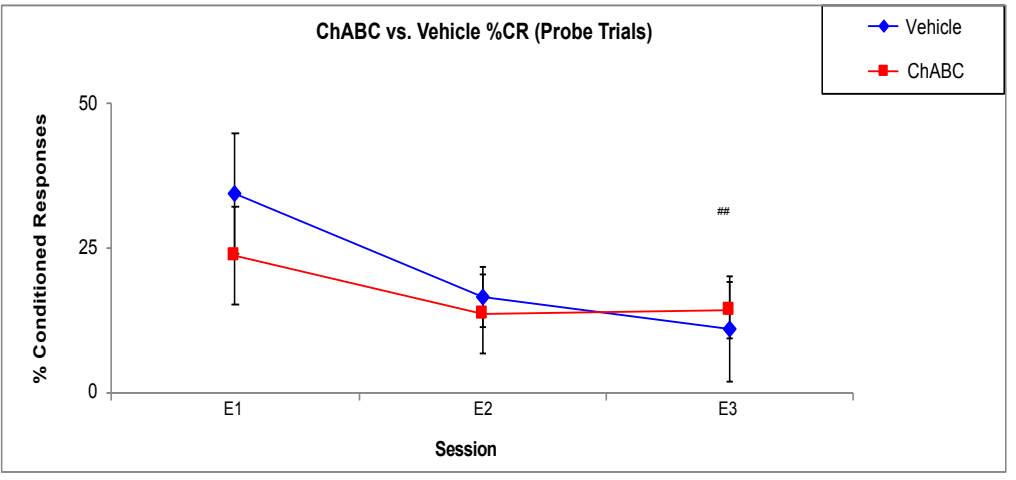

Figure 3.4. Percent Conditioned Responses between ChABC and Vehicle Rats during Acquisition. A. There were no significant differences in \%CRs between the two groups but there were significant sessions differences, showing both groups are capable of learning EBC. B. There were differences between sessions during extinction. \# $=p<.05$ for session 
Rats were removed from the study if their EMG signal-to-noise ratio was so small it was impossible to distinguish their responses from baseline activity. Eighteen adult male and female rats (at least P90) were included in the behavioral data analysis after five sessions of paired EBC ( $\mathrm{n}=9 \mathrm{ChABC}$ $(6 F, 3 M), n=9$ vehicle $(5 F, 4 M)$ on final acquisition session) and up to three sessions of tone-only extinction $(\mathrm{n}=7$ ChABC (4F,3M), $\mathrm{n}=8$ vehicle $(4 F, 4 M)$ on final extinction session) following infusion of

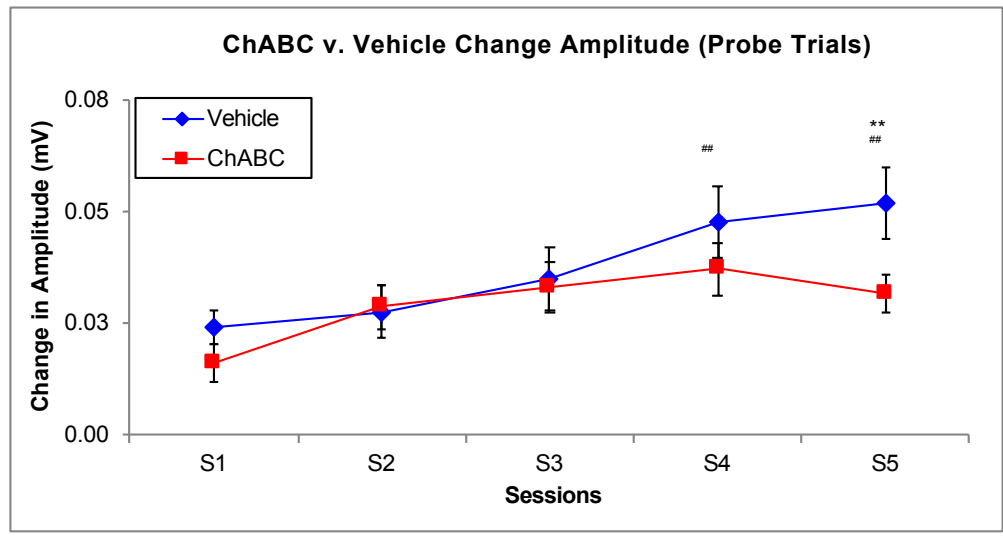

B

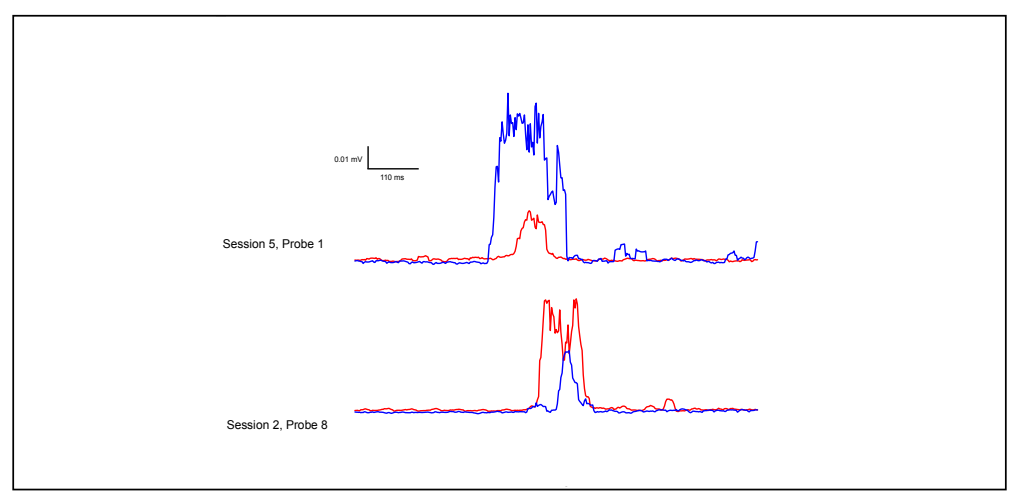

Figure 3.5. Amplitude of the Conditioned Response between ChABC and Vehicle Rats.

A. On S5, the vehicle group had a noticeably higher amplitude on the final acquisition session. There were also significant session differences on S4 and S5 showing the amplitude size significantly increased over the acquisition period. B. the amplitude of a vehicle and ChABC infused rat changed from S3 (bottom traces) to S5 (top trace). \#\# $=p<.05$ for session, ${ }^{* *}=p<.01$

ChABC or PBS vehicle into the AIN. These numbers decreased as the experiment progressed because grooming disrupted the EMG signal over the course of the experiment by displacing the EMG wires in the eyelid. Even if an animal had poor EMG signal and their behavioral results could not be analyzed for that particular day, they were still placed in the chamber and exposed to the stimuli and brain tissue was stained and imaged to confirm ChABC digestion. Figure 3.2 shows ChABC was capable of successfully digesting the PNN; we found that adult rats receiving infusion of ChABC prior to EBC had fewer MAP2+ neurons associated with the PNN $(55.1 \pm 3.2 \%)$ compared to vehicle who had EBC $(86.4 \pm 2.5 \%), p<.000$. 


\subsubsection{EBC Acquisition}

Figure 3.3 shows a timeline for the behavioral experiments. Figure 3.4 shows there was an increase in $\%$ CRs for both groups across sessions, $[F(4,77)=5.82, p<.000]$, with Session 1 (S1) having lower $\%$ CRs than S3 $(p=.001)$, S4 $(p=$ $.016)$, and $\mathrm{S} 5(\mathrm{p}=.001)$ but there were no overall differences in \%CRs between groups.

There were several changes to the parameters of CRs during paired EBC, which are important tools for measuring an animal's ability to
A

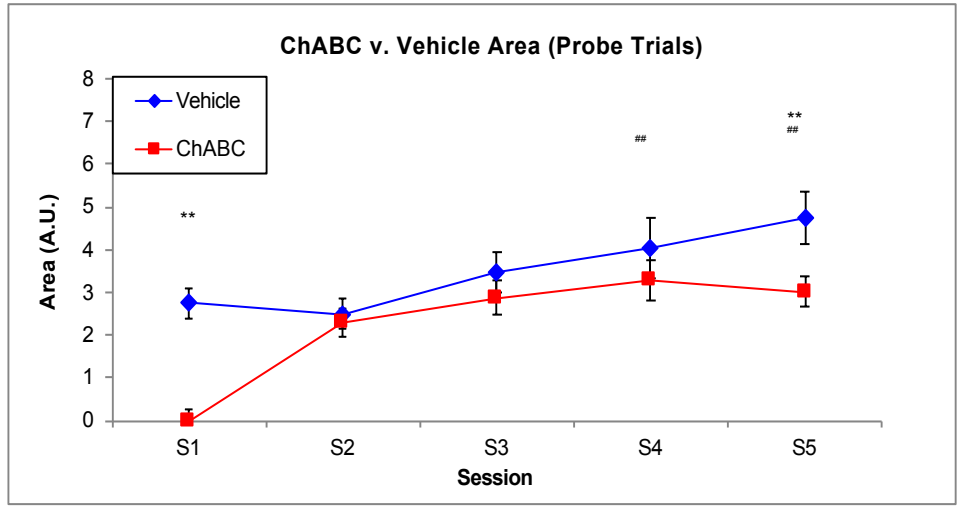

B

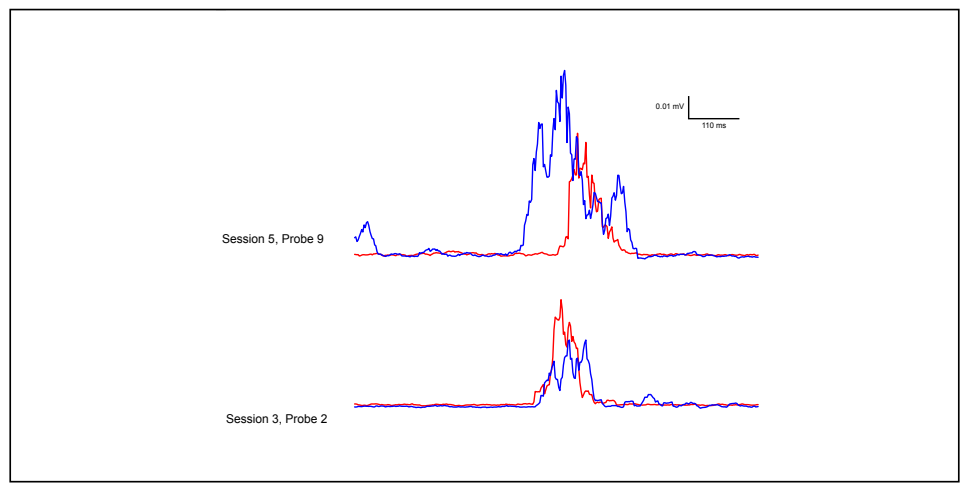

Figure 3.6. Area of the Conditioned Response between ChABC and Vehicle Rats.

A. On both S1 and S5, the vehicle group had a noticeably higher area under the curve. B. The area of a vehicle and ChABC infused rat changed from S3 (bottom traces) to S5 (top trace). \#\# $=p<.05$ for session, ${ }^{* *}=p<.01$

learn EBC. As expected from previous research (Burhans et al., 2013; Schreurs et al., 2013), Figure 3.5 shows session differences in CR amplitude were observed $[F(4,73)=4.68, p=.002]$, with S1 having lower amplitudes than S4 $(p=.008)$ and S5 $(p=.004)$. There was also a session by group interaction for $C R$ amplitude $[F(9,73)=3.43, p=.001]$. Rats that received $C h A B C$ had lower CR amplitudes during the final session (S5) compared to vehicle animals (Fig 5). Representative traces of individual probe trials are shown for a ChABC-infused subject (red) and a Vehicle-infused subject (blue) in the in Fig 5B.

As expected with robust classical conditioning of the EBC, there were session differences in the CR area, seen in Figure 3.6, $[F(4,73)=5.28, p=.001]$ which were smaller on S1 compared to S4 $(p=.008)$ and S5 $(p=.003)$. More importantly, the ChABC group had reduced CR area 
compared to the vehicle group, $[F(1$, 73) $=7.72, p=.007]$. ChABC infused rats had smaller CR area compared to vehicle animals on both the first $(2.74$ $\pm 0.35 \mathrm{AU}$ in vehicle, $0.000 \pm 0.26 \mathrm{AU}$ in $\mathrm{ChABC}, \mathrm{p}=0.02$ ) and final session of acquisition $(4.75 \pm 0.62 \mathrm{AU}$ in vehicle, $3.01 \pm 0.37 \mathrm{AU}$ in $\mathrm{ChABC}, \mathrm{p}$ $=0.03)($ Fig 6). Representative individual traces on probe trials ( $C h A B C$ in red, vehicle in blue) for two sessions of acquisition, corroborate these averaged data (Fig 6B). The changes observed in amplitude and area suggest that digestion of the PNN diminished the size of the conditioned response.

A
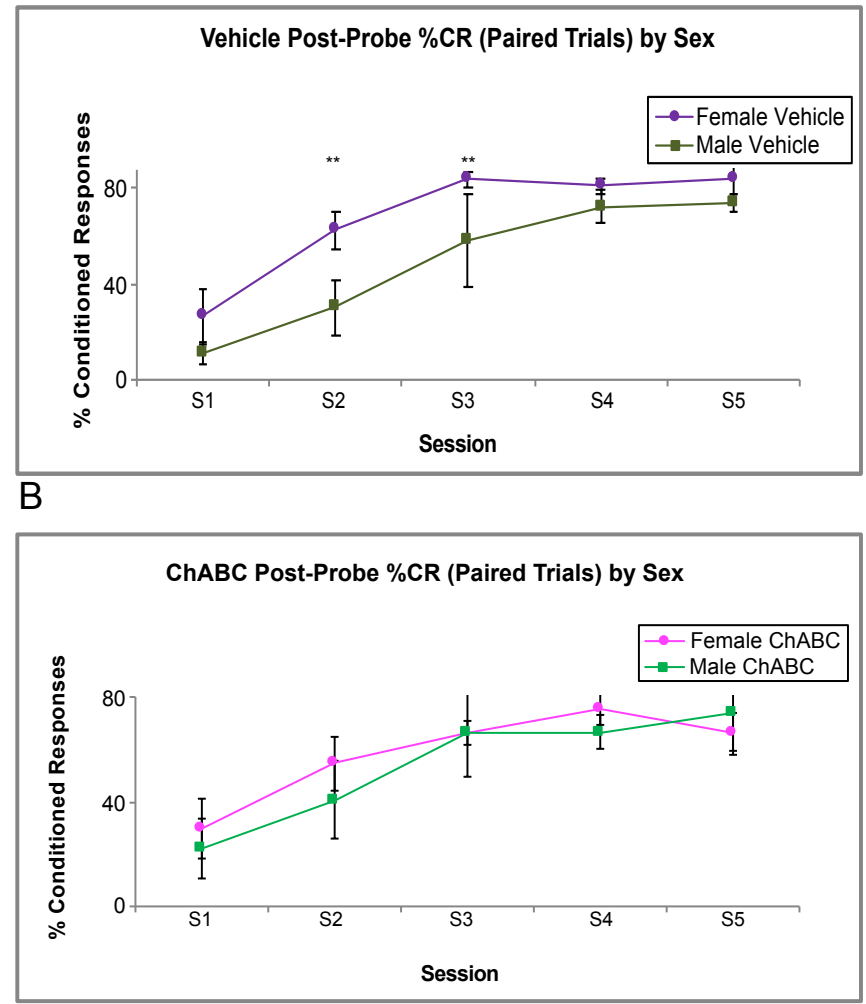

Figure 3.7. \%CR Sex Differences between ChABC and Vehicle Rats during Acquisition. A. shows female rats in the vehicle group have higher $\%$ CRs compared to their male counterparts on the first toneshock trials that follow a probe trial. B. shows that this difference is not present in the rats infused with ChABC. ** $-p<.01$

Following $\mathrm{ChABC}$ or vehicle infusion, there were session differences associated with peak eyeblink latency, $[F(4,74)=8.17, p<.000]$ with the eyeblink peak response occurring earlier on S3 $(p=0.03)$, S4 $(p<.000)$, and S5 $(p<.000)$ compared to S1 (Smith-Bell et al., 2012). The eyeblink response grew faster by the end of acquisition. There were no significant group differences in the peak latency of the $C R(F<1)$.

No significant differences were found in any of the UR measures between the animals infused with $C h A B C$ versus vehicle suggesting that there were no effects of $C h A B C$ on the motor output pathway (F's $<1)$. 


\subsubsection{EBC Extinction}

Figure 3.4 shows the \%CRs during the three extinction sessions. Analysis yielded only session differences, $[F(2,43)=3.36, p=.044]$, with the final extinction session (E3) having lower \%CRs than the first extinction session (E1) (Fig 4B). There were no significant differences in any of the other conditioned response parameters investigated during extinction $(F ' s<1)$.

\subsubsection{Sex Differences}

There were some effects of sex on \%CRs during EBC seen in Figure 3.7. The vehicle group exhibited sex

A
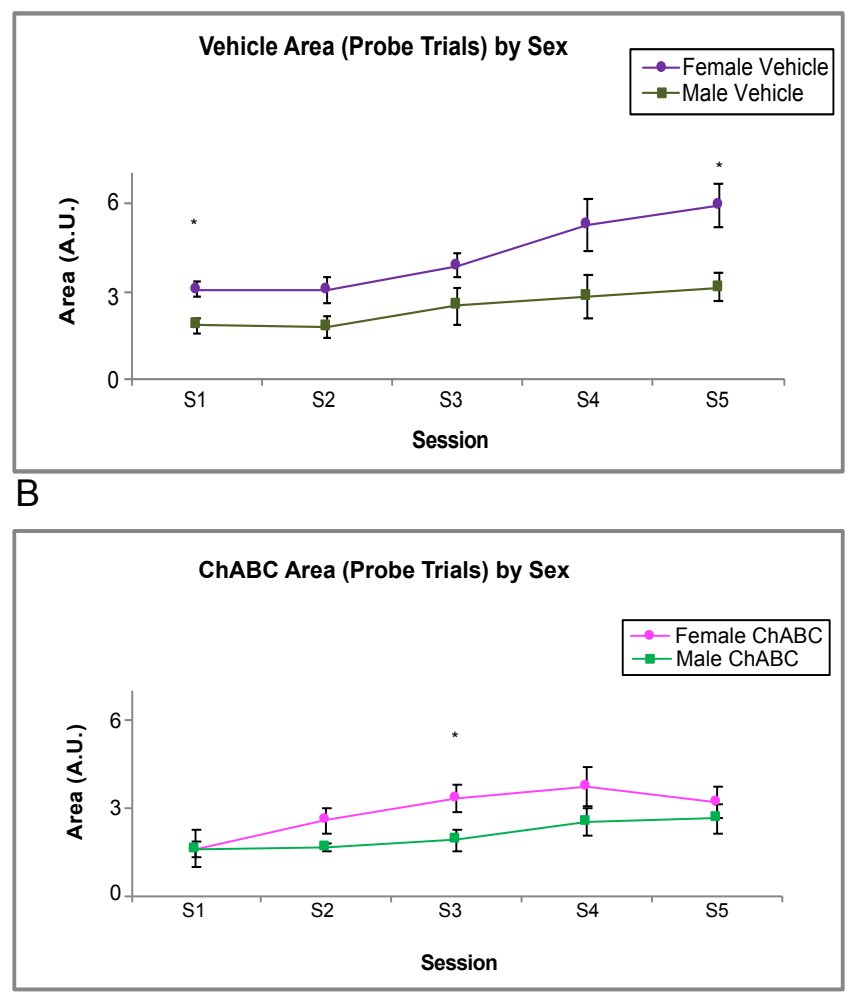

Figure 3.8. Area Sex Differences between ChABC and Vehicle Rats during Acquisition. A. shows female rats in the vehicle group have higher area compared to their male counterparts on probe trials on $\mathrm{S} 1$ and S5. B. shows that female rats infused with ChABC also have a higher area than their male counterparts on S3. * $p<.05$

differences with female rats having marginally higher \%CRs than males during some acquisition sessions, $[F(1,54)=3.91, p=.053](F i g .7 A)$. These sex differences were not evident in the ChABC group $(F<1)$. In the vehicle group, sex differences were found in $\% C R$ s on post-probe trials, the paired trials that immediately followed the tone-alone probes that were presented every ten trials during acquisition, $[F(1,32)=9.66, p=.004]$, with female rats having higher $\% C R s$ on the next reinforced trial that followed the probe trial compared to their male counterparts. Again, the ChABC group did not exhibit these sex differences (Fig 7B). 
Lastly,

Figure 3.8 shows

an effect of sex

on CR area. This

was observed for

both the vehicle

group, $[F(1,48)=$

27.63, $p<.001]$
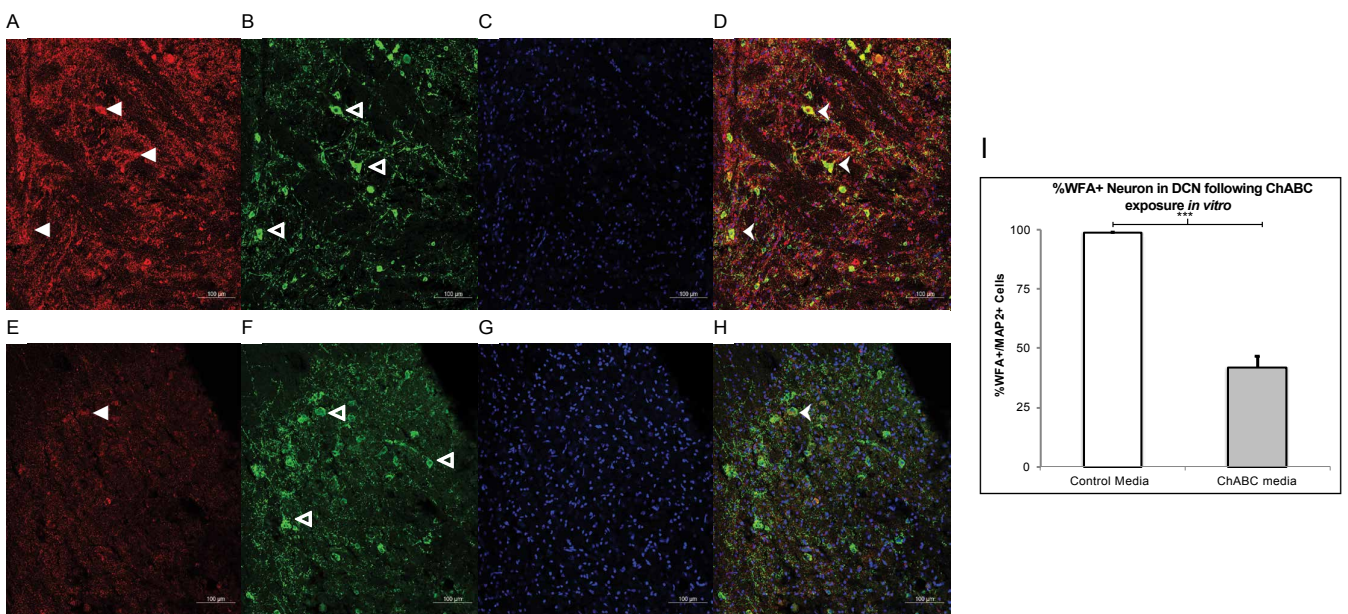

and the ChABC

group, $[F(1,44)=$

7.72, $\mathrm{p}=.008]$

during

Figure 3.9. In Vitro Digestion of the PNN

A-D. Slices exposed with vehicle show high WFA (red, panel A) reactivity as well as MAP2 reactive neurons (green, panel B) and DAPI (blue, panel C) and all channels merged in D. Three WFA+ neurons are marked with the winged arrowhead in D. E-H. Slices exposed with ChABC have less WFA (red, panel E) labeling in comparison and in the merged image, there is only one WFA+ cell. I. A two-tailed paired t-test found ChABC exposure in the electrophysiological bath successfully decreased WFA reactivity in the juvenile rat AIN. ${ }^{* * *}=p<0.001$

acquisition. The female rats in both groups had a larger CR area than the males.

\subsubsection{In Vitro PNN Digestion Alters the Electrophysiological Properties of Neurons in the AIN}

$0.25 \mathrm{U} / \mathrm{mL}$ of ChABC successfully digested the PNN in DCN slices. Figure 3.9 shows AIN tissue that was exposed to ChABC in the medium had lower WFA reactivity compared to tissue placed in the vehicle medium. An unpaired t-test confirmed that the AIN tissue exposed to ChABC had a lower percentage of WFA+ neurons $(41.98 \pm 4.75)$ compared to the vehicle group (98.71 \pm $0.38), p<.000$. Table 2 depicts which membrane properties of neurons in the rat AIN were altered by exposure to ChABC.

Table 3.2: ChABC treatment altered membrane properties of rat $D C N$ neurons 


\begin{tabular}{|c|c|c|c|c|c|c|c|c|c|c|c|}
\hline & $\begin{array}{l}\mathrm{Vm} \\
(\mathrm{mV})\end{array}$ & $\begin{array}{l}\text { Input Res } \\
(\mathrm{M} \Omega)\end{array}$ & $\begin{array}{c}\text { AP } \\
\text { threshold } \\
(\mathrm{mV})\end{array}$ & $\begin{array}{c}\text { Current } \\
\text { required } \\
\text { for evoked } \\
\text { AP (nA) }\end{array}$ & $\begin{array}{l}\text { Latency } \\
\text { (ms) }\end{array}$ & $\begin{array}{l}\text { Amplitude } \\
(\mathrm{mV})\end{array}$ & $\begin{array}{l}\mathrm{APD}_{50} \\
(\mathrm{~ms})\end{array}$ & $\begin{array}{c}\mathrm{APD}_{50} \\
\text { rising } \\
\text { (ms) }\end{array}$ & $\begin{array}{l}\text { APD }_{50} \\
\text { falling } \\
\text { (ms) }\end{array}$ & $\begin{array}{l}\text { AHP } \\
(m V)\end{array}$ & $\begin{array}{c}\text { S1S2 } \\
\text { interval } \\
\text { (ms) }\end{array}$ \\
\hline Vehicle & $-46.93 \pm 1$ & $132.95 \pm 16$ & $-47.90 \pm 2$ & 0.02 & $20.27 \pm 4$ & $69.92 \pm 3$ & 1.04 & 0.40 & 0.64 & $-8.88 \pm 1$ & $17.83 \pm 3$ \\
\hline ChABC & $-46.40 \pm 1$ & $124.03 \pm 1$ & $-42.71 \pm 1^{* *}$ & $0.04^{* *}$ & $45.99 \pm 10^{*}$ & $70.62 \pm 2$ & 0.97 & 0.38 & 0.59 & $-12.46 \pm 1^{*}$ & $31.2 \pm 5^{*}$ \\
\hline
\end{tabular}

Vehicle: Medium treatment; ChABC: ChABC treatment.

Cell number $(\mathrm{n})$ for Vehicle and $\mathrm{ChABC}$ was 16, and 22, respectively. Cells from 5 rats Note that there were group differences in threshold, current required for evoked AP, latency for evoked AP, AHP amplitude, and S1S2 interval. ", " indicate $p<0.05,0.01$, respectively.

AP: Action potential; AP duration was measured at the width of $50 \%$ AP duration (APD 50 ); AHP: Afterhyperpolarization; Input Res: Input resistance.

Figure 3.10 shows neurons exposed to $C h A B C$ required a larger current $(0.04 \pm 0.01 \mathrm{nA})$ to fire an action potential (AP) compared to cells in the vehicle group $(0.02 \pm 0.01 n A), p=0.009$, (Fig 10A) and had a longer latency to evoke an AP (45.99 $\pm 9.51 \mathrm{~ms})$ compared to cells from vehicle animals $(20.27 \pm 4.19 \mathrm{~ms}), \mathrm{p}=0.03$ (Fig 10B). AIN excitatory neurons also showed a prolonged inter-spike interval (S1S2) when exposed to ChABC (31.2 $\pm 5.0 \mathrm{~ms})$ compared to the neurons in the vehicle media $(17.83 \pm 3.14 \mathrm{~ms}), \mathrm{p}=0.04$ (Fig 10C). Figure 3.11 shows ChABCexposed neurons had a larger afterhyperpolarization (AHP) amplitude (-12.46 $\pm 1.12 \mathrm{mV})$ compared to vehicle cells $(-8.88 \pm 1.36 \mathrm{mV}), \mathrm{p}=0.04$ (Fig 11A). The voltage to reach AP threshold was lower following $\mathrm{ChABC}$ exposure $(-42.70 \pm 0.94 \mathrm{mV})$ compared to the vehicle group neurons $(-47.90 \pm 1.84 \mathrm{mV}), \mathrm{p}=.008$ (Fig 11B). Interestingly, there were no differences in the membrane potential $(-46.93 \pm 0.82 \mathrm{mV}$ and $-46.40 \pm 0.69 \mathrm{mV})$ or input resistance $(132.95 \pm 15.6 \mathrm{M} \Omega$ and $124.03 \pm 1.07 \mathrm{M} \Omega$ ) of neurons exposed to ChABC compared to vehicle. These results suggest that digestion of PNN with ChABC in acute AIN slices decreased the intrinsic excitability of large excitatory neurons without affecting other membrane properties.

Table 3.3: ChABC treatment affected the properties of rebound spikes from rat DCN neurons 


\begin{tabular}{lccccccc}
\hline & $\begin{array}{c}\text { Current } \\
\text { required for } \\
\text { evoked RD }(\mathrm{nA})\end{array}$ & $\begin{array}{c}\mathrm{RD} \text { threshold } \\
(\mathrm{mV})\end{array}$ & $\begin{array}{c}\text { RD Amplitude } \\
(\mathrm{mV})\end{array}$ & $\begin{array}{c}\text { RD duration } \\
(\mathrm{ms})\end{array}$ & $\begin{array}{c}\text { RD rising } \\
\text { duration }(\mathrm{ms})\end{array}$ & $\begin{array}{c}\text { RD falling } \\
\text { duration }(\mathrm{ms})\end{array}$ & $\begin{array}{c}\text { AHP } \\
(\mathrm{mV})\end{array}$ \\
\hline Vehicle & $-\mathbf{0 . 2 9}$ & $-50.89 \pm 2$ & $66.49 \pm 2$ & 0.94 & 0.38 & 0.56 & $-12.22 \pm 1$ \\
ChABC & $-\mathbf{0 . 5 9}$ & $-49.03 \pm 2$ & $69.39 \pm 3$ & 1.02 & 0.39 & $33.48 \pm 10$ & 0.60 \\
\hline
\end{tabular}

Vehicle: Medium treatment; ChABC: ChABC treatment.

Cell number (n) for Vehicle and ChABC was 15, and 20, respectively. Cells from 5 rats

Note that there was difference in Current required for evoked rebound spikes $(p=0.0339){ }^{*}$ indicates $p<0.05$

$\mathrm{RD}$ : Rebound spike; RD duration was measured at the width of $50 \% \mathrm{RD}$ duration; AHP: Afterhyperpolarization;

The electrophysiological properties of rebound spikes (RD) are summarized in Table 3.3 and seen in Figure 3.12. Neurons in the $D C N$ slices exposed to $C h A B C$ required a larger current for evoked rebound spikes $(-0.59 \pm 0.11 \mathrm{nA})$ compared to those exposed to the vehicle media ($0.29 \pm 0.01 \mathrm{nA}), \mathrm{p}=0.03$ (Fig 12A). In addition, there were fewer evoked RD in DCN neurons exposed to ChABC $(4.63 \pm 0.9)$ versus those exposed to the vehicle solution $(10.3 \pm 0.74), p=$ 0.0003 (Fig 12B). Increased rebound firing in the DCN is associated with higher likelihood of inducing changes responsible for cerebellar learning (Person \& Raman, 2012; Pugh \& Raman, 2006; Zheng \& Raman, 2010, 2011). Taken together, it is likely that ChABC exposure removed the surrounding PNN resulting in modified the intrinsic membrane properties of the large excitatory neurons within the DCN. 


\subsection{Discussion}

The principal

findings of the present

experiment were: (1)

ChABC was able to

digest PNNs in the rat

$\mathrm{DCN}$ that emerge

almost fully formed at

P18; (2) In vivo

ChABC digestion of

the PNNs in the DCN

had

deleterious

effects on eyeblink

conditioning

particularly on the size

of the conditioned

response without

affecting the

unconditioned response;

properties of large, excitatory neurons in the DCN significantly reducing membrane excitability which has been shown to play a role in eyeblink conditioning.

Our investigation of PNN development in the DCN adds to the current literature that describes the presence of the PNN in this region for adults and at only a few early timepoints. Rats and other animals younger than P18 have difficulty acquiring and retaining EBC and this may be the result of an underdeveloped PNN. Even at P17-P18, rats do not learn EBC with an auditory CS as quickly or as well as they do at P24 (Stanton et al., 1992). This difficulty in training
A

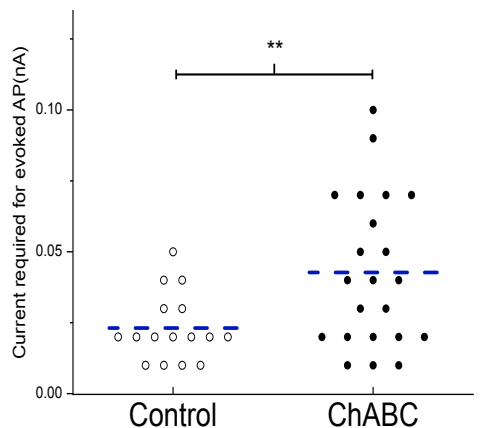

C

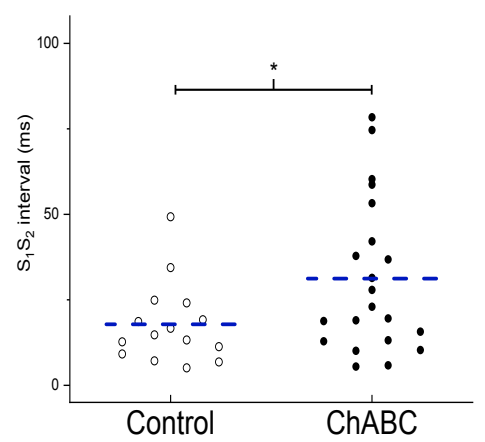

B
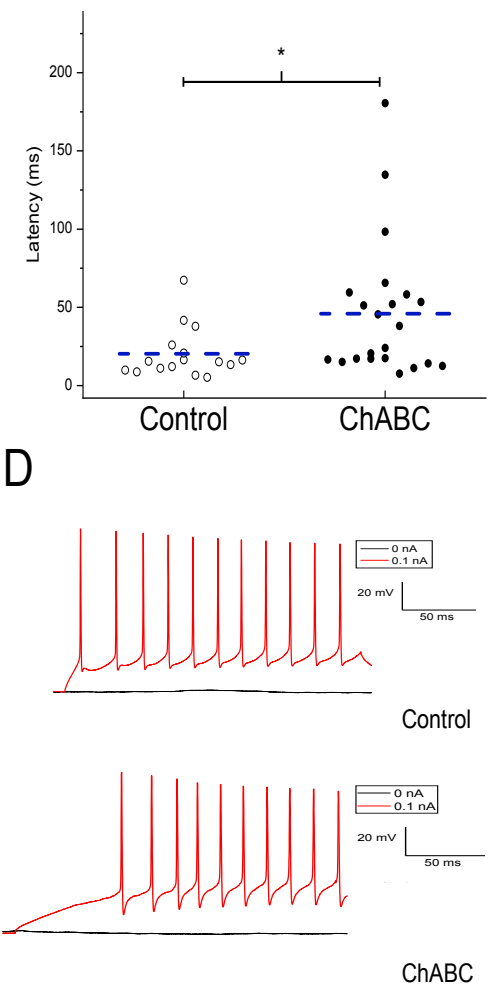

Figure 3.10. ChABC altered the electrophysiological properties of AIN cells. A. shows a higher current is required to evoke an action potential in neurons from ChABC exposed slices. B. shows a significantly longer latency for evoked action potential in neurons from ChABC exposed slices. C. shows a longer S1S2 interval for evoked action potentials in neurons from ChABC exposed slices. D. The typical recordings of action potentials from neurons either in ChABC exposed slices or control medium exposed slices, which were elicited in response to a depolarizing current of $0.1 \mathrm{nA}$. The dashed line indicates the mean. ${ }^{*}=p<.05,{ }^{* *}=p<.01$

(3) Exposure to ChABC in vitro profoundly affected membrane 
rats younger than $\mathrm{P} 17-$

18 with an auditory CS

may also be due in part

to an immature auditory

sensory pathway

(Freeman \& Rabinak, 2005), since training is possible if the CS is a somatosensory stimulus like shock (Schreurs et al., 2013) or vibration

(Goldsberry et al., 2014), because unlike auditory pathways, the somatosensory pathways mature
A

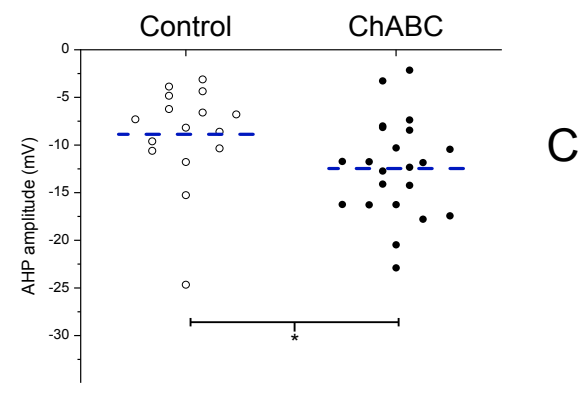

B

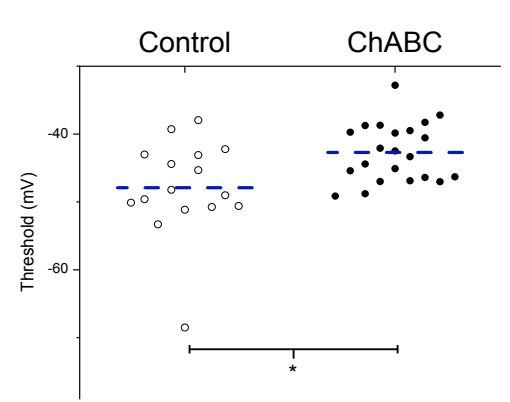

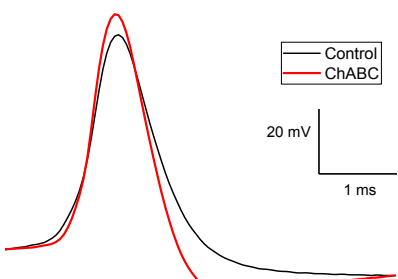

prenatally. Training rats as young as $\mathrm{P} 12$ is possible if the pontine nuclei are directly stimulated as the CS (Freeman \& Rabinak, 2005). The PNN could also be implicated in age-related differences in extinction of EBC. In a study comparing P17 and P24 rats, direct stimulation of the middle cerebellar peduncle as a CS paired with a 25-ms, 3.0-mA (range 2.5-3.5 mA) periorbital stimulation US was used to overcome the immature auditory system so that both ages had strong CRs at the end of acquisition. Even with strong CRs, results showed that P17 animals had a faster rate of extinction compared to P24 animals. P24 animals also had a faster rate of reacquisition following extinction, suggesting that the original memory was better stabilized in P24 rats (Brown \& Freeman, 2014). Although P24 rats are still within their critical period, it is possible that they have more $\mathrm{PNN}+$ cells than preweanlings aged $\mathrm{P} 17$, suggesting they are more likely to stabilize 
the associations learned from EBC. Even rats at A

P24, a "periweanling" age, still diverge in their conditioned behavior compared to adults at P60-P90 (Brown \&

B

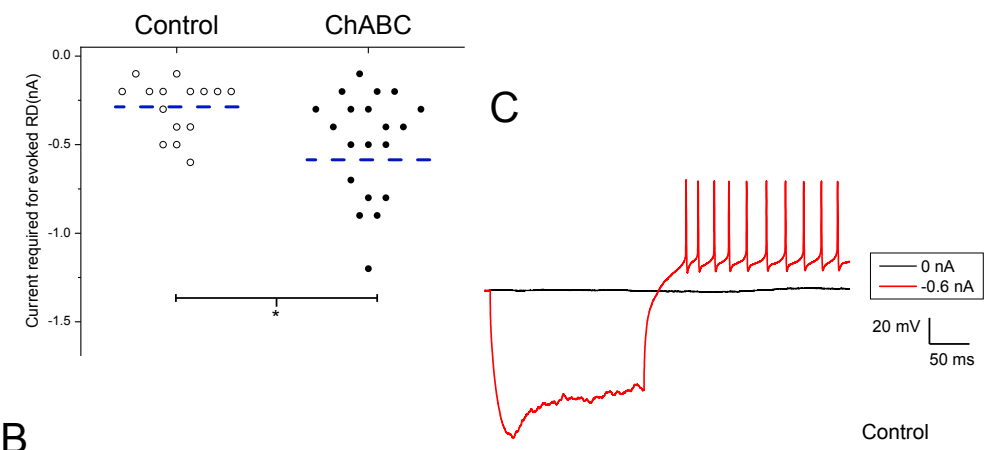

Freeman, 2016). The authors of that 2016 study

found that there were unremarkable differences
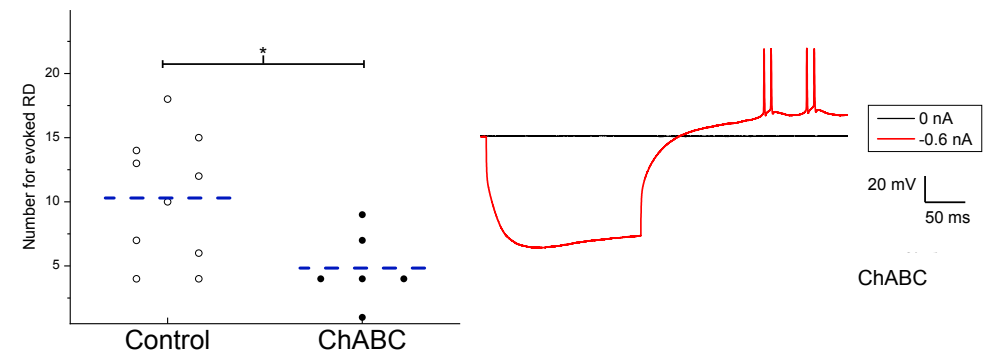

ChABC

between the

periweanlings and adult

rats in acquisition and

extinction following 1,7 ,

or 28 days after

conditioning took place.

Figure 3.12. ChABC exposure changed the properties of hyperpolarization induced rebound spikes in AIN cells. A. shows a higher current is required to evoke a rebound spike in neurons from ChABC exposed slices. B. shows a lower number of evoked rebound spikes in neurons from ChABC exposed slices. Dashed lines indicate the mean. C. The typical recording of rebound spikes from neurons either in ChABC exposed slices or control medium exposed slices, which were elicited in response to a hyperpolarizing current of $-0.6 \mathrm{nA} .{ }^{*}=\mathrm{p}<.05$

However, when they re-exposed periweanlings to the CS-US pairings 29 days following conditioning, they required more training to achieve the high levels of CRs observed during their initial training, suggesting that some memory instability persists in periweanling rats. Together, the behavioral results found by Brown and Freeman as well as our ontogenetic analysis of the PNN and behavior demonstrate that there are age-related differences in an animal's ability to learn EBC. These developmental differences may be dependent on the presence of the PNN in the $\mathrm{DCN}$ as well as other brainstem structures related to EBC. Further examination of the changes to the PNN following EBC at various ontogenetic stages would prove a logical next step. 
ChABC infusion 4 days prior to five sessions of $E B C$ and three sessions of $E B C$ extinction was sufficient to significantly reduce the PNN as well as loose ECM. Exposure to EBC and other stimuli may also remodel the PNN as a result of endogenous enzymatic activity related to learning or changes in the cage environment as observed by others (Carulli et al., 2020; Stamenkovic et al., 2017). Both vehicle and ChABC groups reached our predefined conditioning criterion ( $70 \%$ CR) by S3. We may have missed subtle conditioning differences when comparing between training sessions due to the intensity of our stimuli and the consequent rapid level of learning.

To assess more subtle conditioning differences, we investigated within the acquisition and extinction sessions by assessing \%CRs in 10-trial blocks (Smith-Bell \& Schreurs, 2017). Although all rats acquired equal levels of \%CRs during the first four sessions, group differences emerged during the last session (S5), with ChABC-treated subjects having lower \%CRs than vehicletreated controls. The nature of this difference was explored by examining the final 10 -trial block during one session versus the next session's first 10-trial block (Smith-Bell \& Schreurs, 2017). We found a group difference on the final 10-trial block of $S 4$ and the first 10-trial block of the final session of acquisition (S5). Rats in the vehicle group may have had higher \%CRs compared to those that had been infused with $C h A B C$. This evidence suggests ChABC infused rats were unable to successfully consolidate their memories as well as vehicle rats. This more in-depth analysis within each extinction session revealed that on $\mathrm{E} 1$ there was a group by 10 -trial block interaction. ChABC-infused rats had lower \%CRs, while there were differences between the 10trial blocks, withT1-10 having higher \%CRs than the remaining 10-trial blocks. We also found differences within 10-trial blocks when comparing ChABC and vehicle controls on the second extinction session (E2). Although we followed previously published timelines with ChABC infused degradation in the DCN, additional time may have been needed to achieve the maximum PNN digestion. However, to ensure viable signal quality from the EMG, we had to begin training as rapidly as possible while allowing $\mathrm{ChABC}$ enough time to enzymatically alter the PNN. 
Nevertheless, we did find conditioning parameters that were significantly different between the two groups. Animals with a digested PNN had conditioned eyeblink responses with smaller amplitudes and with smaller areas compared to vehicle rats. Indeed, the amplitude of conditioned eyeblink responses increased in size in vehicle animals from the second session to the final session while ChABC animal amplitude plateaued by the second session. We observed fairly consistent differences in the CR area which can be interpreted as a measure of CR strength. This difference could be an effect of ChABC on the CR output pathway. The smaller amplitude and area of the conditioned eyeblink response in rats with degraded PNN in the AIN may be a result of the decreased intrinsic membrane excitability and altered inhibitory connections onto the DCN. The study by Carulli et al., found reorganization of the inhibitory and excitatory connections of DCN neurons following PNN digestion (Carulli et al., 2020). One reason we did not observe differences in conditioning levels could not only be due to the extent of PNN digestion but also the fact that the DCN neurons remained functional, unlike with the extensive damage observed following cerebellar lesions. Interestingly, other studies have observed impairments of CR amplitude without drastic changes to the CR acquisition following DCN lesions (Perciavalle et al., 2013; Welsh, 1992) while some have used CR amplitude as the measure of cerebellar learning (Kreider \& Mauk, 2010). Since we did not find any differences between the two groups in responding to the US, it is highly unlikely that the observed differences resulted from alterations in the motor performance of ChABC animals. Instead, ChABC-infused rats may not have had well-stabilized associations of the tone-shock pairings with a reduced PNN leading to responses that were decreased in both amplitude and area. Other studies that investigated PNN digestion in the mouse cerebellum and its effect on EBC chose to train mice using air puff as the US (Carulli et al., 2020; Hirono et al., 2018). The lower salience of an air puff US and the lower rate of learning in those studies may have allowed for differences in \%CRs between ChABC and vehicleinfused groups to emerge between sessions on acquisition. The authors did not investigate parameters of the eyeblink response other than percent CR. 
Lastly, we observed significant sex differences during EBC. The females in both the Vehicle and ChABC groups had larger CR areas than their male counterparts during acquisition of EBC. Females in the vehicle group had marginally higher percent CRs during conditioning and significantly higher post-probe percent CRs compared to males in the group suggesting they may have formed stronger associations compared to the males. This has been observed in delay and trace conditioning with female animals having higher \%CRs (Dalla et al., 2009; Dalla \& Shors, 2009; Leuner et al., 2004; Waddell et al., 2010). In comparison, females and males in the ChABC group performed at similar levels. These results confirm that sex is an important biological variable in learning and memory (Bangasser \& Shors, 2007; Chow et al., 2013; Shors \& Miesegaes, 2002) and may indicate the PNN's role (if any) in disorders or diseases that seem to impact one sex more than the other. These data suggest that researchers studying the PNN should consider including animals from both sexes.

The observed changes in vitro, may help explain the observed behavioral changes seen following ChABC infusion to the AIN. We observed that degradation of the PNN in vitro leads to changes in membrane properties of the large excitatory neurons in the DCN. Slices exposed to ChABC had a significantly diminished level of PNN in comparison to slices exposed to the vehicle. The reduction of the PNN decreased the intrinsic membrane excitability of these neurons based on an observed prolonged latency for evoked AP, an extended S1S2 interval, evoked AP needing a larger current, as well as a relatively larger AHP amplitude without altering membrane voltage, input resistance, or amplitude. The ChABC-induced digestion of the PNN altered the membrane properties of DCN neurons modifying the way these cells behaved. In agreement with these results, there are other reports of decreased excitability following PNN removal (Balmer, 2016; Chu et al., 2018). However, others have found increased excitability after ChABC exposure (Dityatev et al., 2007; Hayani et al., 2018). We have observed that eyeblink conditioning is correlated to increased excitability of DCN neurons (Wang et al., 2018; Schreurs, 2019) and others found similar results in the hippocampus (McEchron et al., 2003; Moyer et al., 1996, 2000; 
Oh \& Disterhoft, 2015). We would then expect to observe a decrease in the conditioning of animals exposed to ChABC, since the digestion of the PNN resulted in decreased excitability. These differences may have to do with the location, type of neuron that is being studied, composition of CSPG in PNN, and level of PNN degradation. Although we did not find any significant differences in the PNN in the DCN post $\mathrm{P} 18$, our electrophysiology recordings were made when these animals were still in their periweanling stage, suggesting that there may be differences in the effects of PNN degradation on DCN neurons between animals at this age and adults of 3 months or older. These recordings were made in periweanlings because it is difficult to successfully patch and record neurons in the DCN post P32 (Wang et al., 2018). In the future, we are interested in discerning if the changes we observed following in vitro digestion are also found when breaking down the PNN via ChABC infusion in vivo and performing electrophysiological experiments four days post infusion.

\subsection{Conclusion}

In summary, we found that PNN levels were less prevalent at P12-P16 but increased until P18 and remained stable from this point onward. We observed that digesting the PNN in the rat DCN altered several parameters of EBC and decreased the intrinsic excitability of the PNN+ neurons. These results add to the growing body of work studying the PNN and its function in the CNS. 
Deidre O’Dell: Experimental design, Investigation, Writing - Original draft preparation, Bernard G. Schreurs: Experimental design, Supervision, Writing - Reviewing and Editing, Carrie SmithBell: Validation of behavior data, Writing - Reviewing and Editing, Desheng Wang:

Investigation, Writing - Reviewing and Editing 
Acknowledgements:

National Institute of General Medical Sciences grant T32 GM081741 and Eunice Kennedy Shriver National Institute of Child \& Human development grant HD099338 for funding support. The content is solely the responsibility of the authors and does not necessarily represent the official views of the National Institutes of Health. 


\section{References:}

Aizenman, C. D., Huang, E. J., \& Linden, D. J. (2003). Morphological correlates of intrinsic electrical excitability in neurons of the deep cerebellar nuclei. Journal of Neurophysiology, 89(4), 1738-1747. https://doi.org/10.1152/jn.01043.2002

Alberini, C. M., \& Travaglia, A. (2017). Infantile Amnesia: A Critical Period of Learning to Learn and Remember. Journal of Neuroscience, 37(24), 5783-5795. https://doi.org/10.1523/JNEUROSCI.0324-17.2017

Araque, A., Parpura, V., Sanzgiri, R. P., \& Haydon, P. G. (1999). Tripartite synapses: Glia, the unacknowledged partner. Trends in Neurosciences, 22(5), 208-215. https://doi.org/10.1016/S0166-2236(98)01349-6

Ayers, E. D., White, J., \& Powell, D. A. (2003). Pavlovian Eyeblink Conditioning in Combat Veterans With and Without Post-Traumatic Stress Disorder. Integrative Physiological \& Behavioral Science, 38(3), 230-247.

Baker, K. D., Gray, A. R., \& Richardson, R. (2017). The development of perineuronal nets around parvalbumin gabaergic neurons in the medial prefrontal cortex and basolateral amygdala of rats. Behavioral Neuroscience, 131(4), 289-303. https://doi.org/10.1037/bne0000203

Balmer, T. S. (2016). Perineuronal Nets Enhance the Excitability of Fast-Spiking Neurons. ENeuro, 3(4), ENEURO.0112-16.2016. https://doi.org/10.1523/ENEURO.0112-16.2016

Bangasser, D. A., \& Shors, T. J. (2007). The hippocampus is necessary for enhancements and impairments of learning following stress. Nature Neuroscience, 10(11), 1401-1403. https://doi.org/10.1038/nn1973

Bekku, Y., Saito, M., Moser, M., Fuchigami, M., Maehara, A., Nakayama, M., Kusachi, S., Ninomiya, Y., \& Oohashi, T. (2012). Bral2 is indispensable for the proper localization of brevican and the structural integrity of the perineuronal net in the brainstem and cerebellum. The Journal of Comparative Neurology, 520(8), 1721-1736. https://doi.org/10.1002/cne.23009

Bracha, V., Zhao, L., Wunderlich, D. A., Morrissy, S. J., \& Bloedel, J. R. (1997). Patients with cerebellar lesions cannot acquire but are able to retain conditioned eyeblink reflexes. Brain, 120(8), 1401-1413. https://doi.org/10.1093/brain/120.8.1401

Bradbury, E. J., Moon, L. D. F., Popat, R. J., King, V. R., Bennett, G. S., Patel, P. N., Fawcett, J. W., \& McMahon, S. B. (2002). Chondroitinase ABC promotes functional recovery after spinal cord injury. Nature, 416(6881), 636-640. https://doi.org/10.1038/416636a

Bradshaw, K. P., Velez, D. X. F., Habeeb, M., \& Gandhi, S. P. (2018). Precocious deposition of perineuronal nets on Parvalbumin inhibitory neurons transplanted into adult visual cortex. Scientific Reports, 8(1), 7480. https://doi.org/10.1038/s41598-018-25735-8

Brown, K. L., \& Freeman, J. H. (2014). Extinction, reacquisition, and rapid forgetting of eyeblink conditioning in developing rats. Learning \& Memory (Cold Spring Harbor, N.Y.), 21(12), 696-708. https://doi.org/10.1101//m.036103.114

Brown, K. L., \& Freeman, J. H. (2016). Retention of eyeblink conditioning in periweanling and adult rats. Developmental Psychobiology, 58(8), 1055-1065. https://doi.org/10.1002/dev.21439

Brown, K. L., \& Woodruff-Pak, D. S. (2011). Eyeblink Conditioning in Animal Models and Humans. In J. Raber (Ed.), Animal Models of Behavioral Analysis (Vol. 50, pp. 1-27). Humana Press Inc.

Brückner, G., Brauer, K., Härtig, W., Wolff, J. R., Rickmann, M. J., Derouiche, A., Delpech, B., Girard, N., Oertel, W. H., \& Reichenbach, A. (1993). Perineuronal nets provide a polyanionic, glia-associated form of microenvironment around certain neurons in many parts of the rat brain. Glia, 8(3), 183-200. https://doi.org/10.1002/glia.440080306 
Brückner, G., Bringmann, A., Härtig, W., Köppe, G., Delpech, B., \& Brauer, K. (1998). Acute and long-lasting changes in extracellular-matrix chondroitin-sulphate proteoglycans induced by injection of chondroitinase $\mathrm{ABC}$ in the adult rat brain. Experimental Brain Research, 121(3), 300-310. https://doi.org/10.1007/s002210050463

Brückner, G., Bringmann, A., Köppe, G., Härtig, W., \& Brauer, K. (1996). In vivo and in vitro labelling of perineuronal nets in rat brain. Brain Research, 720(1), 84-92. https://doi.org/10.1016/0006-8993(96)00152-7

Brückner, G., Szeöke, S., Pavlica, S., Grosche, J., \& Kacza, J. (2006). Axon initial segment ensheathed by extracellular matrix in perineuronal nets. Neuroscience, 138(2), 365-375. https://doi.org/10.1016/j.neuroscience.2005.11.068

Burhans, L. B., Smith-Bell, C. A., \& Schreurs, B. G. (2013). Subacute fluoxetine enhances conditioned responding and conditioning-specific reflex modification of the rabbit nictitating membrane response: Implications for drug treatment with selective serotonin reuptake inhibitors. Behavioural Pharmacology, 24(1), 55-64. https://doi.org/10.1097/FBP.0b013e32835d528e

Cabungcal, J.-H., Steullet, P., Morishita, H., Kraftsik, R., Cuenod, M., Hensch, T. K., \& Do, K. Q. (2013). Perineuronal nets protect fast-spiking interneurons against oxidative stress. Proceedings of the National Academy of Sciences of the United States of America, 110(22), 9130-9135. https://doi.org/10.1073/pnas.1300454110

Campolattaro, M. M., \& Freeman, J. H. (2009). An Examination of Bilateral Eyeblink Conditioning in Rats. Behavioral Neuroscience, 123(6), 1346-1352. https://doi.org/10.1037/a0017314

Carbo-Gas, M., Moreno-Rius, J., Guarque-Chabrera, J., Vazquez-Sanroman, D., Gil-Miravet, I., Carulli, D., Hoebeek, F., De Zeeuw, C., Sanchis-Segura, C., \& Miquel, M. (2017). Cerebellar perineuronal nets in cocaine-induced pavlovian memory: Site matters. Neuropharmacology, 125(Supplement C), 166-180. https://doi.org/10.1016/j.neuropharm.2017.07.009

Carstens, K. E., Phillips, M. L., Pozzo-Miller, L., Weinberg, R. J., \& Dudek, S. M. (2016). Perineuronal Nets Suppress Plasticity of Excitatory Synapses on CA2 Pyramidal Neurons. Journal of Neuroscience, 36(23), 6312-6320. https://doi.org/10.1523/JNEUROSCI.0245-16.2016

Carulli, D., Broersen, R., de Winter, F., Muir, E. M., Mešković, M., de Waal, M., de Vries, S., Boele, H.-J., Canto, C. B., De Zeeuw, C. I., \& Verhaagen, J. (2020). Cerebellar plasticity and associative memories are controlled by perineuronal nets. Proceedings of the National Academy of Sciences of the United States of America. https://doi.org/10.1073/pnas.1916163117

Carulli, D., Foscarin, S., Faralli, A., Pajaj, E., \& Rossi, F. (2013). Modulation of semaphorin3A in perineuronal nets during structural plasticity in the adult cerebellum. Molecular and Cellular Neurosciences, 57, 10-22. https://doi.org/10.1016/j.mcn.2013.08.003

Carulli, D., Pizzorusso, T., Kwok, J. C. F., Putignano, E., Poli, A., Forostyak, S., Andrews, M. R., Deepa, S. S., Glant, T. T., \& Fawcett, J. W. (2010). Animals lacking link protein have attenuated perineuronal nets and persistent plasticity. Brain, 133(8), 2331-2347. https://doi.org/10.1093/brain/awq145

Carulli, D., Rhodes, K. E., Brown, D. J., Bonnert, T. P., Pollack, S. J., Oliver, K., Strata, P., \& Fawcett, J. W. (2006). Composition of perineuronal nets in the adult rat cerebellum and the cellular origin of their components. The Journal of Comparative Neurology, 494(4), 559-577. https://doi.org/10.1002/cne.20822

Carulli, D., Rhodes, K. E., \& Fawcett, J. W. (2007). Upregulation of aggrecan, link protein 1, and hyaluronan synthases during formation of perineuronal nets in the rat cerebellum. The Journal of Comparative Neurology, 501(1), 83-94. https://doi.org/10.1002/cne.21231 
Celio, M. R., Spreafico, R., Biasi, S. D., \& Vitellaro-Zuccarello, L. (1998). Perineuronal nets: Past and present. Trends in Neurosciences, 21(12), 510-515. https://doi.org/10.1016/S0166-2236(98)01298-3

Chow, C., Epp, J. R., Lieblich, S. E., Barha, C. K., \& Galea, L. A. M. (2013). Sex differences in neurogenesis and activation of new neurons in response to spatial learning and memory. Psychoneuroendocrinology, 38(8), 1236-1250. https://doi.org/10.1016/j.psyneuen.2012.11.007

Chu, P., Abraham, R., Budhu, K., Khan, U., De Marco Garcia, N., \& Brumberg, J. C. (2018). The Impact of Perineuronal Net Digestion using Chondroitinase $A B C$ on the Intrinsic Physiology of Cortical Neurons. Neuroscience. https://doi.org/10.1016/j.neuroscience.2018.07.004

Cicanic, M., Edamatsu, M., Bekku, Y., Vorisek, I., Oohashi, T., \& Vargova, L. (2017). A deficiency of the link protein Bral2 affects the size of the extracellular space in the thalamus of aged mice. Journal of Neuroscience Research. https://doi.org/10.1002/jnr.24136

Cope, E. C., \& Gould, E. (2019). Adult Neurogenesis, Glia, and the Extracellular Matrix. Cell Stem Cell, 24(5), 690-705. https://doi.org/10.1016/j.stem.2019.03.023

Corvetti, L., \& Rossi, F. (2005). Degradation of chondroitin sulfate proteoglycans induces sprouting of intact purkinje axons in the cerebellum of the adult rat. The Journal of Neuroscience: The Official Journal of the Society for Neuroscience, 25(31), 7150-7158. https://doi.org/10.1523/JNEUROSCI.0683-05.2005

Dalla, C., Papachristos, E. B., Whetstone, A. S., \& Shors, T. J. (2009). Female rats learn trace memories better than male rats and consequently retain a greater proportion of new neurons in their hippocampi. Proceedings of the National Academy of Sciences of the United States of America, 106(8), 2927-2932. https://doi.org/10.1073/pnas.0809650106

Dalla, C., \& Shors, T. J. (2009). Sex differences in learning processes of classical and operant conditioning. Physiology \& Behavior, 97(2), 229-238. https://doi.org/10.1016/j.physbeh.2009.02.035

De Luca, C., \& Papa, M. (2016). Looking Inside the Matrix: Perineuronal Nets in Plasticity, Maladaptive Plasticity and Neurological Disorders. Neurochemical Research, 41(7), 1507-1515. https://doi.org/10.1007/s11064-016-1876-2

de Winter, F., Kwok, J. C. F., Fawcett, J. W., Vo, T. T., Carulli, D., \& Verhaagen, J. (2016). The Chemorepulsive Protein Semaphorin $3 \mathrm{~A}$ and Perineuronal Net-Mediated Plasticity. Neural Plasticity, 2016, e3679545. https://doi.org/10.1155/2016/3679545

Dehmelt, L., \& Halpain, S. (2004). The MAP2/Tau family of microtubule-associated proteins. Genome Biology, 6(1), 204. https://doi.org/10.1186/gb-2004-6-1-204

Dityatev, A., Brückner, G., Dityateva, G., Grosche, J., Kleene, R., \& Schachner, M. (2007). Activity-dependent formation and functions of chondroitin sulfate-rich extracellular matrix of perineuronal nets. Developmental Neurobiology, 67(5), 570-588. https://doi.org/10.1002/dneu.20361

Edamatsu, M., Miyano, R., Fujikawa, A., Fujii, F., Hori, T., Sakaba, T., \& Oohashi, T. (2018). Hapln4/Bral2 is a selective regulator for formation and transmission of GABAergic synapses between Purkinje and deep cerebellar nuclei neurons. Journal of Neurochemistry, 147(6), 748-763. https://doi.org/10.1111/jnc.14571

Fawcett, J. W., Oohashi, T., \& Pizzorusso, T. (2019). The roles of perineuronal nets and the perinodal extracellular matrix in neuronal function. Nature Reviews Neuroscience, 20(8), 451-465. https://doi.org/10.1038/s41583-019-0196-3

Ferrer-Ferrer, M., \& Dityatev, A. (2018). Shaping Synapses by the Neural Extracellular Matrix. Frontiers in Neuroanatomy, 12. https://doi.org/10.3389/fnana.2018.00040

Freeman, J. H., Carter, C. S., \& Stanton, M. E. (1995). Early cerebellar lesions impair eyeblink conditioning in developing rats: Differential effects of unilateral lesions on Postnatal Day 
10 or 20. Behavioral Neuroscience, 109(5), 893-902. https://doi.org/10.1037/07357044.109.5.893

Freeman, J. H., \& Rabinak, C. A. (2005). Eyeblink conditioning in rats using pontine stimulation as a conditioned stimulus. Integrative Physiological and Behavioral Science, 39(3), 180 191. https://doi.org/10.1007/BF02734438

Galtrey, C. M., Kwok, J. C. F., Carulli, D., Rhodes, K. E., \& Fawcett, J. W. (2008). Distribution and synthesis of extracellular matrix proteoglycans, hyaluronan, link proteins and tenascin-R in the rat spinal cord. European Journal of Neuroscience, 27(6), 1373-1390. https://doi.org/10.1111/j.1460-9568.2008.06108.x

Gerwig, M., Hajjar, K., Frings, M., Dimitrova, A., Thilmann, A. F., Kolb, F. P., Forsting, M., \& Timmann, D. (2006). Extinction of conditioned eyeblink responses in patients with cerebellar disorders. Neuroscience Letters, 406(1), 87-91. https://doi.org/10.1016/j.neulet.2006.07.017

Giamanco, K. A., Morawski, M., \& Matthews, R. T. (2010). Perineuronal net formation and structure in aggrecan knockout mice. Neuroscience, 170(4), 1314-1327. https://doi.org/10.1016/j.neuroscience.2010.08.032

Glickstein, M., Strata, P., \& Voogd, J. (2009). Cerebellum: History. Neuroscience, 162(3), 549559. https://doi.org/10.1016/j.neuroscience.2009.02.054

Gogolla, N., Caroni, P., Lüthi, A., \& Herry, C. (2009). Perineuronal nets protect fear memories from erasure. Science (New York, N.Y.), 325(5945), 1258-1261. https://doi.org/10.1126/science.1174146

Goldsberry, M. E., Elkin, M. E., \& Freeman, J. H. (2014). Sensory system development influences the ontogeny of eyeblink conditioning. Developmental Psychobiology, 56(6), 1244-1251. https://doi.org/10.1002/dev.21204

Guirado, R., Perez-Rando, M., Sanchez-Matarredona, D., Castrén, E., \& Nacher, J. (2014). Chronic fluoxetine treatment alters the structure, connectivity and plasticity of cortical interneurons. The International Journal of Neuropsychopharmacology; Oxford, 17(10), 1635-1646. http://dx.doi.org/10.1017/S1461145714000406

Hayani, H., Song, I., \& Dityatev, A. (2018). Increased Excitability and Reduced Excitatory Synaptic Input Into Fast-Spiking CA2 Interneurons After Enzymatic Attenuation of Extracellular Matrix. Frontiers in Cellular Neuroscience, 12. https://doi.org/10.3389/fncel.2018.00149

Heiney, S. A., Wohl, M. P., Chettih, S. N., Ruffolo, L. I., \& Medina, J. F. (2014). CerebellarDependent Expression of Motor Learning during Eyeblink Conditioning in Head-Fixed Mice. Journal of Neuroscience, 34(45), 14845-14853. https://doi.org/10.1523/JNEUROSCI.2820-14.2014

Hensch, T. K. (2005). Critical period plasticity in local cortical circuits. Nature Reviews Neuroscience, 6(11), 877-888. https://doi.org/10.1038/nrn1787

Hettiaratchi, M. H., O’Meara, M. J., Teal, C. J., Payne, S. L., Pickering, A. J., \& Shoichet, M. S. (2019). Local delivery of stabilized chondroitinase ABC degrades chondroitin sulfate proteoglycans in stroke-injured rat brains. Journal of Controlled Release, 297, 14-25. https://doi.org/10.1016/j.jconrel.2019.01.033

Hilbig, H., Bidmon, H. J., Blohm, U., \& Zilles, K. (2001). Wisteria floribunda agglutinin labeling patterns in the human cortex: A tool for revealing areal borders and subdivisions in parallel with immunocytochemistry. Anatomy and Embryology, 203(1), 45-52.

Hirono, M., Watanabe, S., Karube, F., Fujiyama, F., Kawahara, S., Nagao, S., Yanagawa, Y., \& Misonou, H. (2018). Perineuronal nets in the deep cerebellar nuclei regulate GABAergic transmission and delay eyeblink conditioning. Journal of Neuroscience, 3238-17. https://doi.org/10.1523/JNEUROSCI.3238-17.2018

Hou, X., Yoshioka, N., Tsukano, H., Sakai, A., Miyata, S., Watanabe, Y., Yanagawa, Y., Sakimura, K., Takeuchi, K., Kitagawa, H., Hensch, T. K., Shibuki, K., Igarashi, M., \& 
Sugiyama, S. (2017). Chondroitin Sulfate Is Required for Onset and Offset of Critical Period Plasticity in Visual Cortex. Scientific Reports, 7(1), 12646.

https://doi.org/10.1038/s41598-017-04007-x

Huang, S., \& Uusisaari, M. Y. (2013). Physiological temperature during brain slicing enhances the quality of acute slice preparations. Frontiers in Cellular Neuroscience, 7, 48. https://doi.org/10.3389/fncel.2013.00048

Iwata, M., Wight, T. N., \& Carlson, S. S. (1993). A brain extracellular matrix proteoglycan forms aggregates with hyaluronan. Journal of Biological Chemistry, 268(20), 15061-15069.

Kecskes, S., Gaal, B., Racz, E., Birinyi, A., Hunyadi, A., \& Matesz, C. (2015). Extracellular Matrix Molecules Exhibit Unique Expression Pattern in the Climbing Fiber-Generating Precerebellar Nucleus, the Inferior Olive. Neuroscience, 284, 412-421. https://doi.org/10.1016/j.neuroscience.2014.09.080

Kindler, S., \& Garner, C. C. (1994). Four repeat MAP2 isoforms in human and rat brain. Brain Research. Molecular Brain Research, 26(1-2), 218-224. https://doi.org/10.1016/0169328x(94)90093-0

Koekkoek, S. K. E., Den Ouden, W. L., Perry, G., Highstein, S. M., \& De Zeeuw, C. I. (2002). Monitoring Kinetic and Frequency-Domain Properties of Eyelid Responses in Mice With Magnetic Distance Measurement Technique. Journal of Neurophysiology, 88(4), 21242133. https://doi.org/10.1152/jn.2002.88.4.2124

Kreider, J. C., \& Mauk, M. D. (2010). Eyelid Conditioning to a Target Amplitude: Adding How Much to Whether and When. Journal of Neuroscience, 30(42), 14145-14152. https://doi.org/10.1523/JNEUROSCI.3473-10.2010

Krishnaswamy, V. R., Benbenishty, A., Blinder, P., \& Sagi, I. (2019). Demystifying the extracellular matrix and its proteolytic remodeling in the brain: Structural and functional insights. Cellular and Molecular Life Sciences. https://doi.org/10.1007/s00018-01903182-6

Leuner, B., Mendolia-Loffredo, S., \& Shors, T. J. (2004). High levels of estrogen enhance associative memory formation in ovariectomized females. Psychoneuroendocrinology, 29(7), 883-890. https://doi.org/10.1016/j.psyneuen.2003.08.001

Massey, J. M., Hubscher, C. H., Wagoner, M. R., Decker, J. A., Amps, J., Silver, J., \& Onifer, S. M. (2006). Chondroitinase ABC Digestion of the Perineuronal Net Promotes Functional Collateral Sprouting in the Cuneate Nucleus after Cervical Spinal Cord Injury. Journal of Neuroscience, 26(16), 4406-4414. https://doi.org/10.1523/JNEUROSCI.5467-05.2006

Matthews, R. T., Kelly, G. M., Zerillo, C. A., Gray, G., Tiemeyer, M., \& Hockfield, S. (2002). Aggrecan Glycoforms Contribute to the Molecular Heterogeneity of Perineuronal Nets. Journal of Neuroscience, 22(17), 7536-7547.

Matus, A., Delhaye-Bouchaud, N., \& Mariani, J. (1990). Microtubule-associated protein 2 (MAP2) in Purkinje cell dendrites: Evidence that factors other than binding to microtubules are involved in determining its cytoplasmic distribution. The Journal of Comparative Neurology, 297(3), 435-440. https://doi.org/10.1002/cne.902970308

McDonald, A. J., Hamilton, P. G., \& Barnstable, C. J. (2017). Perineuronal nets labeled by monoclonal antibody VC1.1 ensheath interneurons expressing parvalbumin and calbindin in the rat amygdala. Brain Structure and Function, 1-16. https://doi.org/10.1007/s00429-017-1542-8

McEchron, M. D., Tseng, W., \& Disterhoft, J. F. (2003). Single Neurons in CA1 Hippocampus Encode Trace Interval Duration during Trace Heart Rate (Fear) Conditioning in Rabbit. Journal of Neuroscience, 23(4), 1535-1547. https://doi.org/10.1523/JNEUROSCI.23-0401535.2003

Miller, M. J., Chen, N., Li, L., Tom, B., Weiss, C., Disterhoft, J. F., \& Wyrwicz, A. M. (2003). FMRI of the Conscious Rabbit during Unilateral Classical Eyeblink Conditioning Reveals 
Bilateral Cerebellar Activation. Journal of Neuroscience, 23(37), 11753-11758.

https://doi.org/10.1523/JNEUROSCI.23-37-11753.2003

Minta, K., Portelius, E., Janelidze, S., Hansson, O., Zetterberg, H., Blennow, K., \& Andreasson, U. (2019). Cerebrospinal Fluid Concentrations of Extracellular Matrix Proteins in Alzheimer's Disease. Journal of Alzheimer's Disease: JAD. https://doi.org/10.3233/JAD190187

Morikawa, S., Ikegaya, Y., Narita, M., \& Tamura, H. (2017). Activation of perineuronal netexpressing excitatory neurons during associative memory encoding and retrieval. Scientific Reports, 7, srep46024. https://doi.org/10.1038/srep46024

Morris, N. P., \& Henderson, Z. (2000). Perineuronal nets ensheath fast spiking, parvalbuminimmunoreactive neurons in the medial septum/diagonal band complex. European Journal of Neuroscience, 12(3), 828-838. https://doi.org/10.1046/j.14609568.2000.00970.x

Moyer, J. R., Power, J. M., Thompson, L. T., \& Disterhoft, J. F. (2000). Increased excitability of aged rabbit CA1 neurons after trace eyeblink conditioning. The Journal of Neuroscience: The Official Journal of the Society for Neuroscience, 20(14), 5476-5482.

Moyer, J. R., Thompson, L. T., \& Disterhoft, J. F. (1996). Trace eyeblink conditioning increases CA1 excitability in a transient and learning-specific manner. The Journal of Neuroscience: The Official Journal of the Society for Neuroscience, 16(17), 5536-5546.

Mueller, A. L., Davis, A., Sovich, S., Carlson, S. S., \& Robinson, F. R. (2016). Distribution of NAcetylgalactosamine-Positive Perineuronal Nets in the Macaque Brain: Anatomy and Implications. Neural Plasticity, 2016, 6021428. https://doi.org/10.1155/2016/6021428

Nabel, E. M., \& Morishita, H. (2013). Regulating Critical Period Plasticity: Insight from the Visual System to Fear Circuitry for Therapeutic Interventions. Frontiers in Psychiatry, 4. https://doi.org/10.3389/fpsyt.2013.00146

Nolan, B. C., Nicholson, D. A., \& Freeman, J. H. (2002). Blockade of GABAA Receptors in the Interpositus Nucleus Modulates Expression of Conditioned Excitation but not Conditioned Inhibition of the Eyeblink Response. Integrative Physiological and Behavioral Science: The Official Journal of the Pavlovian Society, 37(4), 293-310.

O'Connor, A. M., Burton, T. J., Mansuri, H., Hand, G. R., Leamey, C. A., \& Sawatari, A. (2019). Environmental Enrichment From Birth Impacts Parvalbumin Expressing Cells and Wisteria Floribunda Agglutinin Labelled Peri-Neuronal Nets Within the Developing Murine Striatum. Frontiers in Neuroanatomy, 13. https://doi.org/10.3389/fnana.2019.00090

Oh, M. M., \& Disterhoft, J. F. (2015). Increased Excitability of Both Principal Neurons and Interneurons during Associative Learning: The Neuroscientist. https://doi.org/10.1177/1073858414537382

Ohira, K., Takeuchi, R., Iwanaga, T., \& Miyakawa, T. (2013). Chronic fluoxetine treatment reduces parvalbumin expression and perineuronal nets in gamma-aminobutyric acidergic interneurons of the frontal cortex in adult mice. Molecular Brain, 6, 43. https://doi.org/10.1186/1756-6606-6-43

Paxinos, G., \& Watson, C. (2004). The Rat Brain in Stereotaxic Coordinates (5th ed.). Elsevier Academic Press, 2005. https://books-googlecom.www.libproxy.wvu.edu/books/about/The_Rat_Brain_in_Stereotaxic_Coordinates.ht ml?id=LKJqAAAAMAAJ

Perciavalle, V., Apps, R., Bracha, V., Delgado-García, J. M., Gibson, A. R., Leggio, M., Carrel, A. J., Cerminara, N., Coco, M., Gruart, A., \& Sánchez-Campusano, R. (2013). Consensus Paper: Current Views on the Role of Cerebellar Interpositus Nucleus in Movement Control and Emotion. The Cerebellum, 12(5), 738-757. https://doi.org/10.1007/s12311-013-0464-0 
Perea, G., Navarrete, M., \& Araque, A. (2009). Tripartite synapses: Astrocytes process and control synaptic information. Trends in Neurosciences, 32(8), 421-431. https://doi.org/10.1016/j.tins.2009.05.001

Person, A. L., \& Raman, I. M. (2012). Synchrony and neural coding in cerebellar circuits. Frontiers in Neural Circuits, 6, 97. https://doi.org/10.3389/fncir.2012.00097

Pizzorusso, T., Medini, P., Berardi, N., Chierzi, S., Fawcett, J. W., \& Maffei, L. (2002). Reactivation of Ocular Dominance Plasticity in the Adult Visual Cortex. Science, 298(5596), 1248-1251. https://doi.org/10.1126/science.1072699

Pugh, J. R., \& Raman, I. M. (2006). Potentiation of mossy fiber EPSCs in the cerebellar nuclei by NMDA receptor activation followed by postinhibitory rebound current. Neuron, 51(1), 113-123. https://doi.org/10.1016/j.neuron.2006.05.021

Rakic, P. (1971). Neuron-glia relationship during granule cell migration in developing cerebellar cortex. A Golgi and electonmicroscopic study in Macacus rhesus. Journal of Comparative Neurology, 141(3), 283-312. https://doi.org/10.1002/cne.901410303

Rowlands, D., Lensjø, K. K., Dinh, T., Yang, S., Andrews, M. R., Hafting, T., Fyhn, M., Fawcett, J. W., \& Dick, G. (2018). Aggrecan directs extracellular matrix mediated neuronal plasticity. Journal of Neuroscience, 1122-18. https://doi.org/10.1523/JNEUROSCI.112218.2018

Schreurs, B. G. (2019). Changes in cerebellar intrinsic neuronal excitability and synaptic plasticity result from eyeblink conditioning. Neurobiology of Learning and Memory, 166, 107094. https://doi.org/10.1016/j.nlm.2019.107094

Schreurs, B. G., Burhans, L. B., Smith-Bell, C. A., Mrowka, S. W., \& Wang, D. (2013). Ontogeny of trace eyeblink conditioning to shock-shock pairings in the rat pup. Behavioral Neuroscience, 127(1), 114-120. https://doi.org/10.1037/a0031298

Seeger, G., Brauer, K., Ha"rtig, W., \& Brückner, G. (1994). Mapping of perineuronal nets in the rat brain stained by colloidal iron hydroxide histochemistry and lectin cytochemistry. Neuroscience, 58(2), 371-388. https://doi.org/10.1016/0306-4522(94)90044-2

Shors, T. J., \& Miesegaes, G. (2002). Testosterone in utero and at birth dictates how stressful experience will affect learning in adulthood. Proceedings of the National Academy of Sciences of the United States of America, 99(21), 13955-13960. https://doi.org/10.1073/pnas.202199999

Slaker, M. L., Jorgensen, E. T., Hegarty, D. M., Liu, X., Kong, Y., Zhang, F., Linhardt, R. J., Brown, T. E., Aicher, S. A., \& Sorg, B. A. (2018). Cocaine Exposure Modulates Perineuronal Nets and Synaptic Excitability of Fast-Spiking Interneurons in the Medial Prefrontal Cortex. ENeuro, 5(5). https://doi.org/10.1523/ENEURO.0221-18.2018

Smith-Bell, C. A., Burhans, L. B., \& Schreurs, B. G. (2012). Predictors of susceptibility and resilience in an animal model of posttraumatic stress disorder. Behavioral Neuroscience, 126(6), 749-761. https://doi.org/10.1037/a0030713

Smith-Bell, C. A., \& Schreurs, B. G. (2017). Grouping subjects based on conditioning criteria reveals differences in acquisition rates and in strength of conditioning-specific reflex modification. Neurobiology of Learning and Memory, 145, 172-180. https://doi.org/10.1016/j.nlm.2017.10.004

Sorg, B. A., Berretta, S., Blacktop, J. M., Fawcett, J. W., Kitagawa, H., Kwok, J. C. F., \& Miquel, M. (2016). Casting a Wide Net: Role of Perineuronal Nets in Neural Plasticity. Journal of Neuroscience, 36(45), 11459-11468. https://doi.org/10.1523/JNEUROSCI.2351-16.2016

Stamenkovic, V., Stamenkovic, S., Jaworski, T., Gawlak, M., Jovanovic, M., Jakovcevski, I., Wilczynski, G. M., Kaczmarek, L., Schachner, M., Radenovic, L., \& Andjus, P. R. (2017). The extracellular matrix glycoprotein tenascin- $C$ and matrix metalloproteinases modify cerebellar structural plasticity by exposure to an enriched environment. Brain Structure \& Function, 222(1), 393-415. https://doi.org/10.1007/s00429-016-1224-y 
Stanton, M. E., Freeman, J. H., \& Skelton, R. W. (1992). Eyeblink conditioning in the developing rat. Behavioral Neuroscience, 106(4), 657-665. https://doi.org/10.1037/0735-

7044.106.4.657

Steinmetz, J. E., Lavond, D. G., \& Thompson, R. F. (1989). Classical conditioning in rabbits using pontine nucleus stimulation as a conditioned stimulus and inferior olive stimulation as an unconditioned stimulus. Synapse, 3(3), 225-233.

https://doi.org/10.1002/syn.890030308

Stryker, C., Camperchioli, D. W., Mayer, C. A., Alilain, W. J., Martin, R. J., \& MacFarlane, P. M. (2017). Respiratory dysfunction following neonatal sustained hypoxia exposure during a critical window of brainstem extracellular matrix formation. American Journal of Physiology. Regulatory, Integrative and Comparative Physiology, ajpregu.00199.2017. https://doi.org/10.1152/ajpregu.00199.2017

Sun, Z. Y., Bozzelli, P. L., Caccavano, A., Allen, M., Balmuth, J., Vicini, S., Wu, J.-Y., \& Conant, K. (2018). Disruption of perineuronal nets increases the frequency of sharp wave ripple events. Hippocampus, 28(1), 42-52. https://doi.org/10.1002/hipo.22804

Suttkus, A., Holzer, M., Morawski, M., \& Arendt, T. (2016). The neuronal extracellular matrix restricts distribution and internalization of aggregated Tau-protein. Neuroscience, 313(Supplement C), 225-235. https://doi.org/10.1016/j.neuroscience.2015.11.040

Thompson, R. F., \& Steinmetz, J. E. (2009). The role of the cerebellum in classical conditioning of discrete behavioral responses. Neuroscience, 162(3), 732-755.

https://doi.org/10.1016/j.neuroscience.2009.01.041

Tucker, R., Binder, L., Viereck, C., Hemmings, B., \& Matus, A. (1988). The sequential appearance of low- and high-molecular-weight forms of MAP2 in the developing cerebellum. The Journal of Neuroscience, 8(12), 4503-4512. https://doi.org/10.1523/JNEUROSCI.08-12-04503.1988

Umemori, J., Winkel, F., Castrén, E., \& Karpova, N. N. (2015). Distinct effects of perinatal exposure to fluoxetine or methylmercury on parvalbumin and perineuronal nets, the markers of critical periods in brain development. International Journal of Developmental Neuroscience: The Official Journal of the International Society for Developmental Neuroscience, 44, 55-64. https://doi.org/10.1016/j.ijdevneu.2015.05.006

Waddell, J., Mallimo, E., \& Shors, T. (2010). D-cycloserine reverses the detrimental effects of stress on learning in females and enhances retention in males. Neurobiology of Learning and Memory, 93(1), 31-36. https://doi.org/10.1016/j.nlm.2009.08.002

Wang, D., \& Schreurs, B. G. (2010). Dietary cholesterol modulates the excitability of rabbit hippocampal CA1 pyramidal neurons. Neuroscience Letters, 479(3), 327-331. https://doi.org/10.1016/j.neulet.2010.05.090

Wang, D., \& Schreurs, B. G. (2014). Maturation of membrane properties of neurons in the rat deep cerebellar nuclei. Developmental Neurobiology, 74(12), 1268-1276. https://doi.org/10.1002/dneu.22203

Wang, D., Smith-Bell, C. A., Burhans, L. B., O’Dell, D. E., Bell, R. W., \& Schreurs, B. G. (2018). Changes in membrane properties of rat deep cerebellar nuclear projection neurons during acquisition of eyeblink conditioning. Proceedings of the National Academy of Sciences, 115(40), E9419-E9428. https://doi.org/10.1073/pnas.1808539115

Wang, D., \& Zheng, W. (2015). Dietary cholesterol concentration affects synaptic plasticity and dendrite spine morphology of rabbit hippocampal neurons. Brain Research, 1622, 350360. https://doi.org/10.1016/j.brainres.2015.06.049

Welsh, J. P. (1992). Changes in the motor pattern of learned and unlearned responses following cerebellar lesions: A kinematic analysis of the nictitating membrane reflex. Neuroscience, 47(1), 1-19. https://doi.org/10.1016/0306-4522(92)90116-J

Xue, Y.-X., Xue, L.-F., Liu, J.-F., He, J., Deng, J.-H., Sun, S.-C., Han, H.-B., Luo, Y.-X., Xu, L.Z., Wu, P., \& Lu, L. (2014). Depletion of Perineuronal Nets in the Amygdala to Enhance 
the Erasure of Drug Memories. Journal of Neuroscience, 34(19), 6647-6658.

https://doi.org/10.1523/JNEUROSCI.5390-13.2014

Zheng, N., \& Raman, I. M. (2010). Synaptic inhibition, excitation, and plasticity in neurons of the cerebellar nuclei. Cerebellum (London, England), 9(1), 56-66. https://doi.org/10.1007/s12311-009-0140-6

Zheng, N., \& Raman, I. M. (2011). Prolonged Postinhibitory Rebound Firing in the Cerebellar Nuclei Mediated by Group I Metabotropic Glutamate Receptor Potentiation of L-Type Calcium Currents. Journal of Neuroscience, 31(28), 10283-10292.

https://doi.org/10.1523/JNEUROSCI.1834-11.2011 
Chapter 4. Doxycycline Alters Eyeblink Conditioning and the Perineuronal Net in Rat Deep Cerebellar Nuclei 


\subsection{Introduction}

The perineuronal net (PNN) is a specialized type of extracellular matrix surrounding fast spiking neurons in the central nervous system (Seeger et al., 1994; Celio et al., 1998; Carulli et al., 2006; Slaker et al., 2016; Sorg et al., 2016; Shen, 2018; Testa et al., 2018; Reichelt et al., 2019). The PNN has been implicated in playing many roles including but not limited to exit from the critical period of development as well as learning and memory. One way to easily manipulate the perineuronal net is to utilize the enzyme chondroitinase or chondroitinase $A B C(C h A B C)$. These enzymes have been infused directly into many brain regions, placed into electrophysiology baths, and virally expressed in order to alter the PNN (Massey et al., 2006; Gogolla et al., 2009; Xue et al., 2014; Chu et al., 2018; Hirono et al., 2018; Hettiaratchi et al., 2019; Anderson et al., 2020; Carulli et al., 2020; Lesnikova et al., 2021). Others have used transgenic techniques to prevent components of the PNN from being made or overexpressed enzymes more active in young animals into adulthood, keeping the critical period open (Weber et al., 1999; Bekku et al., 2012; Morawski et al., 2014; Miyata and Kitagawa, 2015, 2016; Hou et al., 2017; Yoshioka et al., 2017; Edamatsu et al., 2018; Wagner et al., 2020). While these tools are excellent ways to study the PNN, they may not be translationally feasible.

To overcome this hurdle, researchers have repurposed drugs in an attempt to achieve similar results to the pre-clinical research but in a more translational manner. Specifically, researchers have been investigating drugs already approved for use in humans including fluoxetine and doxycycline (Ohira et al., 2013, 2019; Guirado et al., 2014; Pollock et al., 2014; Umemori et al., 2015; Steinzeig et al., 2019; Puścian et al., 2021). Doxycycline is a tetracycline class antibiotic capable of crossing the blood brain barrier (Meli et al., 2006; Balducci et al., 2018; Schmidt et al., 2018; Balducci and Forloni, 2019). In addition to its function as an antibiotic, it can also act as an inhibitor of matrix metalloproteinases such as matrix metalloproteinase 9 
(Guimaraes et al., 2011; Bortolanza et al., 2018; Bach et al., 2019). MMP9 can cleave aggrecan and can act as a gelatinase. It is also highly associated with neuronal changes related to learning and memory function. There is some interesting work suggesting that doxycycline can potentially alter memory storage or consolidation (Bach et al., 2017, 2019). The theory underlying how doxycycline would work is that by inhibiting the activity of MMP9, it could potentially prevent some remodeling of the PNN. Evidence suggests that the PNN undergoes digestion during acquisition phases of learning but returns to relatively normal levels during consolidation (Carulli et al., 2020). By blocking the initial digestion of the PNN, can we disrupt the normal process of learning?

One of the goals of this research is to determine if the daily administration of doxycycline has any influence on the acquisition, extinction, or retention of EBC in adult male and female rats. The animals were given a dose of $40 \mathrm{mg} / \mathrm{kg}$ doxycycline 2 hours prior to behavior, allowing time for the drug to cross the blood brain barrier. 


\subsection{Methods:}

\subsubsection{Animals}

Seventeen Long Evans rats (Rattus norvegicus) (9 F, 8 M) at 3 - 6 months of age were supplied by Charles River (Wilmington, MA). Eleven rats underwent surgery, intraperitoneal (IP) injections, and eyeblink conditioning. Rats were housed with cagemates of the same sex after weaning and were given ad libitum access to food and water and maintained on a $12 \mathrm{~h}$ light/dark cycle, all in accordance with the National Institute of Health guidelines. Adult rats undergoing electromyogram (EMG) surgery were housed individually post-surgery. This was to prevent damage to the EMG that results from social grooming. All procedures were approved by the West Virginia University (WVU) Animal Care and Use Committee and the WVU Biosafety Committee.

\subsubsection{Surgeries}

Fifteen 3 - 6-month-old rats (7 F, $8 \mathrm{M})$ were fitted with the EMG hardware for eyeblink conditioning. Sterile surgical technique was used. Each rat was sedated with isoflurane ( $5 \%$ induction, $2 \%$ maintenance) with supplemental oxygen (0.5 -1 L/min). The incision site (scalp) was shaved and washed three times with alternating swipes of Povidone-iodine scrub and $70 \%$ alcohol followed by application of Povidone-iodine solution after which the rat was positioned in a rodent adult stereotaxic (Kopf Model 900LS) device. Bupivacaine (max dose 2mg/kg), a local anesthetic, was infiltrated into the scalp, which was incised with a surgical scalpel blade, to create an approximately $20-\mathrm{mm}$ incision, just rostral to the eyes and just caudal to the ears. The scalp was reflected and cleaned, exposing just enough of the skull to accommodate a plastic connector for the EMG implant. Holes were drilled into the skull for skull screws. Gelfoam (Capital Wholesale Drug Company, 245780) was used to stop any bleeding. The rats were next fitted with differential EMG electrodes that were implanted in the left eyelid muscle by inserting a sterile 25-gauge needle (affixed to a 1-mm empty sterile syringe) through the eyelid until the needle tip was visible 
on the other side as a result of the open scalp incision. The needle was then threaded with the fine wire EMG electrodes and pulled through the eyelid, leaving the recording electrode in the middle of the eyelid and the reference wire in the rostral corner of the eyelid. Where in contact around the eyelid, wires were stripped of insulation and excess wire was trimmed in an effort to prevent displacement via grooming. A bipolar stimulating electrode for delivering the electrical stimulation unconditioned stimulus was implanted subdermally, immediately caudal to the eye. The EMG recording electrode and stimulating electrode wire leads terminated on gold pins in a plastic connector, which was secured to the top of the skull and the skull screws with acrylic dental cement. The surgical site was closed with nylon suture on both sides of the plastic connector. A non-steroidal anti-inflammatory analgesic (ketoprofen $1 \mathrm{mg} / \mathrm{mL}$ ) was administered at the end of surgery and $3 \mathrm{~mL}$ of lactated Ringer's solution was given subcutaneously to restore any fluids lost during surgery.

\subsubsection{Eyeblink Conditioning}

Rats were allowed to recover from EMG surgery for three days. On the fourth day after surgery, they underwent an equipment test and were weighed. The rats were also given a brief $10 \mathrm{~min}$ habituation period to accustom them to the head plug, cable, and modular test cage. The following day, acquisition began, it consisted of one session per day for five days of paired tone conditioned stimulus (CS) and periorbital electrical stimulation unconditioned stimulus (US) training followed by one session per day for three days of CS-alone extinction. The rat head plug was connected to a cable connected to a freely-rotating commutator which allowed the rat to move freely around a Coulburn Instruments modular testing cage within a sound-attenuating training chamber. The back wall of the chamber had a panel containing a speaker mounted at a $45^{\circ}$ angle above the testing cage. The cable separated and terminated as the input to an AC/DC differential EMG amplifier (A-M Systems, Sequim, WA) and the output of a stimulus isolator (World Precision Instruments (WPI), Sarasota, FL) for shock delivery. The training chamber was lit with 
an LED and contained a low-light camera to allow videographic monitoring of the animal at all times. Shock was delivered by a rechargeable, constant-current stimulator (WPI, Model A365) that had been calibrated with a multimeter (WPI). LabVIEW software (National Instruments, Austin, TX) controlled the delivery of stimuli and the recording of eyelid EMG activity. Each adult rat was adapted to the enclosure without stimulus presentations for $10 \mathrm{~min}$ before every session. Paired delay conditioning sessions consisted of 100 trials each with 90 paired presentations of a tone CS and a periorbital electrical stimulation US as well as 10 tone-alone test trials or probes presented after every ninth paired trial to assess integrated EMG activity without a shock artifact. The tone CS consisted of a $380-\mathrm{ms}, 88-\mathrm{db}, 2.8-\mathrm{kHz}$ pure tone. The electrical stimulation US consisted of a $100-\mathrm{ms}, 3.0-4.0-\mathrm{mA}, 60-\mathrm{Hz}, \pm 50$-volt, square-wave constant-current pulse to the periorbital region using the pre-calibrated stimulator. A blanking circuit in operation during the US prevented the shock from swamping the EMG signal. During the tone-shock paired trials, the CS co-terminated with the US. During acquisition, all trials were separated by an inter-trial interval averaging $30 \mathrm{~s}$. To assess memory of and to extinguish responding to the tone CS, we performed CS-alone extinction beginning one day after the last paired training session. The tone CS was presented by itself for 100 trials daily on each of three consecutive days. Every $10^{\text {th }}$ trial during extinction was considered a probe trial. The rats were weighed immediately prior to the equipment test, sessions 2 and 4 of acquisition, and the first extinction session.

\subsubsection{Behavior Data Analysis}

Data analyses have been described previously (Schreurs et al., 2013; Smith-Bell and Schreurs, 2017; Wang et al., 2018; O'Dell et al., 2020). In brief, EMG signal was filtered (300 - 3,000-Hz), amplified, and stored (raw EMG), in addition to being rectified and integrated (20-ms time constant). Baseline activity was averaged during the first $200-\mathrm{ms}$ from trial onset. If EMG activity 100-ms before CS onset was four standard deviations (SDs) or more above baseline, the trial was omitted from analysis to ensure that movement or spontaneous blinking artifacts did not 
artificially inflate response levels $(\sim 1 \%$ of total trials). During paired trials, we characterized unconditioned responses (URs) to the US as integrated EMG activity occurring 35-ms after the end of the US that exceeded the average baseline value by eight SDs. CRs were assessed as EMG activity that exceeded 8 SDs above the baseline during the period 80 -ms after tone onset until just before US onset. This window ensured that US signal and movement or startle artifacts did not artificially inflate levels of responding. During probe trials, CRs were assessed as EMG activity beginning $80-\mathrm{ms}$ after CS onset, to eliminate the potential for including alpha responses, that was eight SDs above the average baseline value during a $200-\mathrm{ms}$ pre-CS period. In addition to conditioned response frequency indicated as \%CRs, the amplitude of each response was calculated as the average EMG signal during the baseline period, subtracted from the maximum EMG signal during the response period. Area under the curve of the CR was obtained by summing the average heights of 2.5-ms bins of data that occurred during the CR period. Peak latency of the $\mathrm{CR}$ was calculated as the time when the maximum CR period height occurred (Schreurs et al., 2013; Wang et al., 2018). If an animal did not reach $70 \%$ CRs during probe trials on at least one day of acquisition, they were excluded from behavioral analyses. This is the laboratory's standard for determining adequate levels of EBC in rats for a determination of conditioning-specific changes in unconditioned responding.

\subsubsection{Doxycycline mediated manipulation of the PNN}

A $40 \mathrm{mg} / \mathrm{kg}$ doxycycline solution or vehicle (0.9\% USP Saline) was given as an IP injection two hours prior to EBC. Rats were given the injection in their housing room and were returned to their home cage until training. The dosage and timing of doxycycline was derived from the literature (Guimaraes et al., 2011; El-Neweshy, 2013; Wang et al., 2014; Hecht et al., 2016).

Cagemates were randomly assigned to the doxycycline or vehicle group following the equipment test and attempts were made to ensure equal numbers of males and females were included in each group. 


\subsubsection{Tissue Processing}

Rats used for the doxycycline or saline IP injections and eyeblink conditioning were given a subcutaneous injection of ketamine hydrochloride $(80 \mathrm{mg} / \mathrm{kg})$ mixed with xylazine $(8.0 \mathrm{mg} / \mathrm{kg})$. Animals were perfused transcardially with $0.9 \%$ saline ( $\mathrm{pH} 7.4$ at room temperature) followed by 4\% paraformaldehyde. Brains were collected and placed in fixative until ready for processing. Brains were transferred to $30 \%$ sucrose for cryoprotection until they sank, then $40-\mu \mathrm{m}$ sections were cut on a freezing microtome. Tissue was either mounted onto $3 \%$ gelatin slides or placed into cryoprotection at $-20^{\circ} \mathrm{C}$.

\subsubsection{PNN immunofluorescence}

Mounted sections of cerebellar tissue were washed in $0.1 \mathrm{M}$ phosphate buffered saline (PBS) $1 \%$ Tween then blocked for $2 \mathrm{~h}$ with $5 \%$ normal donkey serum or normal goat serum and $3 \% \mathrm{BSA}$ before being incubated with primary antibodies overnight at $4^{\circ} \mathrm{C}$. Following washing in $0.1 \mathrm{MPBS}$, sections were incubated in secondary antibody and $1 \%$ blocking solution for $4 \mathrm{~h}$. After completing the secondary antibody reaction, sections were washed again in $0.1 \mathrm{M}$ PBS. Following the final wash, sections were cover-slipped with DAPI Fluoromount-G mounting media and \#1.5 coverslips (Fisher Scientific). Manufacturers' information for primary antibodies, secondary antibodies, and sera can be found in Table 1.

Table 4.1. Materials used in Tissue Processing

\begin{tabular}{|l|}
\hline Item (manufacturer) \\
\hline Doxycycline Hyclate (Millipore Sigma, cat\#: 1226003-200MG) \\
\hline Biotin conjugated wisteria floribunda lectin (EY Laboratories Inc., cat\#: BA-3101-1) (1:1000) \\
\hline Anti-MAP2, clone AP20 (EMD Millipore, cat\#: MAB3418) (1:200) \\
\hline Donkey Anti-goat A488 (Thermofisher Scientific Inc., Invitrogen, cat\#: A-11055) (1:500) \\
\hline Goat Anti-mouse A488 (Thermofisher Scientific Inc., Invitrogen, cat\#: A-11029) (1:500) \\
\hline
\end{tabular}


Streptavidin, Alexa Fluor 647 conjugate (Thermofisher Scientific Inc., Invitrogen, cat\#: S21374) (1:500)

Normal donkey serum (Millpore Sigma, cat: S30-100KC)

Normal goat serum (Thermofisher Scientific Inc., Gibco, cat\#: PCN5000)

Bovine serum albumin (Millipore Sigma, cat\#: A3294)

DAPI Fluoromount G (SouthernBiotech, cat\#: 0100-20)

\subsubsection{Image Acquisition}

Neuronal and PNN immunofluorescent reactivity in the rat cerebellum was visualized using a confocal laser-scanning microscope (Zeiss LSM 710; Carl Zeiss International). Images were acquired using 405, 488, 546, and $633 \mathrm{~nm}$ lasers, sequential multichannel line scan, and with filters set manually to detect the spectral peak of each fluorophore. Cells were imaged at 20X (NA 0.4) and 63X oil-immersion (NA 1.4) objectives. Raw images were exported to Zen Lite 2009 and Adobe Photoshop CC 2017 to make minor adjustments to the brightness and contrast. Raw images were also exported to FIJI (ImageJ, NIH) to determine the percentage of WFA positive (WFA+) neurons (neurons with a PNN. For PNN analysis, an average of 6 sections per animal were imaged; images were analyzed between the following coordinates (Bregma: 11.52mm, Interaural: $-2.52 \mathrm{~mm}$ and Bregma: $10.68 \mathrm{~mm}$, Interaural: $-1.68 \mathrm{~mm}$ ). Each section was imaged bilaterally with the imager blind to the side. In FIJI, the channels were separated in order to compare the number of microtubule-associated protein 2-positive (MAP2+) cells to the WFA+ cells to obtain a normalized count for each image. MAP2 is a protein found in both developing and adult neurons and can be used as a biomarker for neurons (Tucker et al., 1988; Matus et al., 1990; Kindler and Garner, 1994; Dehmelt and Halpain, 2004). Once channels were separated, a background subtraction was performed to further distinguish WFA+ or MAP2+ cells. Images were thresholded to produce binary images and then particle analysis was used to generate a count of 
WFA+ or MAP2+ cells. Counts were made every ten slices and the average number of WFA+ cells divided by the average number of MAP2+ cells and then multiplied by 100 to generate a percentage of PNN+ neurons.

\subsubsection{Statistical Analysis}

Data are presented as mean and \pm SEM. Two-way ANOVA and unpaired t-tests were calculated in IBM SPSS Statistics (Ver.26.00.0; IBM Corp.) with $p<0.05$ as the criterion for significance. 


\subsection{Results}

\subsubsection{Doxycycline may prevent modulation of the PNN in the AIN}

\subsubsection{Acute Doxycycline}

Injections

We examined WFA reactivity of MAP2+ cells in the AIN of two adult rats acutely 24 hours after doxycycline IP injection and
A

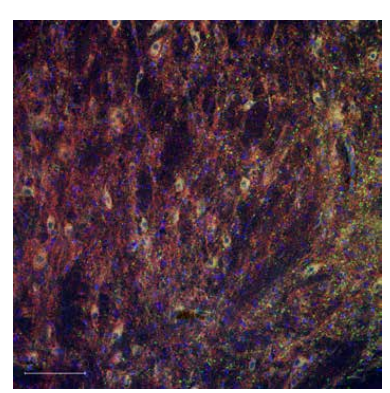

B

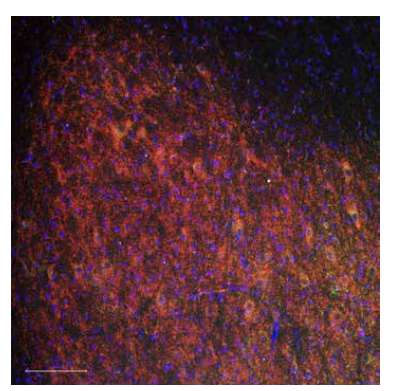

C

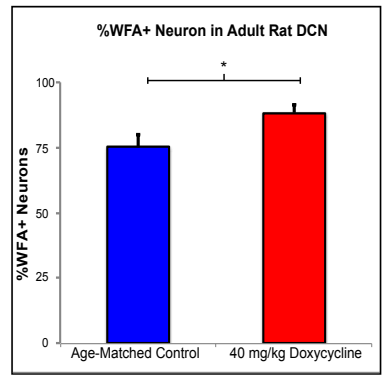

Figure 4.1. Acute Doxycycline Injections.

An acute injection of doxycycline may prevent remodeling of the PNN. ${ }^{*} p<0.05$, Error bars SEM.

animals given doxycycline had higher \% of WFA reactivity, $(88.31 \pm 3.24 \%)$ compared to the animals receiving vehicle $(75.78 \pm 4.69 \%), p=.031$. These data are found in Figure 4.1.

\subsubsection{Daily Doxycycline Injections prior to EBC}

Figure 4.2 shows that this is also the case following EBC and experiments. We attempted to block PNN remodeling by performing IP injections 2 hours prior to EBC of either doxycycline or vehicle. The PNN in the AIN in the animals that received the doxycycline injections was higher based on WFA reactivity $(87.85 \pm 2.34 \%)$ compared to the animals receiving vehicle $(74.85 \pm$ $4.8 \%), p=.041$

\subsubsection{Doxycycline does not alter} Eyeblink Conditioning

\subsubsection{EBC Acquisition}

Rats were removed from the study if their EMG signal-tonoise ratio was so small it was impossible to distinguish their

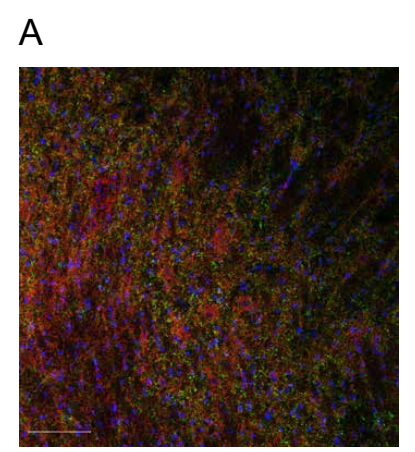

B

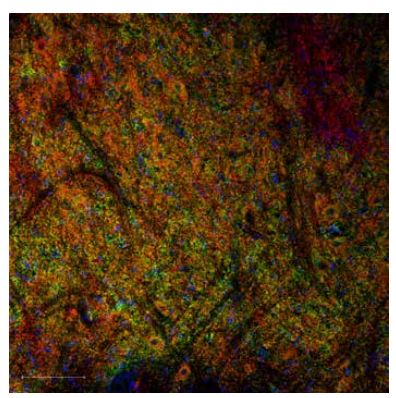

C

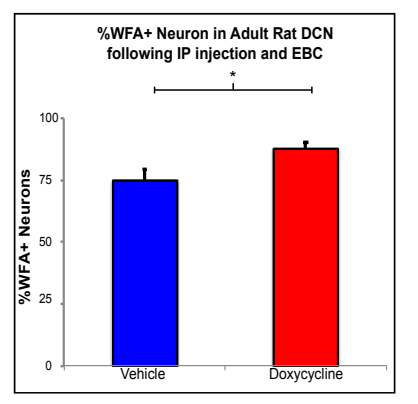

Figure 4.2. Doxycycline or Vehicle Injections and WFA reactivity.

An acute injection of doxycycline may prevent remodeling of the PNN following EBC.

${ }^{*} p<0.05$, Error bars SEM. 
responses from baseline activity, four rats were removed due to poor signal. Eleven adult male and female rats (at least P90) were included in the behavioral data analysis after five sessions of paired EBC ( $n=6$ doxy (3F,3M), $n=4$ vehicle $(2 F, 2 M)$ on final acquisition session), up to three sessions of tone-only extinction $(n=7$ doxy $(3 F, 4 M), n=4$ vehicle $(2 F, 2 M)$ on final extinction session) and one day of retention $(n=7$ doxy $(3 F, 4 M), n=4$ vehicle $(2 F, 2 M))$. One male animal in the doxy group lost signal during the final two sessions of acquisition but signal improved during the extinction sessions. This is one reason why even if an animal had poor EMG signal and their behavioral results could not be analyzed for that particular day, they were still placed in the chamber and exposed to the stimuli and brain tissue was stained and imaged for PNN analysis.

Figure 4.3 shows there was an increase in \%CRs in both paired and probe trials for both groups across sessions, [F $(4,43)=6.71, p<.000]$, with Session $1(\mathrm{~S} 1)$ having lower \%CRs on paired trials than S2 $(p=.037), S 3(p=.006), S 4(p<.000)$, and S5 $(p<.000) . S 5$ had higher \%CRs than S2

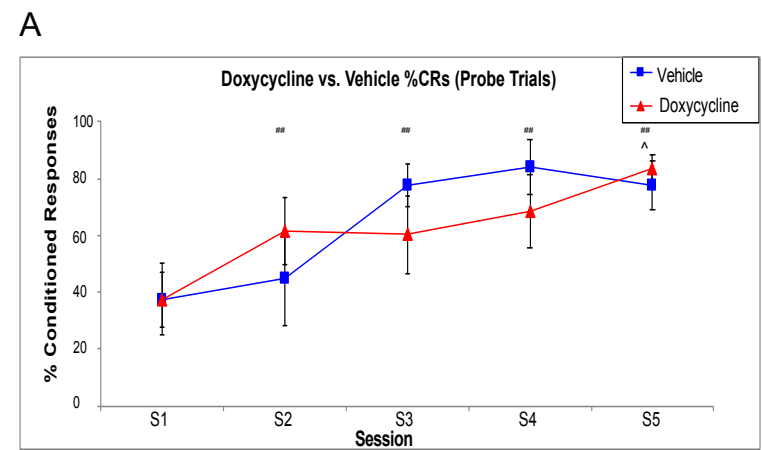

B

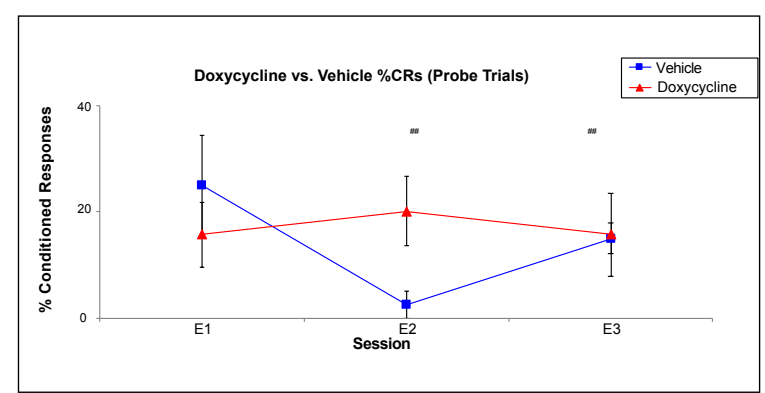

Figure 4.3. Percent Conditioned Responses between Doxycycline and Vehicle Rats. A. There were no differences in \%CRs between the two groups but there were sessions differences, showing both groups are capable of learning EBC. B. We noted differences between sessions during extinction. \#\# = p $<.05$ for session

$(p=.008)$ and S3 $(p=.045)$. Similar session differences were also observed on probe trials as well, $[F(4,43)=4.36, p=.005]$, with Session $1(\mathrm{~S} 1)$ having lower \%CRs on probes than S3 $(p=$ $.015)$, S4 $(p=.003)$, and S5 ( $p=.001)$. S2 also had lower \%CRs than S5 $(p=.035)$. However, there were no overall differences in \%CRs between groups on either paired or probe trials. 
We noted changes to some parameters of CRs during paired EBC, which are important tools for measuring an animal's ability to learn EBC. There were session differences associated with peak eyeblink latency on probe trials only, [F (4, $41)=3.44, p=.016]$ with the eyeblink peak response occurring earlier on S4 $(p=.004)$, and S5 $(p=.031)$ compared to S1 (Smith-Bell et al., 2012) seen in Figure 4.4. The eyeblink response grew faster by the

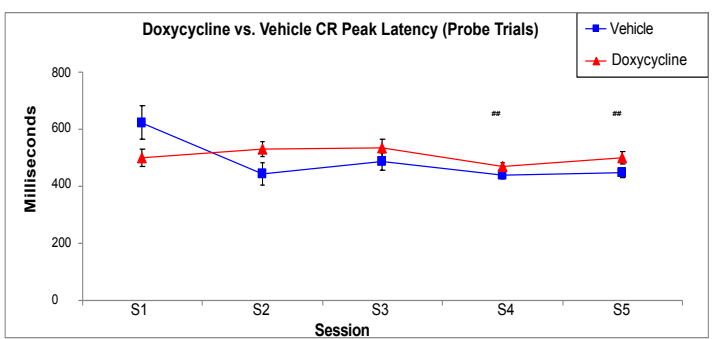
end of acquisition. There was a group by session interaction in the peak latency of the CR, $[F(4,41)=$ 3.28, $p=.020$ ] likely driven by the session Figure 4.4. Peak Latency of Conditioned Responses between Doxycycline and Vehicle Rats during Acquisition. There were session differences in timing of the CR with responses speeding up. \#\# = p $<.05$ for session

differences.

We did not notice any session differences in the area or the amplitude of the eyeblink response $(\mathrm{F}$ 's $<1)$. However, the doxycycline rats group had reduced $\mathrm{CR}$ area compared to the vehicle group in both paired, $[F(1,43)=5.36, p=.025]$ and probe trials $[F(1,43)=6.05, p=$ .018] as seen in Figure 4.5.

\subsubsection{EBC Extinction}


Figure 4.3 shows the \%CRs during the three extinction sessions. Analysis yielded session differences of paired trials, $[F(2,27)=5.43, p=$ .010], with the first extinction session (E1) having lower \%CRs than the second $(E 2)(p=.015)$ and the final extinction session $(E 3)(p=.012)($ Fig 4B).

A

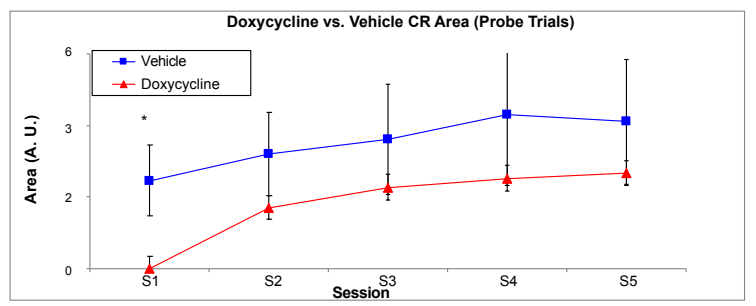

B

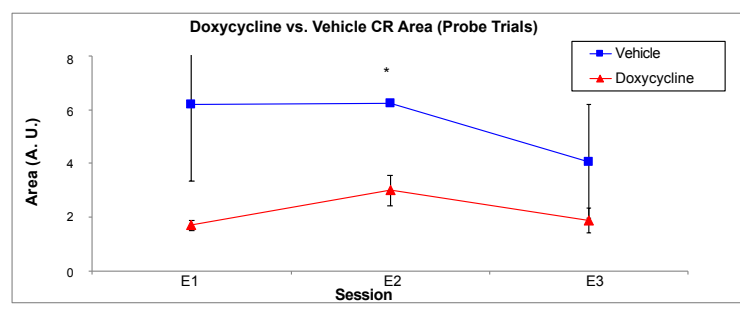

Figure 4.5. Area of Conditioned Responses between Doxycycline and Vehicle Rats. There were no session differences in CR area groups but there were group differences, showing vehicle rats had a higher $C R$ area during $A$. acquisition and $B$. extinction. ${ }^{*}=p<.05$ for group

\subsubsection{Unconditioned Response}

The foregoing changes we found in CR area may have been contributed to by significant differences found in the UR. The \%URs between the groups were different, $[F(1,43)=15.58, p$ $<.000$ ], with rats given doxycycline having much more URs than the vehicle group found in Figure 4.6. In addition, there were also group differences on the UR area, $[F(1,43)=8.44, p=.006]$, but the vehicle animals have a larger area of the UR seen in Figure 4.6. Even though the doxycycline injected rats were responding more often to the UR, their responses were still smaller in area compared to the vehicle group. These results suggest that the behavioral differences between the two groups may be related to non-associative effects sensitizing the doxycycline animals to our US. 


\subsection{Discussion}

In summary, we found that male and female rats given a dose of doxycycline had more WFA reactivity in the DCN. These animals were capable of learning and extinguishing EBC. However, any group effects may have been the result of non-associative effects of doxycycline.

Although we did observe different levels of WFA reactivity between groups, the changes were not as marked as we previously observed. We expected that doxycycline would prevent learning-related modulation of the PNN. We do observe what appears to be a greater reduction of the PNN in vehicle animals, which may be related to learning. Other researchers have noted that animals who have undergone learning-related training have less WFA reactivity compared to their naïve counterparts. However, our doxycycline animals were capable of learning and retaining $\mathrm{EBC}$, suggesting some amount of

remodeling took place. A more selective MMP9 inhibitor could be employed to investigate if learning could be blocked entirely. We also did following behavior. This is a logical next step to determine the actual amount of MMP9 activity.

We did not observe as many group differences in EBC as we did in our previous work with $\mathrm{ChABC}$. These changes are likely related to the how ChABC acts comparatively to doxycycline's proposed method. Doxycycline
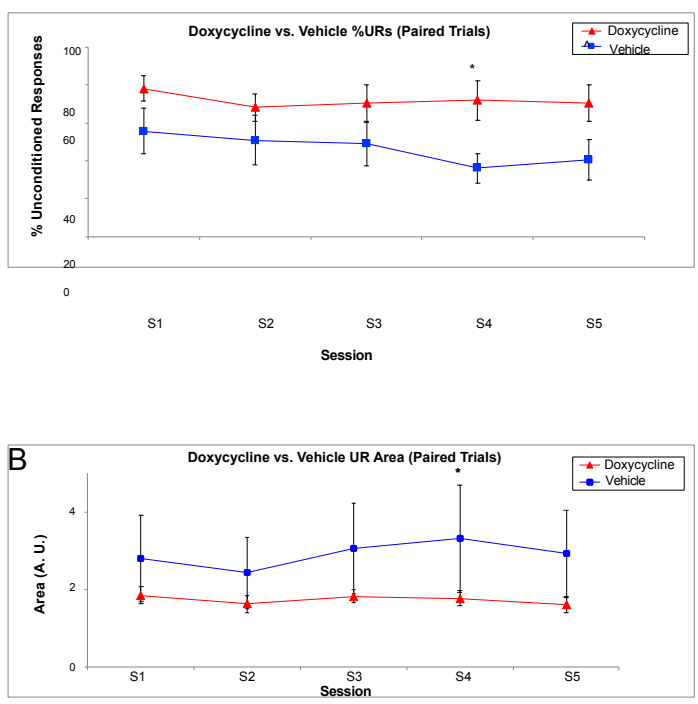

Figure 4.6. Unconditioned Responses between Doxycycline and Vehicle Rats. A. UR differences were observed with doxycycline rats having more UR than vehicle. B. There were group differences in UR area groups, showing vehicle rats had a higher UR area during acquisition. ${ }^{*}=p<.05$ for group

would prevent the modification of the PNN while ChABC drastically reduces the PNN in 
comparison. Even among ChABC studies, the level of digestion influences behavior with the most pronounced changes occurring with the greatest reduction of PNN. However, even modest levels of digestion can have effects on animal behavior. These changes also could be the result of the non-associative effects we observed in comparison to ChABC. We found no evidence of UR differences when using $\mathrm{ChABC}$ nor have there been reports of such in the literature. Another avenue of exploration would be to alter the method of introducing doxycycline. Potentially using oral gavage or adding doxycycline to the water supply could produce different effects than IP. Additionally, we have encountered substances that have altered the CR and UR of EBC previously albeit in the rabbit (Wang et al., 2006). To examine the effects of 4-aminopyridine (4AP) , we also included unpaired controls. In order to determine if 4-AP was sensitizing the rabbits to the tone, the US, or both, the animals were given tones of increasing intensity as well as different intensities of the US. These methods should reveal a more complete idea of how doxycycline is altering the UR.

Another potential future experiment would be to investigate the electrophysiological changes, if any, that could be taking place. We were unable to perform these experiments in the adult animal as it is incredibly difficult to successfully patch and record from adult DCN cells. Shifting the age of the experiment down to P23-24 would overcome this difficulty. We have previous experience performing EBC and electrophysiological experiments in animals of this age (Wang et al., 2018; O’Dell et al., 2020).

\subsection{Conclusions}

Although these results may be frustrating, they do allow us to gain valuable information regarding doxycycline as a potential method of manipulating the PNN. In addition to considerations of dose, route of administration could also be playing a role. Further investigation of the actual changes to MMP-9 should also be carried out to determine how much activity the current dosage is suppressing. Increased or prolonged dosage of daily doxycycline could be difficult to perform 
as it can cause fatal effects in rats, leading to cardiac failure but could be an option going forward. Continuing this line of inquiry will hopefully solidify the unanswered questions. 


\section{References:}

Anderson MD, Paylor JW, Scott GA, Greba Q, Winship IR, Howland JG (2020) ChABC infusions into medial prefrontal cortex, but not posterior parietal cortex, improve the performance of rats tested on a novel, challenging delay in the touchscreen TUNL task. Learn Mem Cold Spring Harb N 27:222-235.

Bach DR, Näf M, Deutschmann M, Tyagarajan SK, Quednow BB (2019) Threat Memory Reminder Under Matrix Metalloproteinase 9 Inhibitor Doxycycline Globally Reduces Subsequent Memory Plasticity. J Neurosci 39:9424-9434.

Bach DR, Tzovara A, Vunder J (2017) Blocking human fear memory with the matrix metalloproteinase inhibitor doxycycline. Mol Psychiatry Available at: http://www.nature.com/mp/journal/vaop/ncurrent/full/mp201765a.html?foxtrotcallback=tru e [Accessed August 15, 2017].

Balducci C, Forloni G (2019) Doxycycline for Alzheimer's Disease: Fighting $\beta$-Amyloid Oligomers and Neuroinflammation. Front Pharmacol 10 Available at: https://www.frontiersin.org/articles/10.3389/fphar.2019.00738/full [Accessed August 5, 2019].

Balducci C, Santamaria G, La Vitola P, Brandi E, Grandi F, Viscomi AR, Beeg M, Gobbi M, Salmona M, Ottonello S, Forloni G (2018) Doxycycline counteracts neuroinflammation restoring memory in Alzheimer's disease mouse models. Neurobiol Aging 70:128-139.

Bekku Y, Saito M, Moser M, Fuchigami M, Maehara A, Nakayama M, Kusachi S, Ninomiya Y, Oohashi T (2012) Bral2 is indispensable for the proper localization of brevican and the structural integrity of the perineuronal net in the brainstem and cerebellum. J Comp Neurol 520:1721-1736.

Bortolanza M, Nascimento GC, Socias SB, Ploper D, Chehín RN, Raisman-Vozari R, Del-Bel E (2018) Tetracycline repurposing in neurodegeneration: focus on Parkinson's disease. J Neural Transm Available at: https://doi.org/10.1007/s00702-018-1913-1 [Accessed August 21, 2018].

Carulli D, Broersen R, de Winter F, Muir EM, Mešković M, de Waal M, de Vries S, Boele H-J, Canto CB, De Zeeuw Cl, Verhaagen J (2020) Cerebellar plasticity and associative memories are controlled by perineuronal nets. Proc Natl Acad Sci U S A.

Carulli D, Rhodes KE, Brown DJ, Bonnert TP, Pollack SJ, Oliver K, Strata P, Fawcett JW (2006) Composition of perineuronal nets in the adult rat cerebellum and the cellular origin of their components. J Comp Neurol 494:559-577.

Celio MR, Spreafico R, Biasi SD, Vitellaro-Zuccarello L (1998) Perineuronal nets: past and present. Trends Neurosci 21:510-515.

Chu P, Abraham R, Budhu K, Khan U, De Marco Garcia N, Brumberg JC (2018) The Impact of Perineuronal Net Digestion using Chondroitinase ABC on the Intrinsic Physiology of Cortical Neurons. Neuroscience.

Dehmelt L, Halpain S (2004) The MAP2/Tau family of microtubule-associated proteins. Genome Biol 6:204.

Edamatsu M, Miyano R, Fujikawa A, Fujii F, Hori T, Sakaba T, Oohashi T (2018) Hapln4/Bral2 is a selective regulator for formation and transmission of GABAergic synapses between Purkinje and deep cerebellar nuclei neurons. J Neurochem 147:748-763.

El-Neweshy MS (2013) Experimental doxycycline overdose in rats causes cardiomyopathy. Int J Exp Pathol 94:109-114.

Gogolla N, Caroni P, Lüthi A, Herry C (2009) Perineuronal nets protect fear memories from erasure. Science 325:1258-1261.

Guimaraes DA, Rizzi E, Ceron CS, Oliveira AM, Oliveira DM, Castro MM, Tirapelli CR, Gerlach RF, Tanus-Santos JE (2011) Doxycycline Dose-dependently Inhibits MMP-2-Mediated Vascular Changes in 2K1C Hypertension. Basic Clin Pharmacol Toxicol 108:318-325. 
Guirado R, Perez-Rando M, Sanchez-Matarredona D, Castrén E, Nacher J (2014) Chronic fluoxetine treatment alters the structure, connectivity and plasticity of cortical interneurons. Int J Neuropsychopharmacol Oxf 17:1635-1646.

Hecht E, Freise C, Websky KV, Nasser H, Kretzschmar N, Stawowy P, Hocher B, Querfeld U (2016) The matrix metalloproteinases 2 and 9 initiate uraemic vascular calcifications. Nephrol Dial Transplant Off Publ Eur Dial Transpl Assoc - Eur Ren Assoc 31:789-797.

Hettiaratchi MH, O'Meara MJ, Teal CJ, Payne SL, Pickering AJ, Shoichet MS (2019) Local delivery of stabilized chondroitinase $A B C$ degrades chondroitin sulfate proteoglycans in stroke-injured rat brains. J Controlled Release 297:14-25.

Hirono M, Watanabe S, Karube F, Fujiyama F, Kawahara S, Nagao S, Yanagawa Y, Misonou H (2018) Perineuronal nets in the deep cerebellar nuclei regulate GABAergic transmission and delay eyeblink conditioning. J Neurosci:3238-17.

Hou X, Yoshioka N, Tsukano H, Sakai A, Miyata S, Watanabe Y, Yanagawa Y, Sakimura K, Takeuchi K, Kitagawa H, Hensch TK, Shibuki K, Igarashi M, Sugiyama S (2017) Chondroitin Sulfate Is Required for Onset and Offset of Critical Period Plasticity in Visual Cortex. Sci Rep 7:12646.

Kindler S, Garner CC (1994) Four repeat MAP2 isoforms in human and rat brain. Brain Res Mol Brain Res 26:218-224.

Lesnikova A, Casarotto PC, Fred SM, Voipio M, Winkel F, Steinzeig A, Antila H, Umemori J, Biojone C, Castrén E (2021) Chondroitinase and Antidepressants Promote Plasticity by Releasing TRKB from Dephosphorylating Control of PTPo in Parvalbumin Neurons. J Neurosci 41:972-980.

Massey JM, Hubscher CH, Wagoner MR, Decker JA, Amps J, Silver J, Onifer SM (2006) Chondroitinase ABC Digestion of the Perineuronal Net Promotes Functional Collateral Sprouting in the Cuneate Nucleus after Cervical Spinal Cord Injury. J Neurosci 26:44064414.

Matus A, Delhaye-Bouchaud N, Mariani J (1990) Microtubule-associated protein 2 (MAP2) in Purkinje cell dendrites: evidence that factors other than binding to microtubules are involved in determining its cytoplasmic distribution. J Comp Neurol 297:435-440.

Meli DN, Coimbra RS, Erhart DG, Loquet G, Bellac CL, Täuber MG, Neumann U, Leib SL (2006) Doxycycline Reduces Mortality and Injury to the Brain and Cochlea in Experimental Pneumococcal Meningitis. Infect Immun 74:3890-3896.

Miyata S, Kitagawa H (2015) Mechanisms for modulation of neural plasticity and axon regeneration by chondroitin sulphate. J Biochem (Tokyo) 157:13-22.

Miyata S, Kitagawa H (2016) Chondroitin 6-Sulfation Regulates Perineuronal Net Formation by Controlling the Stability of Aggrecan. Neural Plast 2016 Available at: https://www.hindawi.com/journals/np/2016/1305801/abs/ [Accessed January 9, 2017].

Morawski M, Dityatev A, Hartlage-Rübsamen M, Blosa M, Holzer M, Flach K, Pavlica S, Dityateva G, Grosche J, Brückner G, Schachner M (2014) Tenascin-R promotes assembly of the extracellular matrix of perineuronal nets via clustering of aggrecan. Phil Trans R Soc B 369:20140046.

O'Dell DE, Schreurs BG, Smith-Bell C, Wang D (2020) Disruption of rat deep cerebellar perineuronal net alters eyeblink conditioning and neuronal electrophysiology. Neurobiol Learn Mem 177:107358.

Ohira K, Hagihara H, Miwa M, Nakamura K, Miyakawa T (2019) Fluoxetine-induced dematuration of hippocampal neurons and adult cortical neurogenesis in the common marmoset. Mol Brain 12:69.

Ohira K, Takeuchi R, Iwanaga T, Miyakawa T (2013) Chronic fluoxetine treatment reduces parvalbumin expression and perineuronal nets in gamma-aminobutyric acidergic interneurons of the frontal cortex in adult mice. Mol Brain 6:43. 
Pollock E, Everest M, Brown A, Poulter MO (2014) Metalloproteinase inhibition prevents inhibitory synapse reorganization and seizure genesis. Neurobiol Dis 70:21-31.

Puścian A, Winiarski M, Łęski S, Charzewski Ł, Nikolaev T, Borowska J, Dzik JM, Bijata M, Lipp $\mathrm{H}-\mathrm{P}$, Dziembowska M, Knapska E (2021) Chronic fluoxetine treatment impairs motivation and reward learning by affecting neuronal plasticity in the central amygdala. Br J Pharmacol 178:672-688.

Reichelt AC, Hare DJ, Bussey TJ, Saksida LM (2019) Perineuronal Nets: Plasticity, Protection, and Therapeutic Potential. Trends Neurosci 42:458-470.

Schmidt KE, Kuepper JM, Schumak B, Alferink J, Hofmann A, Howland SW, Rénia L, Limmer A, Specht S, Hoerauf A (2018) Doxycycline inhibits experimental cerebral malaria by reducing inflammatory immune reactions and tissue-degrading mediators. PloS One 13:e0192717.

Schreurs BG, Burhans LB, Smith-Bell CA, Mrowka SW, Wang D (2013) Ontogeny of trace eyeblink conditioning to shock-shock pairings in the rat pup. Behav Neurosci 127:114120.

Seeger G, Brauer K, Ha"rtig W, Bru"ckner G (1994) Mapping of perineuronal nets in the rat brain stained by colloidal iron hydroxide histochemistry and lectin cytochemistry. Neuroscience 58:371-388.

Shen HH (2018) Core Concept: Perineuronal nets gain prominence for their role in learning, memory, and plasticity. Proc Natl Acad Sci U S A 115:9813-9815.

Slaker M, Blacktop JM, Sorg BA (2016) Caught in the Net: Perineuronal Nets and Addiction. Neural Plast 2016:e7538208.

Smith-Bell CA, Schreurs BG (2017) Grouping subjects based on conditioning criteria reveals differences in acquisition rates and in strength of conditioning-specific reflex modification. Neurobiol Learn Mem 145:172-180.

Sorg BA, Berretta S, Blacktop JM, Fawcett JW, Kitagawa H, Kwok JCF, Miquel M (2016) Casting a Wide Net: Role of Perineuronal Nets in Neural Plasticity. J Neurosci 36:11459-11468.

Steinzeig A, Cannarozzo C, Castrén E (2019) Fluoxetine-induced plasticity in the visual cortex outlasts the duration of the naturally occurring critical period. Eur J Neurosci 50:36633673.

Testa D, Prochiantz A, Di Nardo AA (2018) Perineuronal nets in brain physiology and disease. Semin Cell Dev Biol.

Tucker R, Binder L, Viereck C, Hemmings B, Matus A (1988) The sequential appearance of lowand high-molecular-weight forms of MAP2 in the developing cerebellum. J Neurosci 8:4503-4512.

Umemori J, Winkel F, Castrén E, Karpova NN (2015) Distinct effects of perinatal exposure to fluoxetine or methylmercury on parvalbumin and perineuronal nets, the markers of critical periods in brain development. Int J Dev Neurosci Off J Int Soc Dev Neurosci 44:55-64.

Wagner M et al. (2020) Loss of TNR causes a nonprogressive neurodevelopmental disorder with spasticity and transient opisthotonus. Genet Med Off J Am Coll Med Genet.

Wang C-T, Zhang L, Wu H-W, Wei L, Xu B, Li D-M (2014) Doxycycline attenuates acute lung injury following cardiopulmonary bypass: involvement of matrix metalloproteinases. Int $\mathrm{J}$ Clin Exp Pathol 7:7460-7468.

Wang D, Darwish DS, Schreurs BG (2006) Effects of 4-aminopyridine on classical conditioning of the rabbit (Oryctolagus cuniculus) nictitating membrane response. Behav Pharmacol 17:319-329.

Wang D, Smith-Bell CA, Burhans LB, O'Dell DE, Bell RW, Schreurs BG (2018) Changes in membrane properties of rat deep cerebellar nuclear projection neurons during acquisition of eyeblink conditioning. Proc Natl Acad Sci 115:E9419-E9428. 
Weber P, Bartsch U, Rasband MN, Czaniera R, Lang Y, Bluethmann H, Margolis RU, Levinson SR, Shrager P, Montag D, Schachner M (1999) Mice Deficient for Tenascin-R Display Alterations of the Extracellular Matrix and Decreased Axonal Conduction Velocities in the CNS. J Neurosci 19:4245-4262.

Xue Y-X, Xue L-F, Liu J-F, He J, Deng J-H, Sun S-C, Han H-B, Luo Y-X, Xu L-Z, Wu P, Lu L (2014) Depletion of Perineuronal Nets in the Amygdala to Enhance the Erasure of Drug Memories. J Neurosci 34:6647-6658.

Yoshioka N, Miyata S, Tamada A, Watanabe Y, Kawasaki A, Kitagawa H, Takao K, Miyakawa T, Takeuchi K, Igarashi M (2017) Abnormalities in perineuronal nets and behavior in mice lacking CSGalNAcT1, a key enzyme in chondroitin sulfate synthesis. Mol Brain $10: 47$. 
Chapter 5. Conclusions 


\subsection{Some Final Queries}

One of the final questions involved rats who were classified as "low learners", $\% \mathrm{CR}<70 \%$ during any day of acquisition. Since the infusion of ChABC or injection of doxycycline could be influencing the rats' ability to obtain $E B C$, it may be worth our while to analyze every animal that had a viable EMG signal-to-noise ratio but but did not reach our learning criterion. We chose to run another analysis of the behavioral statistics after adding "low learners" back in. For the doxycycline group, the only animals that were removed were due to poor signal so their ability to learn EBC could not be determined. However, in the ChABC study, we found seven

A

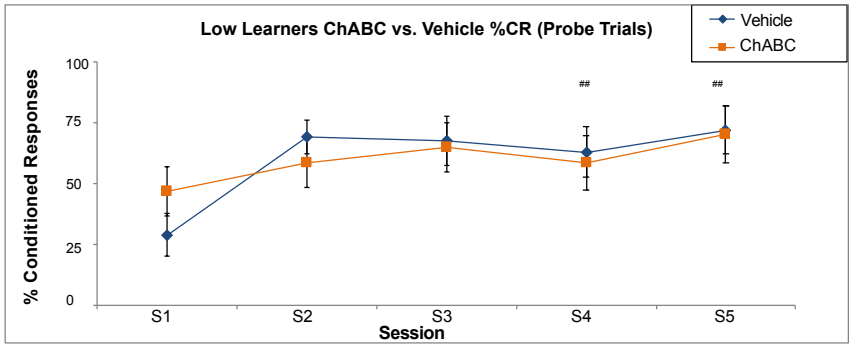

B

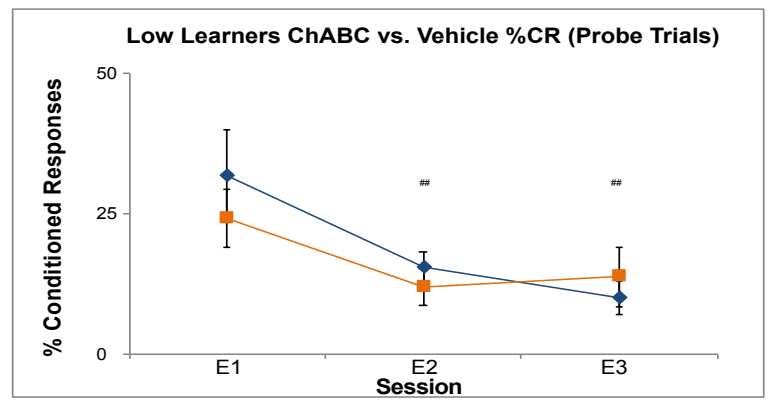

Figure 5.1. Percent Conditioned Responses between low learning ChABC and Vehicle Rats. A. There were no significant differences in \%CRs between the two groups but there were significant sessions differences, showing both groups are capable of learning EBC. B. There were differences between sessions during extinction. \# $=p<.05$ for session

animals that could be included in the statistical analysis. Two of these animals had decent signal but had URs under $20 \%$ as well as CRs under $70 \%$, suggesting they were unable to detect the ES. Five rats were ultimately chosen to be included ( $n=3$ ChABC, $n=2$ vehicle). The parameters of behavior are identical to those found in Chapter 3 other than including all rats as long as they had viable EMG signal and UR > $20 \%$.

\subsubsection{Low Learners}


Although the majority of the relationships remained very similar, the increase in \%CRs for both groups across sessions remained, $[F(4,105)=2.81, p=.029]$, but by including the animals who learned poorly, the overall strength of conditioning was lower and was less powerful statistically. Unsurprisingly, these animals also had session decreases during extinction, $[F(2,58)=7.12, p=.002]$. We did not find any new group differences in \%CR.

The group differences on CR parameters found in the first analysis also persisted. The session differences $[F(4,90)=4.31$, $p=.003]$, and the group differences $[F(1,90)=6.91, p=.010]$ in $C R$ amplitude remained. As did the session differences in the CR area, [F $(4,90)=7.72, p<.000]$, and the A

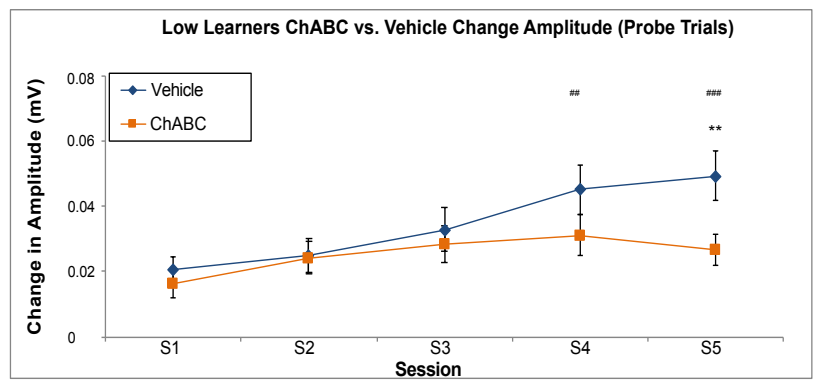

ChABC group still had a smaller $\mathrm{CR}$ area compared to the vehicle group, $[F(1,90)=15.28, p<.000]$. However, the relationship on the first session of $\mathrm{CR}$ area has been eliminated in the new analysis. The group differences appear to resemble amplitude and are driven by smaller responses on the final acquisition sessions. There were some new group differences observed in $\mathrm{CR}$ timing after including the low B

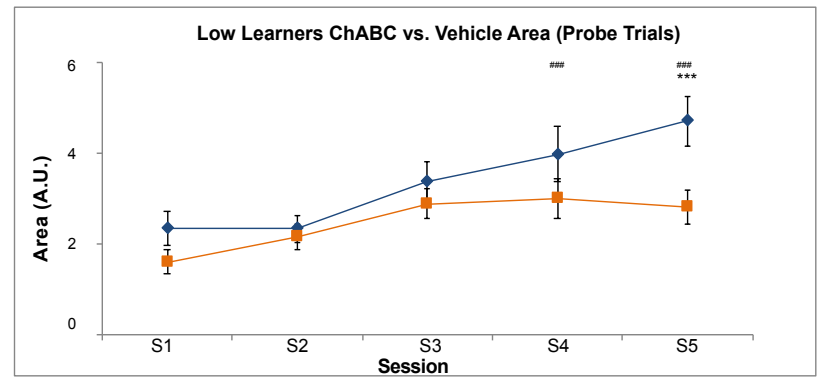

Figure 5.2. Amplitude and Area of the Conditioned Response between low learning ChABC and Vehicle Rats.

A. On S5, the vehicle group had a noticeably higher amplitude on the final acquisition session. B. On S5, the vehicle group had a noticeably higher area under the curve.

There were also significant session differences on S4 and S5 showing the amplitude and area significantly increased over the acquisition period. $\# \#=p<.05, \# \#=p<.000$ for session ${ }^{* *}=p<.01,{ }^{* * *}=p<.000$ for group

learners. The ChABC infused rats had a slower peak eyeblink latency, $[F(1,90)=7.14, p=.009]$, 
compared to control. The session differences in CR latency, $[F(4,90)=3.73, p=.007]$, remained consistent with the initial behavioral assessment, with the response speeding up over sessions.

As before, there were no significant UR differences in any parameters $(F<1)$. Taken together, the new analysis suggests that the ChABC infused rats had a slower eyeblink response as well as the original observation of reduced eyeblink amplitude and area responses. These data reinforce the idea that although our $\mathrm{ChABC}$ infused animals are capable of learning $\mathrm{EBC}$, their associations are not as well stabilized as those with an undigested PNN.

\subsubsection{Low Learners and ChABC digestion levels}

In addition, we also wanted to assess if there were any differences in behavior related to the amount of digestion of the PNN with the low learners included in the analysis. First, the ChABC infused rats were split into WFA+ or WFA-

A

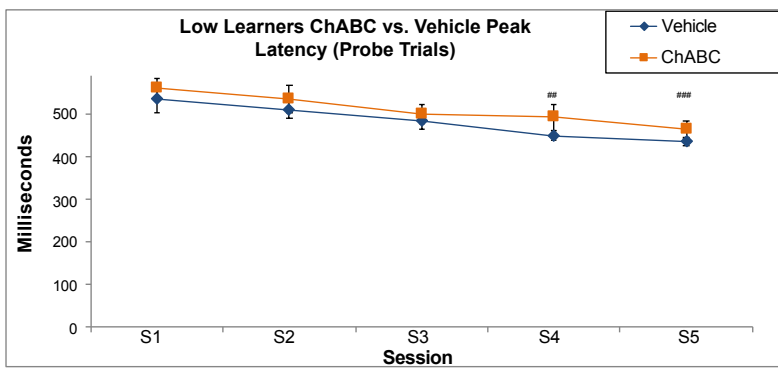
depending on the amount of \%WFA neurons they had. Rats with less than $55 \% \% W F A+$ neurons in the ChABC group, were considered WFA- $(44.7 \pm$ $4.6 \%$ ) and rats above that were considered WFA+ $(65.3 \pm 5.5 \%)$. The vehicle animals had a higher significantly higher percentage of $\mathrm{PNN}+$ neurons, $(86.4 \pm 2.5 \%), p<.000$ for WFA-, and $p=$ .012 for WFA+. WFA- also had a lower B

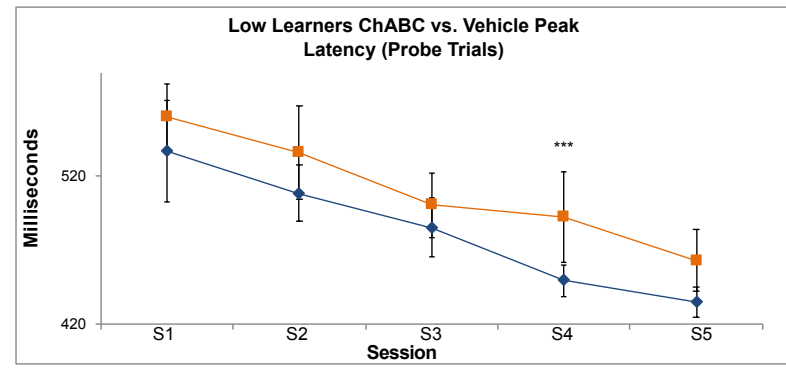

Figure 5.3. Eyeblink Latency of the Conditioned Response between low learning ChABC and Vehicle Rats.

A. The eyeblink response of both groups increased over time. B. There were group differences on S4, with ChABC infused rats having a slower response $\# \#=p<.05$, \#\#\# = p $<.000$ for session $* * *=p<.000$ for group

percentage of $\mathrm{PNN}+$ neurons than WFA+, $p=.028$. We found that ChABC infused rats in the 
WFA- group had significantly lower amplitude $(p=.020)$ and area $(p=.025)$ compared to the WFA+ rats. This suggests that the level of ChABC digestion may play the most critical role in altering behavior.

\subsection{Final Thoughts and Future Directions}

The ChABC experiments yielded a great deal of information related to the PNN in the DCN. The ontogenetic studies we completed were a valuable insight into why EBC may be so difficult to acquire and retain in young animals. The most recent analyses

A

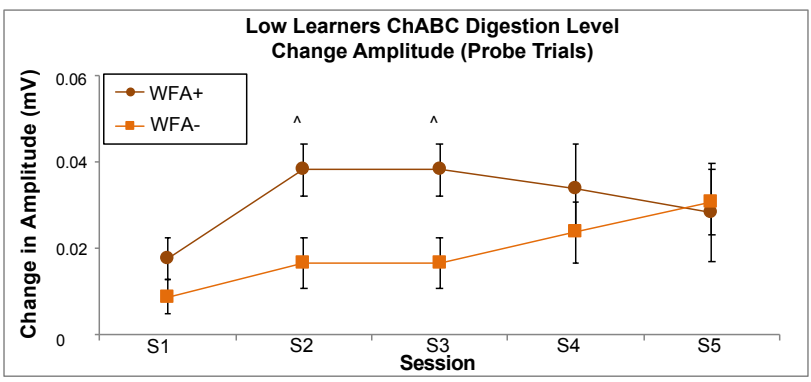
suggest that without adjusting criterion learning to be more inclusive of low learning animals, some relationships may go undetected. However, in spite of eliminating the low learning group, we still see many group differences that indicate $E B C$ is not well stabilized in comparison to the vehicle groups.

This work fits into the larger body of literature by being the third study to examine the relationship between the PNN and EBC in

B

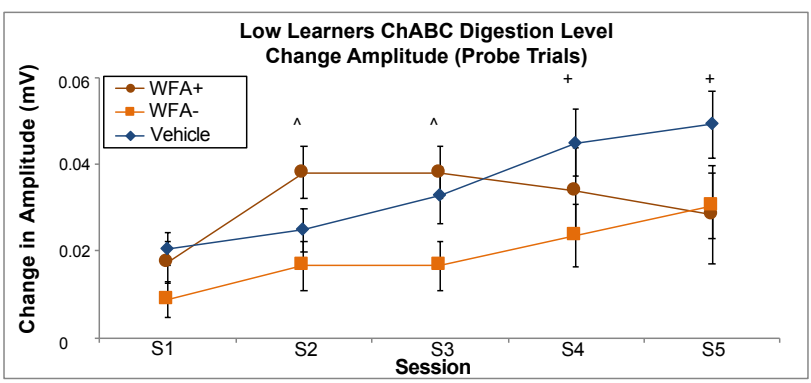

Figure 5.4. Amplitude of the Conditioned Response between low learning ChABC and Vehicle Rats.

A. On S2 and S3, the WFA+ group had a noticeably higher amplitude. B. On S5, the vehicle group has a higher amplitude than either group.

$\Lambda=p<.05$ for WFA differences

$+=p<.05$ for group

rodents and the first to examine these subjects in adult rats (Carulli et al., 2020; Hirono et al., 2018). Our work suggests that even moderate digestion of the PNN can alter behavior. Our in vivo digestion levels were less than the work done by Carulli et a., in 2020 but more robust than the digestion observed in the work done by Hirono et al., in 2018. Perhaps extending our digestion window out to six full days may allow for more animals to reach the level of WFA- animals, who seemed to drive the behavioral differences. In addition, a logical next series of experiments 
would be to examine how exposure to EBC alters the PNN in young animals. Other work has shown that exposing young animals to aversive conditions alters the PNN in other brain regions like the amygdala or hippocampus (Gildawie et al., 2021, 2020; Murthy et al., 2019; Santiago et al., 2018; Yu et al., 2020). Although our lab and collaborators have studied EBC in rats ranging from P12 to P24, there has been no examination of the effects of exposure to the aversive training on the PNN in the DCN. Further, performing ChABC infusion into the DCN of animals under P32 would be another interesting study. This would allow us to study the behavior as well as perform electrophysiology experiments in animals that in vivo ChABC digestion of the PNN. Comparing the intrinsic membrane properties of these animals to the tissue exposed to ChABC in vitro could yield more complete stories on how the PNN functions in this brain region.

We are the first group to compare sex differences in the behavior of rats given EBC and ChABC. Since ChABC eliminated the differences in the vehicle group, we would expect that there could be differences in the PNN of male and female animals. However, whenever these analyses were performed, we did not detect significant differences between male and female animals in either the $\mathrm{ChABC}$ or the vehicle group. We suspect that instead of these differences being directly related to the PNN, they are related to the differences in male and female learning of aversive behavior (Dalla et al., 2009; Dalla and Shors, 2009; Maeng et al., 2010; Maeng and Shors, 2013; Schreurs et al., 2018). Since one of the roles of the PNN is to maintain synaptic stability, the learning differences in the sexes are preserved in the vehicle animals since their PNN remains intact. The ChABC group, likely lost these differences as a result of the PNN no longer cementing these synaptic changes into place. An interesting future experiment could look for specific synaptic changes between the males and females of each group.

The doxycycline experiments have also yielded useful information regarding the future of drug repurposing to manipulate the PNN. By doing an intensive comparison of the shock intensity and tone intensity, we would be able to determine what precisely is being sensitized by this antibiotic (Wang et al., 2006). Unpaired controls would also be a beneficial group to add into the 
study (Campolattaro and Freeman, 2009; Goldsberry et al., 2014; McEchron et al., 2003; Schreurs et al., 1998). Another option to consider would be systemic administration of doxycycline via oral gavage or by providing it in drinking water. If this route of manipulating the PNN seems to truly alter the UR, an alternative would be to use the antidepressant fluoxetine. This drug appears to have had more success in behavioral studies in mice, rats, and rabbits (Burhans et al., 2013; Deschaux et al., 2013; Karpova et al., 2011; Kiryanova et al., 2017; Lesnikova et al., 2021; Ohira et al., 2013; Pawluski et al., 2014; Steinzeig et al., 2019; Umemori et al., 2018, 2015). In addition to confirming UR differences, investigation of changes in the PNN throughout the brain following systemic doxycycline should be done. In our initial pilot studies of acute doxycycline injections, we noticed there was a marginal difference between the PNN in the amygdala of the rats given doxycycline compared to vehicle. These differences in other brain regions may also be useful studies to help us gain information on how doxycycline is altering the PNN.

Another crucial series of experiments that must be performed with the doxycycline studies would be to perform gelatin zymography experiments to analyze the actual activity of MMP9. We do not know for certain if our drugs were actually successful at inhibiting MMP9 and how much inhibition, if any, was occurring during behavior. This could allow us to determine the best dosage for MMP9 inhibition. Perhaps our initial dosage of $40 \mathrm{mg} / \mathrm{kg}$ is inappropriate by either being far too high or far too low. Increasing the dosage should be taken with great care, since higher doses in rats can prove to be fatal. Did we also begin doxycycline administration too closely to behavior? It may be necessary to start giving the rats doxycycline a week or so prior to behavior even begins. Additionally, there are other drugs that can are more potent MMP9 inhibitors we could compare to doxycycline.

In addition to performing the zymography with animals in the doxycycline study, it would be intriguing to know if exposure to EBC itself could alter the activity of this enzyme. We could perform EMG surgeries in all rats but only expose a subset of rats to EBC while the others act as 
sit controls. These animals are never presented with the CS or the US but simply sit in the chamber for the duration of the sessions. Since we noted that there may be a significant reduction in the PNN of trained animals compared to completely naïve rats it would be useful to know that this is the result of increased MMP9 activity. Additionally, does time after learning alter PNN digestion levels? Carulli et al., noted that the level of digestion returned to pre-learning levels by the end of all behavior (Carulli et al., 2020). What if compared the PNN to animals euthanized only an hour or so after the final acquisition session? Would we see a greater reduction in the PNN and increased activity of MMP9 as a result?

Although we make the assumption that all of our observations are the direct result of PNN manipulation, we cannot rule out other unknown factors that may be playing a role. $\mathrm{ChABC}$ is only a temporary digestion of the PNN. However, the CSPG A that is removed from the PNN remains present in the extracellular space months even after the nets have been reassembled (Brückner et al., 1998). No one has examined how ChABC infusion could impact behavior long-term after the PNN has recovered either. Based on our own and others' electrophysiological recordings, ChABC does not appear to harm neurons but its effects on

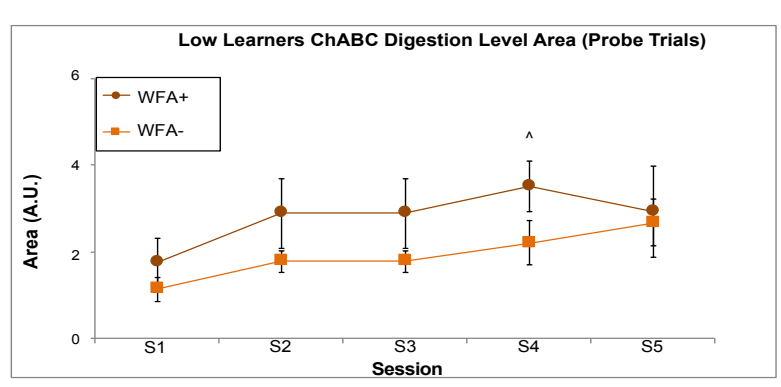

B

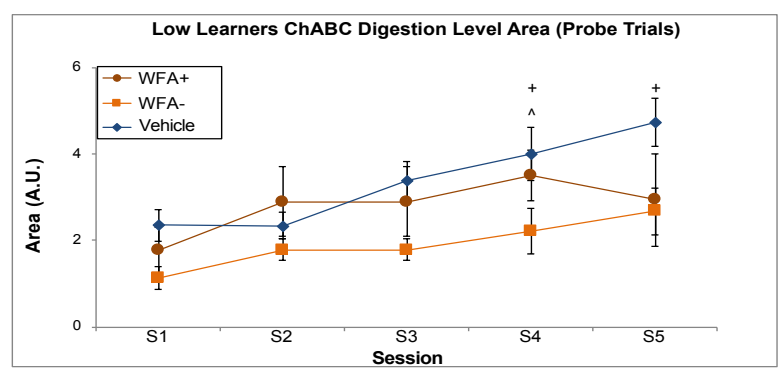

Figure 5.5. Area of the Conditioned Response between low learning $C h A B C$ and Vehicle Rats.

A. On S4, the WFA+ group had a higher area. B. On S4 and $\mathrm{S} 5$, the vehicle group has a higher amplitude than either group.

$\wedge=p<.05$ for WFA differences

$+=p<.05$ for group

glia or the vasculature are not well known (Cope and Gould, 2019; Stoyanov et al., 2021; Wegrzyn et al., 2021). Using markers for glial activation could be useful to further determine if ChABC causes any inflammatory effects 
Another potential experiment would be to compare the results between the behavioral data of rats given $\mathrm{ChABC}$ infusion and those given an infusion of hyaluronidase (Bilong et al., 2021; Greda and Nowicka, 2020; Iwata et al., 1993; Murase et al., 2017). This enzyme would target the hyaluronic acid backbone of the PNN. The vast majority of PNN work uses ChABC but there is a subset using this alternative enzyme. We would expect the results to be similar following hyaluronidase infusion, but no one has examined how this enzyme alters the PNN and EBC in rodents at this point.

Although they are highly informative, transgenic studies have imperfections due to animals developing without parts of the PNN present. Animals that grow up without a PNN component may have some method of compensating for that loss. The inducible transgenic models I thought of in Chapter 1 may have confounding factors after continuing to delve into the literature. Doxycycline is one option to induce a transgenic mutation (Belteki et al., 2005; Chow et al., 2012; Matsushita et al., 2013; Saunders, 2011), but as we have demonstrated, there could be direct manipulation of the PNN. Additionally others have noticed that doxycycline could be influencing cell or animal behavior independently of the desired transgenic effects (Cao et al., 2019; Mclver et al., 2012). Tamoxifen is another commonly used drug to induce transgenic mutations (Feil et al., 2009; Leone et al., 2003; Sassmann et al., 2010). However, this drug influences estrogen receptors (Shagufta and Ahmad, 2018), which could then modulate any PNN related sex differences (Drzewiecki et al., 2020; Gildawie et al., 2021, 2020; Guadagno et al., 2020). However, by careful study and combining several of these methods, the PNNs influence can hopefully be better understood. For example, some researchers have tried to determine the strain and region-specific differences in how tamoxifen is processed in the mouse brain (Valny et al., 2016), which could be used to potentially allow the mice to return to normal estrogen function while the PNN is degraded before further testing is done. By combining these techniques, it should be possible to determine the full function of the PNN. 
This research contributes greatly to the field. Firstly, it is one of the few research projects investigating the minority of PNN+ excitatory cells. Although understanding the role of the PNN on inhibitory cells is crucial, we cannot ignore the smaller population of excitatory neurons or we will never truly understand the function of the net. Secondly, our work here is some of the only work focusing on cerebellar dependent learning and the only published work examining the effect of the PNN in EBC in rats. By continuing this work in other animals that have well characterized EBC, like rabbits for example, we will understand how the PNN functions in various brain regions and processes. Thirdly, this work strongly suggests that even moderate digestion of the PNN can alter behavior and learning and memory processes. We observed decreased excitability of neurons in the DCN, which is negatively correlated to high levels of associative learning (Moyer et al., 2000, 1996; Oh and Disterhoft, 2015; Thompson et al., 1996). This could be relevant when investigating diseases that seem to affect the PNN or PNN+ neurons. Even minor changes to the PNN could be influencing the activity of neurons and behavior. Fourthly, the sex differences we observed in our vehicle animals suggests that even though the PNN itself may be similar between male and female animals, it could play a role in the learning or synaptic differences that are sex dependent. Lastly, this work could provide data that indicates using doxycycline in behavior or transgenic studies should be handled with great care due to unexpected influence on the PNN or on non-associative effects. Taken together, this project provided more insight into how the PNN can play a role in learning and memory processes and how attempting to translate it into a therapeutic target can be achieved successfully in the future. 


\section{References:}

Belteki, G., Haigh, J., Kabacs, N., Haigh, K., Sison, K., Costantini, F., Whitsett, J., Quaggin, S.E., Nagy, A., 2005. Conditional and inducible transgene expression in mice through the combinatorial use of Cre-mediated recombination and tetracycline induction. Nucleic Acids Res. 33, e51. https://doi.org/10.1093/nar/gni051

Bilong, M., Bayat, P., Bourderioux, M., Jérôme, M., Giuliani, A., Daniel, R., 2021. Mammal Hyaluronidase Activity on Chondroitin Sulfate and Dermatan Sulfate: Mass Spectrometry Analysis of Oligosaccharide Products. Glycobiology. https://doi.org/10.1093/glycob/cwab004

Brückner, G., Bringmann, A., Härtig, W., Köppe, G., Delpech, B., Brauer, K., 1998. Acute and long-lasting changes in extracellular-matrix chondroitin-sulphate proteoglycans induced by injection of chondroitinase ABC in the adult rat brain. Exp. Brain Res. 121, 300-310. https://doi.org/10.1007/s002210050463

Burhans, L.B., Smith-Bell, C.A., Schreurs, B.G., 2013. Subacute fluoxetine enhances conditioned responding and conditioning-specific reflex modification of the rabbit nictitating membrane response: implications for drug treatment with selective serotonin reuptake inhibitors. Behav. Pharmacol. 24, 55-64. https://doi.org/10.1097/FBP.0b013e32835d528e

Campolattaro, M.M., Freeman, J.H., 2009. An Examination of Bilateral Eyeblink Conditioning in Rats. Behav. Neurosci. 123, 1346-1352. https://doi.org/10.1037/a0017314

Cao, D., Cheung, H.-H., Chan, W.-Y., 2019. Doxycycline Masks the Genuine Effect of the Doxycycline-Inducible Transgene by Promoting Dopaminergic Neuron Differentiation from Human Pluripotent Stem Cells. Stem Cells Dev. 28, 833-845. https://doi.org/10.1089/scd.2018.0209

Carulli, D., Broersen, R., de Winter, F., Muir, E.M., Mešković, M., de Waal, M., de Vries, S., Boele, H.-J., Canto, C.B., De Zeeuw, C.I., Verhaagen, J., 2020. Cerebellar plasticity and associative memories are controlled by perineuronal nets. Proc. Natl. Acad. Sci. U. S. A. https://doi.org/10.1073/pnas.1916163117

Chow, J.D.Y., Price, J.T., Bills, M.M., Simpson, E.R., Boon, W.C., 2012. A doxycyclineinducible, tissue-specific aromatase-expressing transgenic mouse. Transgenic Res. 21, 415-428. https://doi.org/10.1007/s11248-011-9525-7

Cope, E.C., Gould, E., 2019. Adult Neurogenesis, Glia, and the Extracellular Matrix. Cell Stem Cell 24, 690-705. https://doi.org/10.1016/j.stem.2019.03.023

Dalla, C., Papachristos, E.B., Whetstone, A.S., Shors, T.J., 2009. Female rats learn trace memories better than male rats and consequently retain a greater proportion of new neurons in their hippocampi. Proc. Natl. Acad. Sci. U. S. A. 106, 2927-2932. https://doi.org/10.1073/pnas.0809650106

Dalla, C., Shors, T.J., 2009. Sex differences in learning processes of classical and operant conditioning. Physiol. Behav., Sex differences in physiology and behavior: focus on central actions of ovarian hormones 97, 229-238. https://doi.org/10.1016/j.physbeh.2009.02.035

Deschaux, O., Zheng, X., Lavigne, J., Nachon, O., Cleren, C., Moreau, J.-L., Garcia, R., 2013. Post-extinction fluoxetine treatment prevents stress-induced reemergence of extinguished fear. Psychopharmacology (Berl.) 225, 209-216. https://doi.org/10.1007/s00213-012-2806-x

Drzewiecki, C.M., Willing, J., Juraska, J.M., 2020. Influences of age and pubertal status on number and intensity of perineuronal nets in the rat medial prefrontal cortex. Brain Struct. Funct. https://doi.org/10.1007/s00429-020-02137-z

Feil, S., Valtcheva, N., Feil, R., 2009. Inducible Cre mice. Methods Mol. Biol. Clifton NJ 530, 343-363. https://doi.org/10.1007/978-1-59745-471-1_18 
Gildawie, K.R., Honeycutt, J.A., Brenhouse, H.C., 2020. Region-specific Effects of Maternal Separation on Perineuronal Net and Parvalbumin-expressing Interneuron Formation in Male and Female Rats. Neuroscience 428, 23-37. https://doi.org/10.1016/j.neuroscience.2019.12.010

Gildawie, K.R., Ryll, L.M., Hexter, J.C., Peterzell, S., Valentine, A.A., Brenhouse, H.C., 2021. A two-hit adversity model in developing rats reveals sex-specific impacts on prefrontal cortex structure and behavior. Dev. Cogn. Neurosci. 48, 100924. https://doi.org/10.1016/j.dcn.2021.100924

Goldsberry, M.E., Elkin, M.E., Freeman, J.H., 2014. Sensory system development influences the ontogeny of eyeblink conditioning. Dev. Psychobiol. 56, 1244-1251. https://doi.org/10.1002/dev.21204

Greda, A.K., Nowicka, D., 2020. Hyaluronidase inhibition accelerates functional recovery from stroke in the mouse brain. J. Neurochem. n/a. https://doi.org/10.1111/jnc.15279

Guadagno, A., Verlezza, S., Long, H., Wong, T.P., Walker, C.-D., 2020. It is all in the right amygdala: increased synaptic plasticity and perineuronal nets in male, but not female juvenile rat pups after exposure to early-life stress. J. Neurosci. https://doi.org/10.1523/JNEUROSCI.1029-20.2020

Hirono, M., Watanabe, S., Karube, F., Fujiyama, F., Kawahara, S., Nagao, S., Yanagawa, Y., Misonou, H., 2018. Perineuronal nets in the deep cerebellar nuclei regulate GABAergic transmission and delay eyeblink conditioning. J. Neurosci. 3238-17. https://doi.org/10.1523/JNEUROSCI.3238-17.2018

Iwata, M., Wight, T.N., Carlson, S.S., 1993. A brain extracellular matrix proteoglycan forms aggregates with hyaluronan. J. Biol. Chem. 268, 15061-15069.

Karpova, N.N., Pickenhagen, A., Lindholm, J., Tiraboschi, E., Kulesskaya, N., Agústsdóttir, A., Antila, H., Popova, D., Akamine, Y., Bahi, A., Sullivan, R., Hen, R., Drew, L.J., Castrén, E., 2011. Fear erasure in mice requires synergy between antidepressant drugs and extinction training. Science 334, 1731-1734. https://doi.org/10.1126/science.1214592

Kiryanova, V., Meunier, S.J., Dyck, R.H., 2017. Behavioural outcomes of adult female offspring following maternal stress and perinatal fluoxetine exposure. Behav. Brain Res. 331, 8491. https://doi.org/10.1016/j.bbr.2017.05.029

Leone, D.P., Genoud, S., Atanasoski, S., Grausenburger, R., Berger, P., Metzger, D., Macklin, W.B., Chambon, P., Suter, U., 2003. Tamoxifen-inducible glia-specific Cre mice for somatic mutagenesis in oligodendrocytes and Schwann cells. Mol. Cell. Neurosci. 22, 430-440. https://doi.org/10.1016/s1044-7431(03)00029-0

Lesnikova, A., Casarotto, P.C., Fred, S.M., Voipio, M., Winkel, F., Steinzeig, A., Antila, H., Umemori, J., Biojone, C., Castrén, E., 2021. Chondroitinase and Antidepressants Promote Plasticity by Releasing TRKB from Dephosphorylating Control of PTPo in Parvalbumin Neurons. J. Neurosci. 41, 972-980. https://doi.org/10.1523/JNEUROSCI.2228-20.2020

Maeng, L.Y., Shors, T.J., 2013. The stressed female brain: neuronal activity in the prelimbic but not infralimbic region of the medial prefrontal cortex suppresses learning after acute stress. Front. Neural Circuits 7. https://doi.org/10.3389/fncir.2013.00198

Maeng, L.Y., Waddell, J., Shors, T.J., 2010. The Prefrontal Cortex Communicates with the Amygdala to Impair Learning after Acute Stress in Females but Not in Males. J. Neurosci. 30, 16188-16196. https://doi.org/10.1523/JNEUROSCI.2265-10.2010

Matsushita, N., Matsushita, S., Hirakawa, S., Higashiyama, S., 2013. Doxycycline-dependent inducible and reversible RNA interference mediated by a single lentivirus vector. Biosci. Biotechnol. Biochem. 77, 776-781. https://doi.org/10.1271/bbb.120917

McEchron, M.D., Tseng, W., Disterhoft, J.F., 2003. Single Neurons in CA1 Hippocampus Encode Trace Interval Duration during Trace Heart Rate (Fear) Conditioning in Rabbit. J. Neurosci. 23, 1535-1547. https://doi.org/10.1523/JNEUROSCl.23-04-01535.2003 
Mclver, S.R., Muccigrosso, M.M., Haydon, P.G., 2012. The effect of doxycycline on alcohol consumption and sensitivity: consideration for inducible transgenic mouse models. Exp. Biol. Med. Maywood NJ 237, 1129-1133. https://doi.org/10.1258/ebm.2012.012029

Moyer, J.R., Power, J.M., Thompson, L.T., Disterhoft, J.F., 2000. Increased excitability of aged rabbit CA1 neurons after trace eyeblink conditioning. J. Neurosci. Off. J. Soc. Neurosci. 20, 5476-5482.

Moyer, J.R., Thompson, L.T., Disterhoft, J.F., 1996. Trace eyeblink conditioning increases CA1 excitability in a transient and learning-specific manner. J. Neurosci. Off. J. Soc. Neurosci. 16, 5536-5546.

Murase, S., Lantz, C., Quinlan, E., 2017. Light reintroduction after dark exposure reactivates plasticity in adults via perisynaptic activation of MMP-9. eLife 6. https://doi.org/10.7554/eLife.27345

Murthy, S., Kane, G.A., Katchur, N.J., Lara Mejia, P.S., Obiofuma, G., Buschman, T.J., McEwen, B.S., Gould, E., 2019. Perineuronal Nets, Inhibitory Interneurons, and AnxietyRelated Ventral Hippocampal Neuronal Oscillations Are Altered by Early Life Adversity. Biol. Psychiatry, Adversity and Posttraumatic Stress Disorder 85, 1011-1020. https://doi.org/10.1016/j.biopsych.2019.02.021

Oh, M.M., Disterhoft, J.F., 2015. Increased Excitability of Both Principal Neurons and Interneurons during Associative Learning: The Neuroscientist. https://doi.org/10.1177/1073858414537382

Ohira, K., Takeuchi, R., Iwanaga, T., Miyakawa, T., 2013. Chronic fluoxetine treatment reduces parvalbumin expression and perineuronal nets in gamma-aminobutyric acidergic interneurons of the frontal cortex in adult mice. Mol. Brain 6, 43. https://doi.org/10.1186/1756-6606-6-43

Pawluski, J.L., van Donkelaar, E., Abrams, Z., Houbart, V., Fillet, M., Steinbusch, H.W.M., Charlier, T.D., 2014. Fluoxetine Dose and Administration Method Differentially Affect Hippocampal Plasticity in Adult Female Rats [WWW Document]. Neural Plast. https://doi.org/10.1155/2014/123026

Santiago, A.N., Lim, K.Y., Opendak, M., Sullivan, R.M., Aoki, C., 2018. Early life trauma increases threat response of peri-weaning rats, reduction of axo-somatic synapses formed by parvalbumin cells and perineuronal net in the basolateral nucleus of amygdala. J. Comp. Neurol. 0. https://doi.org/10.1002/cne.24522

Sassmann, A., Offermanns, S., Wettschureck, N., 2010. Tamoxifen-inducible Cre-mediated recombination in adipocytes. Genes. N. Y. N 2000 48, 618-625. https://doi.org/10.1002/dvg.20665

Saunders, T.L., 2011. Inducible transgenic mouse models. Methods Mol. Biol. Clifton NJ 693, 103-115. https://doi.org/10.1007/978-1-60761-974-1_7

Schreurs, B.G., Gusev, P.A., Tomsic, D., Alkon, D.L., Shi, T., 1998. Intracellular correlates of acquisition and long-term memory of classical conditioning in Purkinje cell dendrites in slices of rabbit cerebellar lobule HVI. J. Neurosci. Off. J. Soc. Neurosci. 18, 5498-5507.

Schreurs, B.G., Smith-Bell, C., Burhans, L.B., 2018. Sex differences in a rabbit eyeblink conditioning model of PTSD. Neurobiol. Learn. Mem. 155, 519-527. https://doi.org/10.1016/j.nIm.2018.04.015

Shagufta, null, Ahmad, I., 2018. Tamoxifen a pioneering drug: An update on the therapeutic potential of tamoxifen derivatives. Eur. J. Med. Chem. 143, 515-531. https://doi.org/10.1016/j.ejmech.2017.11.056

Steinzeig, A., Cannarozzo, C., Castrén, E., 2019. Fluoxetine-induced plasticity in the visual cortex outlasts the duration of the naturally occurring critical period. Eur. J. Neurosci. 50, 3663-3673. https://doi.org/10.1111/ejn.14512

Stoyanov, S., Sun, W., Düsedau, H.P., Cangalaya, C., Choi, I., Mirzapourdelavar, H., BaidoeAnsah, D., Kaushik, R., Neumann, J., Dunay, I.R., Dityatev, A., 2021. Attenuation of the 
extracellular matrix restores microglial activity during the early stage of amyloidosis. Glia 69, 182-200. https://doi.org/10.1002/glia.23894

Thompson, L.T., Moyer, J.R., Disterhoft, J.F., 1996. Transient changes in excitability of rabbit CA3 neurons with a time course appropriate to support memory consolidation. J. Neurophysiol. 76, 1836-1849.

Umemori, J., Winkel, F., Castrén, E., Karpova, N.N., 2015. Distinct effects of perinatal exposure to fluoxetine or methylmercury on parvalbumin and perineuronal nets, the markers of critical periods in brain development. Int. J. Dev. Neurosci. Off. J. Int. Soc. Dev. Neurosci. 44, 55-64. https://doi.org/10.1016/j.ijdevneu.2015.05.006

Umemori, J., Winkel, F., Didio, G., Llach Pou, M., Castrén, E., 2018. iPlasticity: induced juvenile-like plasticity in the adult brain as a mechanism of antidepressants. Psychiatry Clin. Neurosci. https://doi.org/10.1111/pcn.12683

Valny, M., Honsa, P., Kirdajova, D., Kamenik, Z., Anderova, M., 2016. Tamoxifen in the Mouse Brain: Implications for Fate-Mapping Studies Using the Tamoxifen-Inducible Cre-loxP System. Front. Cell. Neurosci. 10, 243. https://doi.org/10.3389/fncel.2016.00243

Wang, D., Darwish, D.S., Schreurs, B.G., 2006. Effects of 4-aminopyridine on classical conditioning of the rabbit (Oryctolagus cuniculus) nictitating membrane response. Behav. Pharmacol. 17, 319-329. https://doi.org/10.1097/01.fbp.0000224381.56121.15

Wegrzyn, D., Freund, N., Faissner, A., Juckel, G., 2021. Poly I:C Activated Microglia Disrupt Perineuronal Nets and Modulate Synaptic Balance in Primary Hippocampal Neurons in vitro. Front. Synaptic Neurosci. 13, 637549. https://doi.org/10.3389/fnsyn.2021.637549

Yu, Z., Chen, N., Hu, D., Chen, W., Yuan, Y., Meng, S., Zhang, W., Lu, L., Han, Y., Shi, J., 2020. Decreased Density of Perineuronal Net in Prelimbic Cortex Is Linked to Depressive-Like Behavior in Young-Aged Rats. Front. Mol. Neurosci. 13, 4. https://doi.org/10.3389/fnmol.2020.00004 